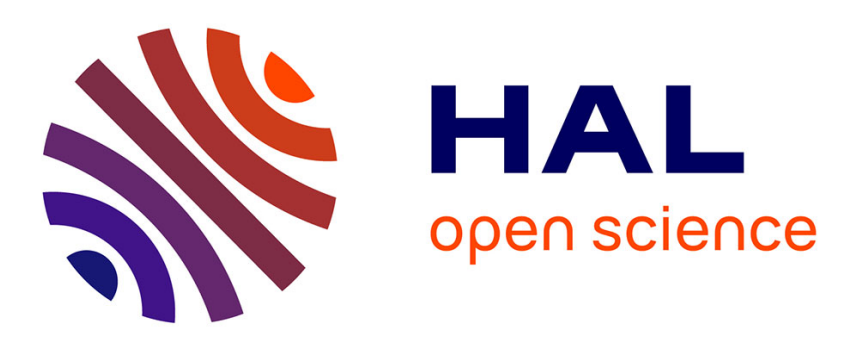

\title{
Modification of the $\beta$-adrenoceptor stimulation pathway in Zucker obese and obese diabetic rat myocardium
}

Cheng Jiang

\section{To cite this version:}

Cheng Jiang. Modification of the $\beta$-adrenoceptor stimulation pathway in Zucker obese and obese diabetic rat myocardium. Tissues and Organs [q-bio.TO]. Université Pierre et Marie Curie - Paris VI; Université de Wuhan (Chine), 2015. English. NNT : 2015PA066726 . tel-01418221

\section{HAL Id: tel-01418221 \\ https://theses.hal.science/tel-01418221}

Submitted on 16 Dec 2016

HAL is a multi-disciplinary open access archive for the deposit and dissemination of scientific research documents, whether they are published or not. The documents may come from teaching and research institutions in France or abroad, or from public or private research centers.
L'archive ouverte pluridisciplinaire HAL, est destinée au dépôt et à la diffusion de documents scientifiques de niveau recherche, publiés ou non, émanant des établissements d'enseignement et de recherche français ou étrangers, des laboratoires publics ou privés. 


\title{
Université Pierre et Marie Curie
}

\author{
Université de Wuhan
}

Ecole Doctorale 394

UMR INSERM-UPMC 1166/Physiologie Physiopathologie et Thérapeutique

\section{Modification de la voie de la stimulation des récepteurs $\beta$-adrénergiques dans le myocarde des rats Zucker obèses et obèses diabétiques}

\author{
Par Cheng JIANG
}

Thèse de doctorat de Physiologie et Physiopathologie

Dirigée par Professeur Bruno RIOU (Université Pierre et Marie Curie, Paris, Frnace) et Professeur Yan ZHAO (Université de Wuhan)

Présentée et soutenue publiquement le 26 juin 2015

Devant un jury composé de :

Mr. le Professeur Shu-sheng LI, Président de thèse

Mr. le Professeur Jean-Emmanuel de LA COUSSAYE, Rapporteur

Mr. le Professeur Li-Bo CHEN, Rapporteur

Mr. le Professeur Bruno RIOU, Examinateur

Mr. le Professeur Yan ZHAO, Examinateur

Mr. le Professeur Julien AMOUR, Examinateur 


\section{Remerciements}

To four outstanding teachers: Professor Bruno Riou, Professor Yan Zhao, Professor Julien Amour and Doctor Aude Carillion, for their trust, support and wise advice which enabled me to carry our this research.

To my rapporteurs, Professeur Jean-Emmanuel de LA COUSSAYE and Professeur Li-Bo CHEN who made me the honor to report my work.

To my important colleagues and friends in France: Ms. Na Na, Ms. Sarah Feldman, Ms. Beibei Wang, and Mr. Qiuji Wu.

To my colleagues and friends in China: Ms. Shan Jiang, Mr. Xianlong Zhou, and all the staffs in the Emergency Center of Zhongnan Hospital of Wuhan University. Thanks a lot for language review by Ms. Wenting Zhou.

To my family.

I want to especially thank the China Scholarship Council (CSC) for the State Scholarship Fund (File NO. 201206270014), the International Office in Wuhan University, and the International Relation Office in Université Pierre et Marie Curie - UPMC. Without their supports and help, I would not have the chance to study and make my research with those leader teams in France. 


\section{Sommaire}

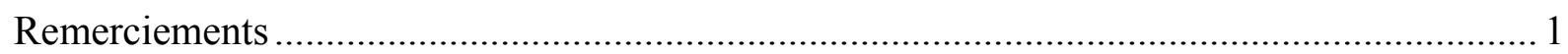

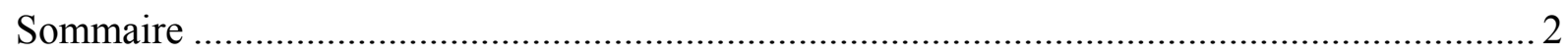

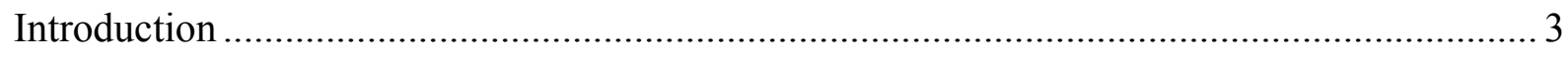

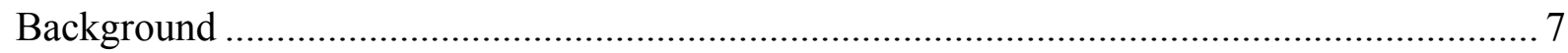

Physiological and pathophysiological $\beta$-adrenergic receptor signaling pathway

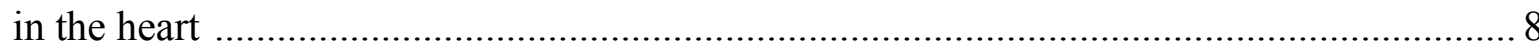

Obesity and diabetes mellitus related cardiomyopathy and the alterations in the

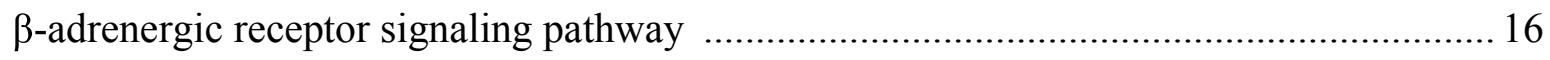

Animal models of obesity, diabetes mellitus and metabolic syndrome ........................... 27

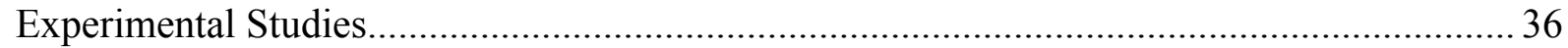

Study No. 1 Over-expression of cyclic adenosine monophosphate effluent protein MRP4 induces an altered response to $\beta$-adrenergic stimulation in the senescent rat heart 37

Study No. 2 Modification of the $\beta$-adrenoceptor stimulation pathway in Zucker obese and

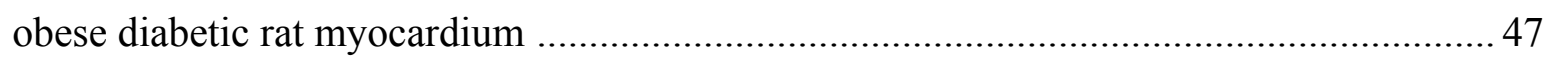

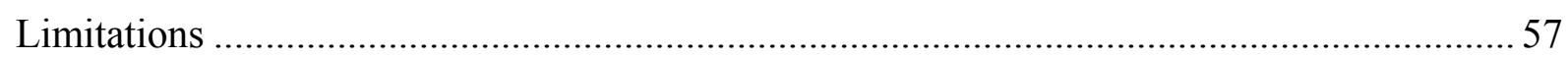

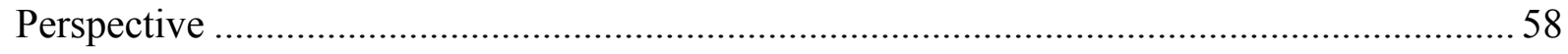

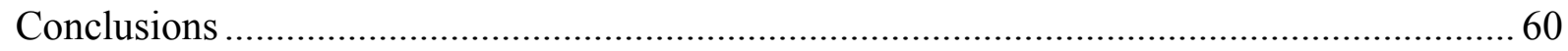

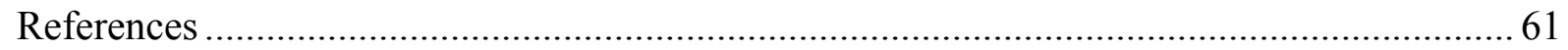

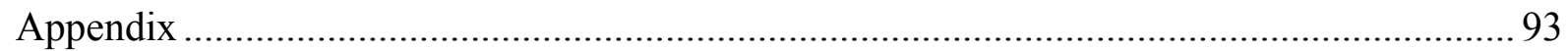

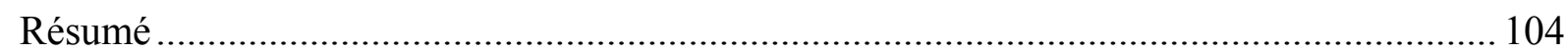

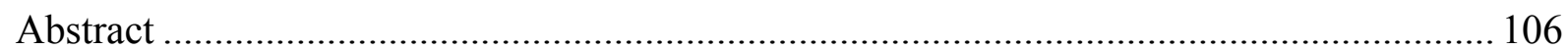

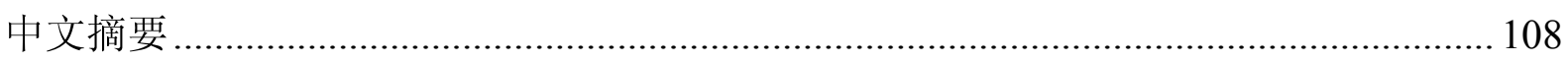




\section{Chapter One}

\section{Introduction}

The prevalence of obesity around the world leads to a remarkable increase in the metabolic syndrome and the metabolic related disorders, including insulin resistance, hyperglycemia, dyslipidemia and hypertension ${ }^{[1,2]}$. The risks of diabetes mellitus and the cardiovascular abnormalities associated with aforementioned factors are also increased. Patients of metabolic syndrome have an about five-fold increase in the risk of diabetes mellitus ${ }^{[3,4]}$. Approximate 1 adult in 4 or 5 has metabolic syndrome, depending on different countries. In the United States of America, approximately 47 million (24\%) adult suffered from the metabolic syndrome in $2002^{[5]}$. The morbidity is increasing in both developed and developing countries ${ }^{[5,6]}$. With the change of diet habits and physical exercise levels in modern life, together with the increasing morbidity of obesity at an early age, the World Health Organization (WHO) projections in 2005 have alarmed for obesity and obesity related diseases, and regarded obesity as a global public health problem ${ }^{[2]}$. Estimated by WHO, in 2015, approximately 2.3 billion adults are overweight and among them, at least 700 million are obese (body mass index (BMI) $>30$ $\left.\mathrm{g} / \mathrm{m}^{2}\right)^{[7]}$. Considering diabetes mellitus, WHO has stated that up to 347 million people worldwide suffered from diabetes mellitus in 2008, and the incidence is so rapidly increasing that estimating number will almost double by $2030^{[8]}$.

Obesity has been regarded as an independent predictor of left ventricular (LV) diastolic dysfunction and can cause depression of cardiac function ${ }^{[9,10]}$. Central obesity is one independent risk factor for cardiovascular disease and is associated with metabolic syndrome ${ }^{[11]}$. Obesity increases the risk of heart failure about two folds even when hypertension and other related risk factors are corrected ${ }^{[12]}$. However, after the onset of heart failure, obesity will be a positive predictor for survival, which is considered as "obesity paradox" ${ }^{[13-15]}$. This phenomenon has also been observed in the perivascular and epicardial white adipose tissues, which demonstrates a potential cardio - protective effects ${ }^{[15]}$. Diabetes mellitus also increases the incidence of heart failure, despite correcting age, hypertension, obesity, hypercholesterolemia and coronary artery disease ${ }^{[16]}$. The diabetic cardiomyopathy is characterized as diastolic dysfunction followed by the systolic dysfunction ${ }^{[17]}$. From the echocardiography of the diabetic patients without known cardiac disease, the damage of early diastolic filling, prolonged isovolumic relaxation and increased atrial filling were observed ${ }^{[18]}$. 
Metabolic syndrome, also as a major risk factor of cardiovascular disease, has been considered as a direct precursor of diabetes mellitus ${ }^{[19-23]}$. The National Cholesterol Education Program's Adult Treatment Panel III (ATPIII) report has identified six components of metabolic syndrome that related to cardiovascular disease, including abdominal obesity, atherogenic dyslipidemia, hypertension, insulin resistance / glucose intolerance, and proinflammatory and prothrombotic states ${ }^{[24]}$.

Pathophysiologically, the onset and progression of obesity, diabetes mellitus and metabolic syndrome are complicated and still not fully elucidated, among which the activation of sympathetic nervous system and a marked adrenergic hyperactivity are mostly observed ${ }^{[2,25-}$ ${ }^{32]}$. Metabolic syndrome is associated with sympathetic activation, possibly attributed to the neural mechanism (direct activation of sympathetic nerve system and renal afferent nerve activation), genetic factors ( $\beta$-adrenoceptor polymorphisms), metabolic factors (hyperinsulinemia, insulin resistance and dysregulated production and secretion of adipokines), reflex factors (an impairment to restrain the adrenergic cardiovascular drive in the baroreflex), psychological stress, oxidative stress, obstructive sleep apnea and inflammation ${ }^{[32,33]}$, among which insulin resistance (IR) is proposed as a "driving force" of the metabolic syndrome ${ }^{[34,35]}$. In the obese normotensive individuals, elevation of sympathetic outflow was observed from the examinations of circulatory catecholamines, urine norepinephrine, muscle sympathetic nerve activity (MSNA) of postganglionic sympathetic nerve fibers, and renal norepinephrine spillover ${ }^{[36,37]}$. In obesity, the degree of the sympathetic activation paralleled closely to the increase of body mass index (BMI ${ }^{[31]}$. Most studies have demonstrated that the $\beta$-adrenoceptor polymorphisms accompanying the sympathetic hyperactivity are associated with hypertension, obesity and diabetes mellitus ${ }^{[38,39]}$. In addition, the sympathetic overdrive can be observed in obese individuals predisposed to metabolic syndrome before hypertension happens ${ }^{[32]}$. And there is also an evidence that obesity per se did not generalize sympathetic hyperactivity, but caused differential activation of tissues sympathetic activities ${ }^{[40,41]}$. The importance of the sympathetic nervous system in the cardiovascular disease has already been clarified from the cardiac arrhythmias, hypertension, cardiomyopathy and progressive heart failure to the final death ${ }^{[2,42]}$.

Previous research about obesity-related cardiac dysfunction showed controversial results from different animals or experimental models, such as obese rats or rabbits induced by special dietary, genetic obese rats etc. ${ }^{[43-51]}$. There are complex alterations in cardiac structure and function which occur in obesity and obesity-related diseases, including the reduced myofilament calcium sensitivity, changed calcium handling proteins and calcium transients, 
and changed $\beta$-adrenergic pathway ${ }^{[50,52-54]}$. But the mechanism responsible for those changes has not been well understood. Several methods with different species have been used to explore the influences of diabetes on the cardiac function, such as echocardiography, isolated heart perfusion, in situ LV catheterization, LV papillary muscles and magnetic resonance imaging (MRI) ${ }^{[16,55-60]}$. And many mechanisms were also studied to explain the cardiac dysfunction in diabetes mellitus, including the metabolic disorders and structural remodeling caused by hypertrophy, apoptosis, necrosis and fibrosis, changes in the cardiac autonomic neuropathy and calcium handling ${ }^{[57,61-66]}$. Nevertheless, from those evidences, the responsible factors for the myocardial dysfunction in diabetes are still incompletely established.

Although obesity is associated with lower perioperative mortality (mentioned as "obesity paradox"), patients with the metabolic syndrome are exposed to a higher mortality risk during the perioperative period ${ }^{[67]}$. Such obesity paradox has also been reported in critically ill patients ${ }^{[68]}$, including those with severe sepsis ${ }^{[69]}$. However, several large clinical studies challenge the validity of the obesity paradox ${ }^{[70]}$ and obese trauma patients are at risk of higher mortality from persistent hemorrhage ${ }^{[71]}$. In diabetic patients, diastolic dysfunction and a reduced response to $\beta$-adrenoceptor stimulation are observed which may contribute to hemodynamic instability during the perioperative period. Although metabolic syndrome is associated with increasing catecholamine levels and sympathetic activity that chronically stimulates $\beta$-adrenoceptors ${ }^{[72]}$, the $\beta$-adrenoceptor signaling pathway has been inadequately studied in this situation.

Alterations in the $\beta$-adrenoceptor signaling pathway have been observed in senescent ${ }^{[73]}$ and type 1 diabetic rats ${ }^{[55]}$. While an increase in sympathetic nervous system activation is an important mechanism for maintaining cardiac output, the positive inotropic response to $\beta$ adrenoceptor stimulation is markedly altered in type 1 diabetic rats, in part, owing to the down-regulation of $\beta_{1}$-adrenoceptor and the up-regulation of $\beta_{3}$-adrenoceptor, source of a negative inotropic effect. Active efflux transporters, namely the multidrug resistanceassociated protein 4 (MRP4), acts also as an independent endogenous regulator of intracellular cyclic nucleotide levels (3'-5'-cyclic adenosine monophosphate, cAMP), ${ }^{[74]}$ and has been recently shown to be involved in $\beta$-adrenoceptor dysfunction during aging ${ }^{[75]}$.

Zucker obese rat $(\mathrm{fa} / \mathrm{fa})$ is considered as a reliable model of metabolic syndrome. ${ }^{[76]}$ Moreover, this model enables us to study separately obesity and obesity associated with type 2 diabetic status ${ }^{[77]}$. Zucker obese rats develop metabolic syndrome characterized by obesity because of the hyperphagia due to a mutation in the leptin receptor ${ }^{[76,78,79]}$, insulin resistance, hyperinsulinemia, hypertriglycemia and hypercholesterolemia ${ }^{[80]}$. Zucker obese diabetic 
(Zucker diabetic fatty, fa/fa) rats are originated from the selective breeding of Zucker rats with high glucose, which develop hyperphagia due to the nonfunctional leptin receptor, leading obesity and prediabetic state similar to humans ${ }^{[81]}$.

The aim of this study was to compare the $\beta$-adrenoceptor signaling pathway in Zucker lean, Zucker obese, and Zucker obese diabetic rats. This pathway was assessed both in vivo using echocardiography and in vitro using isolated LV papillary muscle. Our hypothesis was that metabolic syndrome is associated with $\beta$-adrenergic dysfunction to some degree, which is aggravated when associated with diabetes. We precisely assessed the effects of $\beta$-adrenergic stimulation on both contraction and relaxation, and particularly focussed on the following possible mechanisms involved, i.e. down-regulation of $\beta_{1}$-adrenoceptors, up- regulation of $\beta_{3^{-}}$ adrenoceptors, and up-regulation of MRP4 $4^{[55,73-75]}$. 


\section{Chapter Two}

\section{Background}

Heart works on the basis of the normal fundamental properties of the heart muscles and numerous modifying, protecting and controlling effects under the nerves, chemicals and mechanical mechanisms on them ${ }^{[82]}$. Control mechanisms are very important to achieve and regulate the physiology of heart. These control mechanisms can be exerted at the central nervous system levels or at the periphery levels, or through the integrative signals transmitted by the local metabolites. Two divisions of the autonomic nervous system, along with their primary messengers / neurotransmitters and two major types of specific receptors, conduct different functions in the cardiovascular reactions ${ }^{[83]}$. The sympathetic nervous system / adrenergic nervous system acts through the adrenergic receptors, while the parasympathetic system acts through the cholinergic receptors. The adrenergic receptors / adrenoceptors are associated with the strengthened contractility and heart rate ( $\beta$-adrenoceptor) or the enhancement of the arteriole tone ( $\alpha$-adrenoceptor). The cholinergic receptors react to their primary messengers, acetylcholine, and exert the opposite effects of adrenergic stimulations. As Rockman, et al. ${ }^{\left[{ }^{84]}\right.}$ concluded that "Adrenergic receptors do not simply genera te second messengers but rather activate a host of signaling proteins and pathways that control cardiac function, myocyte growth and cell death".

There are two main types of adrenergic receptors, $\alpha$-adrenoceptors and $\beta$-adrenoceptors. The density of $\alpha_{1}$-adrenoceptor in human heart is only $10 \%-15 \%$ of the density of $\beta$ adrenoceptor. The maximal positive inotropic effect by the stimulation of $\alpha_{1}$-adrenoceptor is far less than the effects under the stimulation to $\beta$-adrenoceptor ${ }^{[85]}$. $\alpha_{2}$-adrenoceptor in human heart is still not fully understood. However, it has been observed that stimulation to $\alpha_{2}$ adrenoceptor could inhibit the norepinephrine release in the presynaptic component of sympathetic nerve endings in human heart ${ }^{[85,86]}$, thus affecting the plasma norepinephrine levels, especially in patients with heart failure. $\beta$-adrenergic receptors are class of $\mathrm{G}$ proteincoupled receptors, with three subtypes $\beta_{1}$-adrenoceptor, $\beta_{2}$-adrenoceptor and $\beta_{3}$-adrenoceptor. $\beta_{1}$-adrenoceptor subtype is the main cardiac $\beta$-adrenergic receptor, while most $\beta_{2^{-}}$ adrenoceptors are noncardiac receptor ${ }^{[87]}$. Although they have different distributions within different tissues, organs and species, they can co-exist in the same ventricular cell and exert the positive inotropic responses ${ }^{[88]}$. In the human heart, $\beta$-adrenoceptors, such as $\beta_{1}$ - 
adrenoceptor and $\beta_{2}$-adrenoceptor, play important roles not only in the physiological status, but also in diseased conditions. Different cardiac receptors related pathways participate in the regulation of cardiac performances: some receptors work via the Gs protein - adenylyl cyclase pathway, such as $\beta$-adrenoceptors and histamine receptors; some work via the Gi protein adenylyl cyclase pathway, such as muscarinic receptors and adenosine receptors; and some others work via $\mathrm{G}_{\mathrm{q} / 11}$ protein - phospholipase $\mathrm{C}$ - protein kinase $\mathrm{C}$ pathway, including $\alpha_{1^{-}}$ adrenoceptor and angiotension II receptors ${ }^{[85]}$.

\section{Physiological and pathophysiological $\beta$-adrenergic receptor signaling pathway in the heart}

1.1 The physiological characteristics of $\beta$-adrenergic receptors signaling pathway in the heart of animals and human

In human heart, $\beta_{1}$-adrenoceptor and $\beta_{2}$-adrenoceptor co-exist, $\beta_{1}$-adrenoceptor being predominant ${ }^{[89]}$. Both $\beta$-adrenoceptor subtypes couple to Gs protein, increase the cAMP levels and induce the positive inotropic and chronotropic effects in vivo, and in vitro. In atria, stimulation to $\beta_{1}$-adrenoceptor and $\beta_{2}$-adrenoceptor can cause maximal physiological effects. But in ventricles, only stimulation to $\beta_{1}$-adrenoceptor can evoke maximal effects, and stimulation to $\beta_{2}$-adrenoceptor causes submaximal results ${ }^{[89]}$. In rats and murines hearts, stimulation to $\beta_{1}$-adrenoceptor can induce the positive inotropic and chronotropic effects, but facilitate the cardiomyocytes apoptosis as well ${ }^{[90-94]}$.

The post-receptor signaling pathway of $\beta_{1}$-adrenoceptor is widely clarified to conduct the positive inotropic effects, the lusitropic / relaxant effects, the chronotropic effects and the dromotropic effects under the $\beta$-adrenergic stimulation ${ }^{[82]}$. The cardiac inotropic effect is regulated mostly by the amount of calcium ions entry into cytosol during the process of activation. $\beta$-adrenergic stimulation can enhance the force of contraction (positive inotropic effect) and the rate of relaxation (lusitropic effect), then change the pattern of contraction and relaxation (Fig. 2-1) ${ }^{[82]}$. 


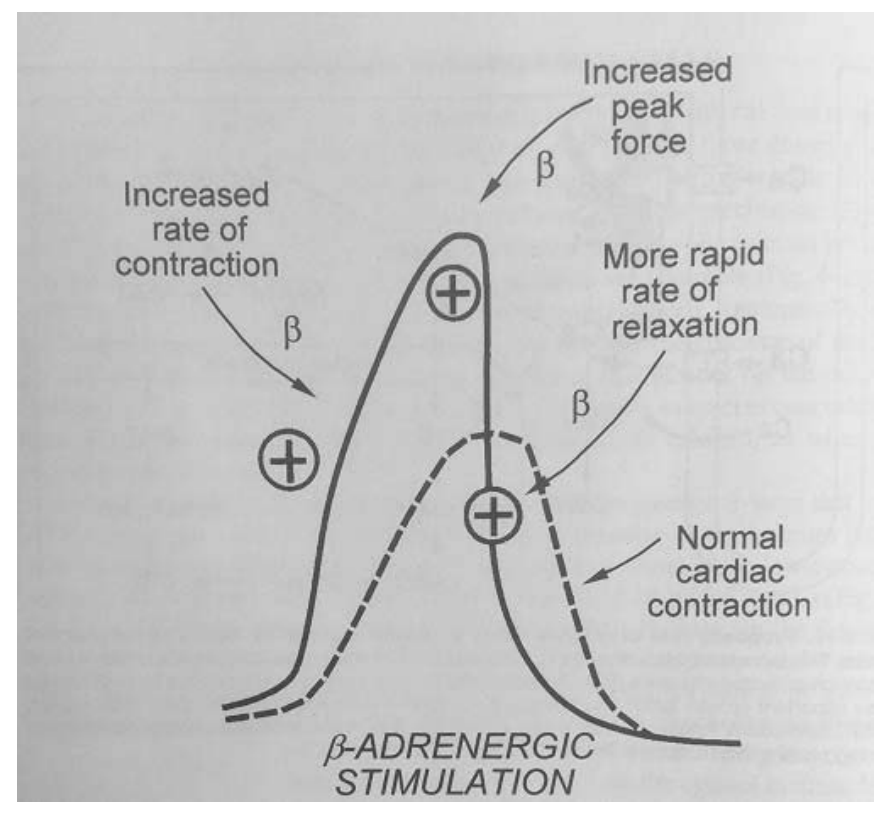

Fig. 2-1 The effects of $\beta$-adrenergic stimulation on the pattern of cardiac contraction. Reproduced from Opie ${ }^{[82]}$

$\beta$-adrenergic stimulation to $\beta$-adrenoceptor induces molecular changes, then the binding of Gs protein to guanosine $5^{\prime}$ - triphosphate (GTP) catalyses subunit of adenylyl cyclase to produce cAMP from adenosine 5' - triphosphate (ATP). Positive inotropic effect in response to $\beta$-adrenergic stimulation involves some pathways: 1) $\beta$-adrenergic stimulation increases cAMP-mediated activation of protein kinase A (PKA) that subsequently increases the phosphorylation of sarcolemmal protein of the calcium channels by PKA; thereby increases the inward calcium current $\left(\mathrm{I}_{\mathrm{ca}}\right)$, causing a great rate of calcium-induced calcium ions release through the ryanodine receptor (RyR). 2) $\beta$-adrenoceptor stimulation increases cAMPmediated activation of PKA that subsequently increases the phosphorylation of phospholamban (PLB), enhancing re-uptake of calcium into the sarcoplasmic reticulum (SR); then, preloading the SR with more calcium ions will increase the amount of calcium ions released in response to any amount of trigger calcium; thus, the contractile response will be further stimulated ${ }^{[95]} .3$ ) increase of intracellular free calcium ions enhances de - inhibition of actin and myosin by interaction of calcium with troponin $\mathrm{C}$, and promotes actin-myosin interaction; 4) increase in intracellular free calcium ions accelerates splitting of ATP by myosin ATPase to increase the rate of development of contractile forces; thus, $\beta$-adrenoceptor stimulation increases the crossbridge cycling ${ }^{[22,96]}$.

$\beta$-adrenergic stimulation can also enhance the rate of relaxation (lusitropic effect), then change the pattern of contraction and relaxation ${ }^{[82]}$. Lusitropic effect is mostly induced at a 
subcellular level by the increased activity of calcium pump in the SR in response to the phosphorylation of phospholamban by cAMP and PKA. Sarco(endo)plasmic reticulum $\mathrm{Ca}^{2+}-$ ATPase (SERCA) is a calcium uptake pump of sarcoplasmic reticulum, constituting about $40 \%$ of the protein component of SR with the major mechanism of reducing the cytosolic calcium ion level and initiating diastole ${ }^{[82]}$. In the rabbit myocardium, nearly $75 \%$ of the activator calcium is removed by calcium uptake pump of SR, and sodium / calcium exchanger (NCX) removes nearly $25 \%$, and only about $1 \%$ is removed by the calcium pump of sarcolemma or transported into mitochondria ${ }^{[97]}$. Thus, the most majority of calcium released from SR return to its origin site by the activity of SERCA. There are three different genes encoding for SERCA, among which the SERCA2a is predominately expressed in cardiac tissues (cardiac isoform, SERCA2a) ${ }^{[98]}$. The activity of SERCA2a depends on the amount of SERCA2a proteins, and is normally inhibited by phospholamban ${ }^{[99]} \cdot \beta$-adrenergic stimulation can relieve this inhibition via cAMP and PKA phosphorylating phospholamban, therefore the calcium uptake is stimulated. Lusitropic effects of $\beta$-adrenergic stimulation involve several pathways: 1) $\beta$-adrenergic stimulation, via cAMP and PKA, phosphorylates phospholamban, increases the turnover and sensitivity of SERCA2a, and further increases the removal of calcium out of cytosol; 2) phosphorylation of plasma member sodium pump could increase the calcium efflux via sodium/calcium exchanger; 3) phosphorylation of troponin I could reduce the calcium binding through decreasing the calcium sensitivity to the troponin I complex, and further increase the rate of crossbridge detachment. 4) increased intracellular calcium level, via calmodulin and calmodulin-dependent kinase (CaMK), enhances the phosphorylation of phospholamban directly ${ }^{[82,96]}$. Thus, the contraction and relaxation rates are both enhanced, which are the positive inotropic effects and the positive lusitropic effects. $\beta_{1}$-adrenoceptor / Gs protein coupling pathway can also evoke PKA-independent, CaMK II mediated apoptotic procedure. Figure 2-2 demonstrates the signaling pathway of $\beta_{1}$ adrenoceptor and $\beta_{2}$-adrenoceptor in cardiomyocytes ${ }^{[100]}$. 


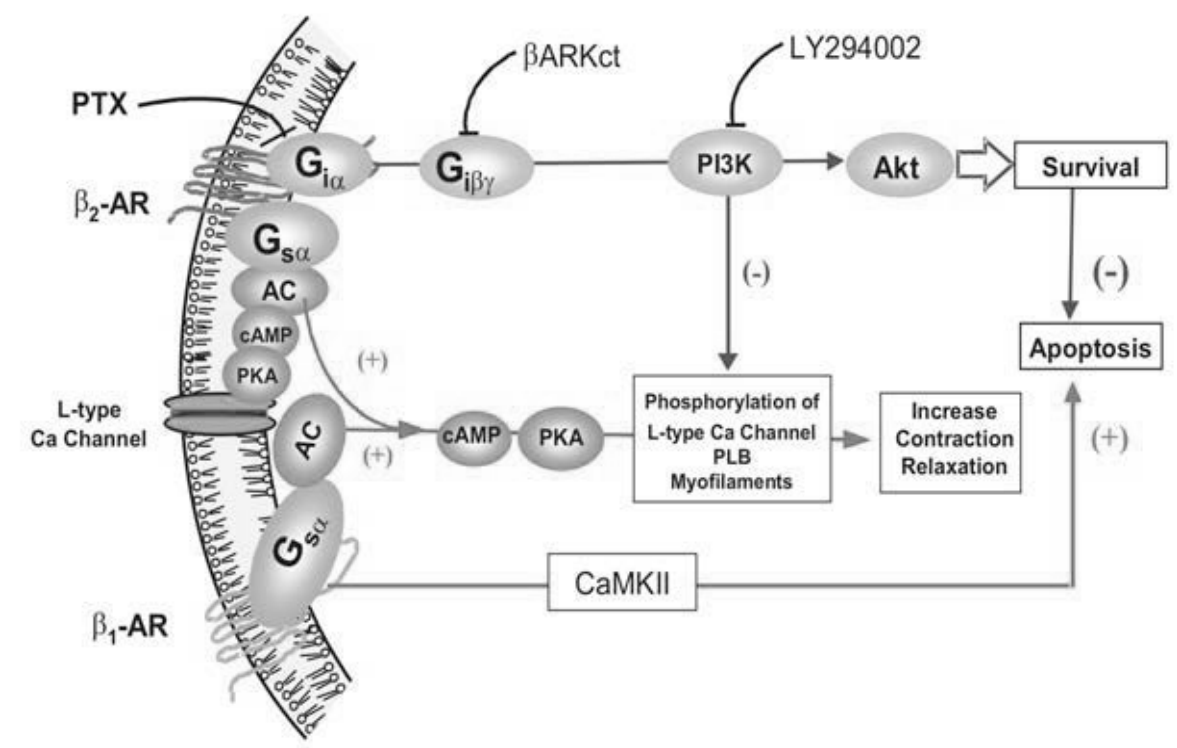

Fig. 2-2 Signaling pathway of $\beta_{1}$-adrenoceptor and $\beta_{2}$-adrenoceptor in cardiomyocytes. $\beta A R K-c t$, a peptide inhibitor of Gi $\beta \gamma$ signaling; LY294002, a PI3K inhibitor. PTX: pertussis toxin; $\beta$ ARK: $\beta$-adrenergic receptor kinase; PI3K: phosphatidylinositol 3-kinase; Akt: protein kinase B; AC: adenylyl cyclase; cAMP: 3',5'-cyclic adenosine monophosphate; PKA: protein kinase A; PLB: phospholamban; CaMK II : calmodulin-dependent kinase II . Reproduced from Zheng et al. ${ }^{[100]}$

The role of cardiac $\beta_{2}$-adrenoceptor has still not been fully understood. One early research, using transgenic mice of over - expressing human $\beta_{2}$-adrenoceptor (TG4 mice), first confirmed the biochemical evidences that $\beta_{2}$-adrenoceptor was coupled to both Gs protein and Gi protein ${ }^{[101]}$. The ventricular myocytes of wild - type (WT) and TG4 mice did not develop the increased contractile responses to the stimulation of $\beta_{2}$-adrenoceptor agonists, unless the Gi protein activity was inhibited by pertussis toxin (PTX). That was to say that the activation of $\beta_{2}$-adrenoceptor - Gi protein system would preclude the positive inotropic responses under the $\beta_{2}$-adrenoceptor stimulation. In WT mice pretreated with $\beta_{1}$-adrenoceptor blockade or in $\beta_{1}$-adrenoceptor knock-out mice, stimulation of isoproterenol (a mixed agonist) could not induce positive inotropic responses ${ }^{[102,103]}$. These results indicate that the positive inotropic responses under isoproterenol were mostly attributed to $\beta_{1}$-adrenoceptor stimulations in WT mice cardiac myocytes or in $v i v{ }^{[102,104,105]}$, whereas $\beta_{2}$-adrenoceptor - Gi protein coupling induced a negative feedback to $\beta$-adrenoceptor stimulations. Because of the concurrent coupling to Gi protein, $\beta_{2}$-adrenoceptor seems dormant and shows no significant function in the regulation of contractions in cardiac myocytes or myocardiums of mice and other 
mammalian species ${ }^{[88,101,103,106]}$. Nevertheless, $\beta_{2}$-adrenergic stimulation can still function via the Gs protein - adenylyl cyclase - cAMP - PKA pathway, and exert the positive inotropic and lusitropic effects similar to the $\beta_{1}$-adrenoceptor stimulations. In all, $\beta_{2}$-adrenoceptor is coupled to Gs protein and Gi protein in rats and murines, and can cause anti - apoptotic effects $^{[90-94,107-109]}$. In isolated human atrium and ventricular myocardium, activation of $\beta_{2^{-}}$ adrenoceptor can induce similar effects as the stimulation to $\beta_{1}$-adrenoceptor, including increased contractile force, and enhanced relaxation through cAMP dependent phosphorylation of phospholamban and troponin $\mathrm{I}^{[110-113]}$. These evidences show that $\beta_{1}$ adrenoceptor and $\beta_{2}$-adrenoceptor in human heart are both coupled to Gs protein. However, whether $\beta_{2}$-adrenoceptor is coupled to Gi protein as in rats and murines hearts, it is still in debate.

Researchers found that different $\beta_{2}$-adrenoceptor agonists may activate different fashion of pathway, either $\beta_{2}$-adrenoceptor - Gs protein pathway or $\beta_{2}$-adrenoceptor - Gs protein and $\beta_{2}$ adrenoceptor - Gi protein pathways ${ }^{[114-116]}$. An early study found that nebulised $\beta_{2^{-}}$ adrenoceptor agonist could cause different cardiovascular effects in healthy human heart, which showed that fenoterol induced significantly greater chronotropic electrocardiographic and inotropic effects than terbutaline did ${ }^{[117]}$. In animal experiment, terbutaline was observed to couple to Gs protein and Gi protein, and fenoterol only coupled to Gs protein ${ }^{[16]}$. Another research found that a $\beta_{2}$-adrenoceptor antagonist (ICI118,551) worked as the agonist to Gi protein in ventricular cardiomyocytes of human failing heart (increased Gi protein activity in failing heart), which was the direct negative inotropic effects, whereas this did not occur in non-failing human heart (normal Gi protein activity in healthy heart) ${ }^{[118]}$. Besides, $\beta_{2^{-}}$ adrenoceptor coupled to the inhibitory G protein Gi can activate the phospholipase A2 / arachidonic acid pathway ${ }^{[119,120]}$. The coupling of $\beta_{2}$-adrenoceptor to the inhibitory protein Gi also activates the Gi protein - PI3K - Akt pathway, which can compartmentalize and counteract the Gs protein - adenylyl cyclase - cAMP - PKA signaling pathway ${ }^{[100]}$. Thus cardiac $\beta_{2}$-adrenoceptor - Gi protein pathway might be more important in rodents than in human. In the failing heart, the percentage of $\beta_{2}$-adrenoceptor may double as that in the normal ventricle, while the $\beta_{1}$-adrenoceptor down-regulates. It has been suggested that the Gi pathway is associated with an anti - apoptotic mechanism which benefits in the failing heart ${ }^{[121,122]}$. Therefore, $\beta_{2}$-adrenoceptor is regarded to work via both the stimulatory G protein Gs and the inhibitory $\mathrm{G}$ protein $\mathrm{Gi}$, with the former dominating physiologically and the latter dominating pathologically (Fig. 2-2 $)^{[100,108]}$. 
$\beta_{3}$-adrenoceptor mainly functions in the adipose tissues, and also acts in the heart. Unlike $\beta_{1}$-adrenoceptor and $\beta_{2}$-adrenoceptor, the cardiac $\beta_{3}$-adrenoceptor is lack of the PKA phosphorylation site and has fewer serine / threonine residues in the $\mathrm{C}$ - terminus tail ${ }^{[123]}$. So, under the sustained adrenergic stimulations or high catecholamine levels, the $\beta_{3}$-adrenoceptor may preserve the responses, whereas $\beta_{1}$-adrenergic and $\beta_{2}$-adrenergic responses may diminish them $^{[119,124]}$. In human, the cardiac $\beta_{3}$-adrenergic stimulations were found to reduce the cardiac contractility and induce a negative inotropic response, but a positive chronotropic effect $^{[119,125,126]}$. However, this positive chronotropic effects in vivo by $\beta_{3}$-adrenergic stimulation could be inhibited by $\beta_{1}$-adrenoceptor and $\beta_{2}$-adrenoceptor blockades, implying that these effects might be attributed to the baroreflex activation resulting from the vasodilation induced by $\beta_{3}$-adrenergic stimulation ${ }^{[127]}$. The concentration - dependent negative inotropic effects were observed under different $\beta_{3}$-adrenergic stimulations in rabbit ventricular cardiomyocytes and rat Langendorff - perfused heart ${ }^{[128-130]}$. Meanwhile, the $\beta_{3^{-}}$ adrenergic stimulation was found to induce a negative lusitropic effect, and counteract the positive lusitropic effect by isoproterenol in rats ${ }^{[130]}$. These suggest that the lusitropic effects by $\beta_{3}$-adrenergic stimulation would counteract the excessive responses by $\beta_{1}$-adrenergic and $\beta_{2}$-adrenergic stimulation to keep a normal cardiac function. The cardiac effects of different $\beta_{3}$-adrenergic stimulations are also variable among different tissues and species. The responses of contractility by $\beta_{3}$-adrenergic stimulations are significantly different between artria and ventricles in rats, but not in human ${ }^{[119,124]}$. Absence or very low effects of $\beta_{3}$ adrenergic stimulations were observed in rats atria ${ }^{[119]}$. In the neonatal rats cardiomyocytes, there are also lack of responses to $\beta_{3}$-adrenergic stimulation ${ }^{[131]}$. Almost no $\beta_{3}$-adrenoceptor expression in heart was observed in rat myocardium ${ }^{[132]}$. The attenuation of cardiac responses to the $\beta_{3}$-adrenergic stimulations is suggested to involve the alterations in the excitation contraction coupling and transmembrane ions channels activities ${ }^{[19]}$. The post - receptor signaling pathway of $\beta_{3}$-adrenoceptor in heart is not coupled to Gs protein, but the Gi protein (Fig. 2-3). In rodent adipocytes, $\beta_{3}$-adrenoceptor is coupled to both Gs and Gi proteins ${ }^{[133,134]}$. In human ventricular myocardium, the activation of $\mathrm{Gi}$ protein causes the activation of the nitric oxide (NO) pathway rather than the inhibition of adenylyl cyclase, suggesting that the $\beta_{3}$-adrenoceptor - NO - guanylyl cyclase - cyclic guanosine monophosphate (cGMP) pathway may function as a negative feedback to the positive inotropic effects under the $\beta$-adrenergic stimulations in heart ${ }^{[126,135]}$. And NO could also mediate the cardiac responses in a cGMP independent way through the covalent modifications of the key proteins ${ }^{[119]}$. Nevertheless, the 
negative inotropy induced by the cardiac $\beta_{3}$-adrenoceptor still remains subtle in healthy tissues $^{[136]}$.

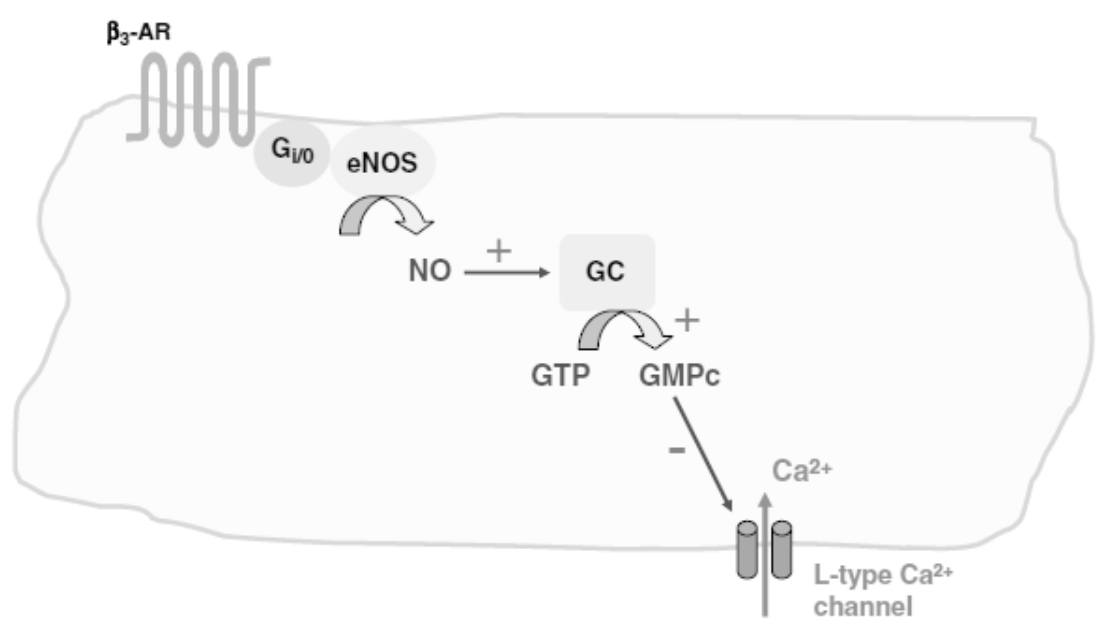

Fig. 2-3 Signaling pathway of $\beta_{3}$-adrenoceptor in cardiomyocytes. NOS: nitric oxide synthase; NO: nitric oxide; GC: guanylate cyclase; cGMP: 3',5'-cyclic guanosine phosphate. Reproduced from Rozec et al. ${ }^{[119]}$

Additionally, a "putative $\beta_{4}$-adrenoceptor", identified as a low - affinity state of $\beta_{1}$ adrenoceptor ( $\beta_{1 \mathrm{~L}}$-adrenoceptor), was found to exert positive inotropic effects and hastening relaxation through Gs protein - cAMP - PKA pathway under the stimulation of non conventional partial agonist ${ }^{[137-139]}$. These agonists, originally as $\beta_{1}$-adrenoceptor and $\beta_{2^{-}}$ adrenoceptor antagonists, induced the stimulant effects at a higher concentration than the blockade concentration to $\beta_{1}$-adrenoceptor and $\beta_{2}$-adrenoceptor ${ }^{[137]}$.

$\beta$-adrenoceptor genetic polymorphisms play important roles in cardiac function, with two functional important single nucleotide polymorphisms (SNPs) in $\beta_{1}$-adrenoceptor gene: Ser49Gly and Arg389Gly; and three important SNPs in $\beta_{2}$-adrenoceptor gene: Arg16Gly, Gln27Glu and Thr164Ile ${ }^{[85]}$. Although still conflicting, it seems that they act as risk factors rather than disease causing genes in cardiovascular diseases, which could exert influences on the development and progress of disease.

1.2 The pathophysiological changes of $\beta$-adrenergic receptor signaling pathways in the heart of two commn diseases

The state of aging or chronic heart failure (CHF) both facilitate sympathetic activity, which shows an increase of plasma norepinephrine / epinephrine levels but a diminished functional responses to $\beta$-adrenergic stimulations. The employment of $\beta$-blockers, including carvedilol, 
metoprolol, bisopolol and nebivolol, has shown beneficial effects on the survival and left ventricular remodeling in the chronic heart failure ${ }^{[140,141]}$. It further confirms the state of enhanced adrenergic drive in the development of cardiac dysfunction. Although the exact mechanisms still remain unclear, functions of $\beta$-adrenoceptor signaling pathway might change as following under both diseased conditions: 1) deficiency of the excitation - contraction coupling mechanism; 2) down - regulation of $\beta_{1}$-adrenoceptor, including reduced $\beta_{1^{-}}$ adrenoceptor density; 3) changes in the $\beta$-adrenoceptor - $G$ protein pathway, including uncoupling or reduced reaction to the Gs protein - adenylyl cyclase - cAMP pathway, and enhanced Gi protein; 4) an up - regulation of G protein-coupled receptor kinase (GRK) and $\beta$ adrenoceptor desensitization; 5) a decreased re-uptake of neuronal uptake transporter ${ }^{[55,142]}$.

As mentioned about Gi protein, at least in rats, stimulations to cardiac $\beta$-adrenoceptor can exert proapoptotic effects via $\beta_{1}$-adrenoceptor - Gs protein pathway, and anti - apoptotic effects via $\beta_{2}$-adrenoceptor - Gi protein pathway ${ }^{[142,143]}$. Meanwhile, cardiac Gi protein might protect patients with chronic heart failure from catecholamine-related arrhythmias ${ }^{[144,145]}$. However, the influences of Gi protein in patients with chronic heart failure are still under explorations.

In aging heart, the reduced responsiveness to $\beta$-adrenoceptor stimulation is associated with the deficiency in the $\beta$-adrenoceptor signaling pathways of both $\beta_{1}$-adrenoceptor and $\beta_{2}$ adrenoceptor, while the primary characteristics of excitation - contraction coupling is unchanged $^{[142]}$. Although conflicting results were observed in the selective reduction of myocardial $\beta_{1}$-adrenoceptors and $\beta_{2}$-adrenoceptor in aging heart, some research still confirmed non - selective reduced $\beta$-adrenoceptor density in aged rats ventricular myocytes and myocardium ${ }^{[142,146]}$. In senescent rats, the down - regulation of $\beta_{1}$-adrenoceptor and $\beta_{2}$ adrenoceptor, and the up - regulation of $\beta_{3}$-adrenoceptor have also been confirmed ${ }^{[73,142]}$. This change is different from the condition in the failing heart, predominately down - regulation in $\beta_{1}$-adrenoceptor but almost no change in $\beta_{2}$-adrenoceptor density ${ }^{[147-149]}$. Meanwhile, the age related reduction in the cardiac adenylyl cyclase activity might also contribute to the decreased responses to agonists of both $\beta$-adrenoceptors. The sarcolemmal L - type calcium channel was same between ventricular myocytes of young and aged rats ${ }^{[150]}$. Similar to the hypertrophic or failing heart, the reduced contractile responses to $\beta_{2}$-adrenoceptor stimulations was observed due to the reduced amplification function of SR in the aged rats. Considering the changes of Gi protein, there were also different findings in rats, but some researchers still confirmed that age - related changes of $\beta$-adrenoceptor signaling system were not associated with the biochemical or functional alterations of pertussis toxin (PTX) - 
sensitive $G$ protein (Gi protein) ${ }^{[142,151-153]}$. This is also different from the changes in the failing heart, which demonstrated the elevated abundance and functional activity of Gi protein $[154,155]$

Additionally, $\beta$-adrenergic receptor kinase (a GRK), is up - regulated and can promote the binding of $\beta$-arrestins to the phosphorylated receptor in the failing hearts and hypertrophic hearts ${ }^{[156-158]}$. This causes the uncoupling of $\beta$-adrenoceptor to Gs protein pathway and consequently the decreased responsiveness of $\beta$-adrenoceptor to the agonist stimulation ${ }^{[85]}$. Actually, for most of the $\mathrm{G}$ protein coupled receptors, increased exposure to the stimulations by agonists would lead to a reduction in responsiveness of receptors, which is called desensitization. This desensitization might be initiated by the phosphorylation of GRK family to the activated receptors ${ }^{[142]}$. Among which, $\mathrm{GRK}_{2}$ is the most common isoform and crucial to myocardial function. However, the role of the GRKs is still not totally clarified in human heart. The major stimuli to activate $\mathrm{GRK}_{2}$ are $\beta$-adrenoceptor stimulations. In fact, some animal and human experiments observed the decreases of $\mathrm{GRK}_{2}$ activation under $\beta$ adrenoceptor blockades in failing heart ${ }^{[89,159]}$. In animal models of heart failure, a $\mathrm{C}$ - terminal domain of $\mathrm{GRK}_{2}$ ( $\beta \mathrm{ARKct}$ ) was found to inhibit $\mathrm{GRK}_{2}$ activity, reduce cardiac hypertrophy and reduce the progressive worsening of myocardial function ${ }^{[160,161]}$. However, in aging heart, no changes of $\mathrm{GRK}_{2}$ and its activity were found ${ }^{[142,162]}$.

\section{Obesity and diabetes mellitus related cardiomyopathy and the alterations in the $\beta$-adrenergic receptor signaling pathway}

2.1 Obesity related cardiac dysfunctions and alterations in the $\beta$-adrenergic receptor signaling pathway

Obesity has been paid ever-increasing attentions not only for the obesity influences per se, but also for its prevalent co - morbidities, including diabetes mellitus and cardiovascular disease $^{[124,163]}$. In the obese human, there are cardiac hypertrophy, hyperkinetic circulation and cardiac dysfunction ${ }^{[164-166]}$. Cardiovascular dysfunction in the clinically severe obesity was first reported in the obese volunteers through cardiac catheterization, who showed decreased left ventricular compliance and stroke work index, and increased LV end-diastolic pressure $^{[167]}$.These abnormalities are all correlated to the severity of obesity ${ }^{[167]}$. And the correlations between obesity and left ventricular mass, as well as the correlations between body weight and diastolic dysfunction, have all been confirmed ${ }^{[168,169]}$. In the young healthy 
obese women, the systolic function, diastolic function, and cardiac efficiency are all decreased $^{[170,171]}$.

As in obese human, Zucker obese rats showed declined left ventricular functions ${ }^{[172]}$. The contractile dysfunction has been observed in several studies in Zucker obese rats or obese rabbits $^{[43,54,173,174]}$. Zucker obese rats were shown to develop diastolic dysfunction with preserved left ventricular ejection fraction since 9 weeks of age ${ }^{[175,176]}$. This is in accordance with the finding in isolated hearts of obese rabbits shown since a decrease in maximal rate of pressure rise $(+d p / d t)$ and the maximal rate of pressure decline $(-d p / d t)$ in response to the stimulation of forskolin has been observed ${ }^{[96]}$. In young Zucker obese rats (12 - 15 weeks of age), an increased LV end - systolic pressure ${ }^{[81]}$, or higher LV developed pressure has been observed $^{[177,178]}$. Some researchers observed unchanged LV fractional shortening, cardiac output, LV end - systolic pressure and LV end - systolic pressure - volume relation, along with increases in the LV end - diastolic pressure, LV relaxation constant Tau and LV end diastolic pressure - volume relation in the Zucker obese rats at 24 weeks of age ${ }^{[172]}$. These results demonstrate that the LV diastolic function is impaired but with relatively preserved systolic function during obesity. A recent study used hypercaloric - diet Wistar rats for 30 weeks which exhibit obesity, slight hyperglycemia, hyperinsulinemia and hyperleptinemia, but no differences in protein and lipid levels ${ }^{[7]}$. Meanwhile, these obese Wistar rats showed no structural remodeling or changes in heart rate, systolic arterial blood pressure, and performances of LV papillary muscles after inotropic maneuvers (myocardial stiffness, postrest contraction, increase in extracellular calcium concentration, and change in heart rate). These results are similar with previous studies, including isolated hearts of obese rabbits with high - fat diet for 12 weeks and isolated cardiac myocytes of obese Sprague - Dauley rats with high - carlorie diet for 14 weeks $^{[47,49]}$, but different with diverse general characteristics and / or reduced myocardial mechanical functions in obese animals of other studies ${ }^{[10,48,179-182]}$. Meanwhile, in the research of LV papillary muscles in vitro, there were also functional impairments in response to the progressively higher extracellular concentration of calcium and post-rest contraction stimulus, shown by the lower responses of peak developed tension and the lower maximum speed of the negative variation in developed tension $(-\mathrm{dT} / \mathrm{dt})$ in obese Wistar rats ${ }^{[44]}$. In the obese hypertensive rabbits experiment, responses to $\beta$-adrenoceptor stimulation were decreased both in isolated heart and isolated papillary muscles ${ }^{[43]}$.

Cardiac remodeling has been proposed as an adaptive trait of obesity ${ }^{[183]}$. It has been observed in many researches about obesity, especially in long - term obesity ${ }^{[50,179,184,185]}$. It could be elicited directly by the increases in cardiac preload and afterload, and indirectly 
through the cardio - metabolic changes related to obesity, including dyslipidemia, insulin resistance and diabetes ${ }^{[186,187]}$. As a core abnormality of obesity, insulin resistance, along with hyperinsulinemia, could facilitate cardiac remodeling through the growth - promoting property of insulin, or through reduced anti - apoptotic signaling pathway (phosphatidylinositol 3 - kinase (PI3-K) / Akt pathway) evoked by the activation of insulin receptor ${ }^{[188]}$. The cardiac remodeling in obesity may significantly influence the cardiovascular functions. Some animal experiments have shown a decreased or unaltered systolic function through echocardiography ${ }^{[45,189,190]}$. One observation, in the long - term high - fat diet induced obese Wistar rats, showed elevated systolic function, including the increased endocardial and midwall fractional shortenings and posterior wall shortening velocity (PWSV) ${ }^{[179]}$. One explanation of these findings was the decreased afterload and left ventricular contractility improvement. The diastolic dysfunction in obesity could be found as a higher A wave and a lower E/A ratio using echocardiography, and the prolongation of isovolumic relaxation time (IVRT), which directly shows an impairment of cardiac filling ${ }^{[191-196]}$. Diastolic filling includes the active relaxation associated with calcium handling, and the passive properties of myocardium related to the viscoelastic characteristics ${ }^{[97,197-200]}$. The diastolic dysfunction might be attributed to the metabolic abnormalities (insulin resistance, hyperinsulinemia and hyperglycemia), hemodynamic changes, and / or calcium handling homeostasis in obesity $^{[201,202]}$. However, a diet with moderate fat in the obesity - prone Sprague - Dawley rats was only found to induce higher body weight, body fat gain, serum leptin and cholesterol ${ }^{[45]}$. In these obesity - prone rats, no differences were observed in the arterial blood pressure, left ventricular systolic or diastolic function.

The basic mechanisms resulting in the cardiac dysfunction in obesity are still under debate, including a changed $\beta$-adrenergic signaling pathway. At first, researchers found that reduced $\beta$-adrenoceptor density was associated with the impaired contractile responses ${ }^{[54]}$. This early experiment in Zucker obese rats aged 22 weeks showed that the impairment in response to $\beta$ adrenoceptor stimulation was associated with both reduction in the number of $\beta$ adrenoceptors and changed coupling between $\beta$-adrenoceptor and Gs protein in heart tissue membranes. Later, conflicting results were published, suggesting that the impaired inotropic responses in obesity occurred with unchanged $\beta$-adrenoceptor expression ${ }^{[203,204]}$. Experiments in obese rabbits did not demonstrate differences in $\beta$-adrenoceptor density or affinity ${ }^{[204]}$. In the cardiomyocytes of $o b / o b$ mice, the abundance of $\beta_{1}$-adrenoceptor and $\beta_{2}$-adrenoceptor was not altered ${ }^{[203]}$. In obese hypertensive rats experiments, a decreased response to $\beta$ adrenoceptor stimulation was observed together with a decreases in $\beta$-adrenoceptor number 
and affinity ${ }^{[174]}$. The decrease in $\beta$-adrenoceptor number might also be attributed to slight hypothyroidism in Zucker obese rats, since the thyroid hormone could elevate the $\beta$ adrenoceptor levels ${ }^{[205]}$. Responses to $\beta$-adrenoceptor stimulation was reduced both in isolated heart and LV papillary muscles in the obese hypertensive rabbits ${ }^{[43]}$, and the possible defect site may lie in post- $\beta$-adrenoceptor signaling pathways ${ }^{[96]}$.

The post- $\beta$-adrenoceptor signaling pathways include series of components and cascade reactions. The switching of Gs and Gi proteins mediated the isoproterenol - induced cardiac hypertrophy in neonatal rats ${ }^{[206]}$. In the heart of $o b / o b$ mice, Gs protein and Gi protein levels were both reduced, among which only Gs $\alpha$ protein $(52 \mathrm{kDa})$ could be restored after leptin replenishments. It has been supported that, in the $\mathrm{G}$ proteins stimulating adenylyl cyclase, the long splice Gs $\alpha$ protein - 52kDa showed greater potency and more basic activity than the short splice Gs $\alpha$ protein $-45 \mathrm{kDa}^{[207]}$. The activity of adenylyl cyclase of the cardiomyocytes in obesity shows different findings. The activity of adenylyl cyclase in the heart of diet induced obese rabbit or $o b / o b$ mice was unaltered ${ }^{[203,204]}$. The findings in the $o b / o b$ mice (10 weeks of age) demonstrated significantly impaired cardiac $\beta$-adrenergic inotropic responses as depressed responses to forskolin and dibutryl cAMP, whereas preserved adenylyl cyclase activity and reduced PKA activity were observed ${ }^{[203]}$. Further, experiments in obese rabbits did not demonstrate differences in the adenylyl cyclase activity or coupling of the $\beta$ adrenoceptor to adenylyl cyclase activation ${ }^{[204]}$. At the same time, the decreased response to $\beta$-adrenoceptor stimulation was also observed to be associated with reduction of cAMP production and SR calcium uptake in the obese hypertensive rats ${ }^{[174]}$. The cardiac adenylyl cyclase activity of Zucker obese rats under the stimulation to the $\beta$-adrenoceptors exhibited a marked age - dependent reduction ${ }^{[205]}$. One research ${ }^{[174]}$, using ventricular myocytes of female spontaneous hypertension-heart failure(SHHF)/Mcc- $c p$ and JCR:LA-cp rats, found that obesity per se would not influence the accumulation of calcium in SR, but could reduce the cAMP production. Nevertheless, if obesity is associated to hypertension, SR calcium accumulation and cAMP production would both be depressed. PKA is very important in the regulation to the downstream of cAMP mediated phosphorylations in the $\beta$-adrenergic signaling pathway ${ }^{[82]}$, including the phosphorylation of PLB, which is pivotal for removing the inhibition of PLB on SERCA2a, allowing increases in calcium re-uptake, and consequently increased contractility. The PKA activity in the $o b / o b$ mice was depressed, and the production of phospho - phospholamban (P-PLB) was reduced ${ }^{[203]}$. Meanwhile, in the $o b / o b$ mice, SR calcium stores were depressed even under the seemingly compensatory up regulated SERCA2a expressions ${ }^{[203]}$. 
Obesity related cardiac dysfunction is also related to the alterations in the intracellular calcium handling. Several studies in obese Wistar rats induced by hypercaloric diet for 15 weeks, showed cardiac dysfunction ${ }^{[44,51]}$. This dysfunction suggested that obesity might impair the regulatory calcium channels in the myocardium, especially the sodium / calcium exchanger, L-type calcium channels of the sarcolemma, SR and myofilaments sensitivity to calcium $^{[44,163]}$. Besides, the decreased maximum speed of the positive variation in developed tension $(+\mathrm{dT} / \mathrm{dt})$ under the $\beta$-adrenergic stimulation was observed with a decreased phosphorylation of phospholamban via calcium - CaMK in obese Wistar rats ${ }^{[44]}$. In high calorie diet induced obese Sprague - Dawley rats, there was also a cardiac contractile depression due to the reduced phosphorylation of phospholamban, even though the increases of proteins expression in SERCA2a and phospholamban were found ${ }^{[50]}$. Meanwhile, the unchanged proteins expression of SERCA2a, RyR2 and phospholamban was observed in non - obese rats with high - calorie diet ${ }^{[48]}$, which further confirmed the influences of obesity, not just high fat dietary, on the calcium related proteins expression. In hypercaloric - diet Wistar rats (for 30 weeks), no changes of responses under the inotropic stimulations was noted ${ }^{[7]}$. The possible reasons for non - significant inotropic stimulation effects in those long - term obesity rats might be the unaffected intracellular calcium - cycling related proteins levels (L type calcium channel, SERCA2a, calsequestrin), and further the preserved intracellular calcium entry and resequestration even when the down - regulation of phospholamban phosphorylation at Ser16 was observed ${ }^{[179]}$. The cardiac $\beta$-adrenergic inotropic responses were impaired in the $o b / o b$ mice at 10 weeks of age ${ }^{[203]}$. This isolated cardiac myocytes model showed reduced sarcomere shortening and calcium transient under the stimulation of isoproterenol, and decreased calcium stores in SR in the obese mice. They also observed decreased expressions of Gs $\alpha$ protein, Gi $\alpha$ proteins and P-PLB, and increased expressions of SERCA2a. After replenishment of leptin to the cardiac myocytes, the inotropic responses, PKA activity, levels of P-PLB and SR calcium strores could be restored back to the control level ${ }^{[203]}$. These results suggest that the role of PKA activity on the SR calcium cycling dysfunction, and the leptin - deficiency and resistance might be the cause of cardiac dysfunction in obesity. In the obese Wistar rats, the up - regulation of the gene expression of proteins in the left ventricles associated with calcium transport, SERCA2a, ryanodine receptor (RyR2) and phospholamban, were observed ${ }^{[51]}$. While the gene expression of proteins related to sarcolemmal calcium transport, L-type calcium channel (Cacna1c) and sarcolemmal sodium / calcium exchanger, were unchanged. In the hypertrophied rat heart with pressure overload, diastolic dysfunction is an early abnormality associated with the reduced 
transcription of SERCA2 gene ${ }^{[208]}$. Those results implied that the cardiac dysfunction in obesity is associated with changes in calcium transport related genes in the SR. Meanwhile, a pathological cardiac hypertrophy with pressure and volume overload presents a myosin isoform shift from $V_{1}(\alpha-\alpha$ dimmer $)$ type to $V_{3}(\beta-\beta \text { dimmer })^{[209]}$.

2.2 Diabetic related cardiac dysfunctions and alterations in the $\beta$-adrenergic receptor signaling pathway

The term "diabetic cardiomyopathy" was first defined as ventricular dysfunction which occurs in diabetic patients in absence of coronary artery disease and hypertension ${ }^{[210,211]}$. Now it is characterized by diastolic dysfunction, with a high prevelance of $60 \%$, even in the well controlled type 2 diabetic patients ${ }^{[212,213]}$. Diastolic dysfunction is the inability to relax and undergo appropriate filling during the diastolic phase of cardiac cycle. It is usually subclinical, but may result in diastolic heart failure in the presence of near - normal systolic function ${ }^{[214]}$. The pathophysiological changes of diabetic cardiomyopathy has not been fully understood, and some factors, including metabolic disturbances, structural changes, autonomic dysfunctions, and changes in calcium handling have been proposed for the developments of diabetic cardiomyopathy ${ }^{[61,63,215]}$. The pathological processes in the diastolic dysfunction include myocardium stiffening due to cross - linking and extracellular matrix deposition, hypertrophy, and neuronal abnormalities ${ }^{[216]}$. In the clinical studies, the diastolic and systolic dysfunctions in the type 2 diabetic patients have been identified through Doppler imaging, echocardiography, radionuclide angiography and other techniques ${ }^{[217]}$. These abnormalities include decreased LV ejection fraction, decreased myocardial velocity at early diastole, abnormal relaxation during the early filling phase, prolonged isovolumic relaxation, low peak systolic and early diastolic velocity, impaired diastolic relaxation and filling, and reduced peak filling rate depending on the age, time course and the severity of diabetes mellitus ${ }^{[218-221]}$.

The mechanisms responsible for cardiac dysfunctions in type 1 diabetes mellitus are still unclarified, and several factors have been evoked, including decreased myocardial adrenoceptor density, alterations in contractile proteins, or impaired calcium cellular movements $^{[222]}$. Streptozotocin $(\mathrm{STZ})$ - induced type 1 diabetes models were observed to develop the systolic and diastolic dysfunctions as the duration of diabetes increased. From the echocardiography observations, researchers confirmed reduced rate of circumferential shortening and fractional shortening ${ }^{[223,224]}$. In the analyses from LV catheterization, the decreased LV systolic pressure and rate of pressure rise or fall during systole and diastole $( \pm$ $\mathrm{dP} / \mathrm{dt}$ ) were confirmed ${ }^{[225,226]}$. However, in our laboratory, previous study found a preserved 
systolic function in STZ induced diabetic rats as shown by the unaltered LV ejection fraction and fractional shortening during echocardiography ${ }^{[55,56]}$. Diastolic dysfunction can be observed in many kinds of type 1 diabetic animals ${ }^{[227]}$, as shown by the prolongation of isovolumic relaxation time, increased $\mathrm{E} / \mathrm{A}$ ratio, $\mathrm{E} / \mathrm{Ea}$ ratio, and LV end-diastolic pressure in diabetic rats ${ }^{[55,56,228]}$. The cardiac dysfunction observed in the STZ - induced type 1 diabetic rats has been regarded to be associated with an increased myocardial activity of inducible nitric oxide synthase (iNOS), iNOS protein levels and expression of iNOS mRNA levels ${ }^{[55,222]}$. In the type 2 diabetic animals, cardiac dysfunction also shows diverse performances ${ }^{\text {[229-231] }}$. The $o b / o b$ mice exhibit cardiac hypertrophy with mild or no impairments in systolic function $^{[232]}$. The $d b / d b(\mathrm{C} 57 \mathrm{BL} / \mathrm{Ks})$ mice exhibit cardiac hypertrophy, and marked contractile dysfunction $^{[233]}$. In $d b / d b$ mice (18 weeks of age), which exhibit severe stage of diabetes, left ventricular systolic and diastolic functions were both preserved ${ }^{[16]}$. Some studies of the Goto - Kakizaki (GK) rats also demonstrated cardiac dysfunctions, including decreased heart rate and LV ejection fraction, and the prolongation of contraction /relaxation ${ }^{[234,235]}$. Nevertheless, a previous study performed in the Goto - Kakizaki rats and using isolated heart and the isolated cardiomyocytes, showed no significant differences compared to the controls ${ }^{[236]}$.

The cardiac function observed in Zucker diabetic (type 2) fatty rats shows highly different results ${ }^{[81,237-241]}$. Basically, the characteristics and severity of cardiac dysfunction is age related. In the Zucker obese diabetic rats aged 10 weeks, researcher found the increased LV end - diastolic volume and end - systolic volume, reduced LV ejection fraction, and reduced velocity of circumferential shortening have been noted ${ }^{[81]}$. In the young Zucker obese diabetic rats (14 weeks of age), LV dysfunction has been observed with increased end diastolic and end - systolic volume, increased end - systolic wall stress, decreased LV ejection fraction and decreased velocity of circumferential shortening using echocardiography ${ }^{[81]}$. In Zucker obese diabetic rats of 19 - 20 weeks of age, either impaired, improved, or unchanged baseline cardiac functions has been observed using echocardiography or left ventricular catheterization monitor $^{[222,241-243]}$. One research performed in aged Zucker obese diabetic rats (45 weeks of age), using LV magnetic resonance imaging and invasive catherization, showed only slight impairment of LV diastolic function, whereas LV systolic function was preserved [244]. Meanwhile, no cardiomyocyte hypertrophy was found in these aged Zucker obese diabetic rats. This result was consistent with the unchanged cardiomyocyte cross - sectional area, and unaltered mRNA expressions of hypertrophic markers brain natriuretic peptide $(\mathrm{BNP})$ and $\alpha$ skeletal actin $(\alpha \mathrm{SKA})^{[244]}$. This relatively slight cardiac dysfunction markedly contrasts with the severity of diabetic status in these aged Zucker obese diabetic rats. At the 
same time, right ventricular (RV) dysfunction in Zucker obese diabetic rats was also studied, which was related to the systematic insulin sensitivity and the decreased insulin - stimulated glucose utilization in right ventricle ${ }^{[245]}$. This is consistent with the findings of impaired RV systolic function in type 2 diabetic patients ${ }^{[246,247]}$. The remodeling of ventricles in Zucker obese diabetic rats at 14 weeks of age showed left ventricular hypertrophy without ventricular dilation, whereas, in the right ventricle, dilation without hypertrophy was noted ${ }^{[245,248]}$. The changes in both ventricles and the systolic functions might result from ventricular interdependence and diabetes influences. The myocardial activity of iNOS, iNOS protein levels and nitrotyrosine (a biomarker for oxidative damage) were observed to be higher in the Zucker obese diabetic rats at 20 weeks of age, which could lead to the reduced response to $\beta$ adrenoceptor stimulation and decreased contractile function ${ }^{[222]}$. This result suggests the role of iNOS in the pathophysiological developments of type 2 diabetes.

The basic mechanisms resulting in the diabetic cardiomyopathy are still under research, including the alterations in the $\beta$-adrenergic signaling pathway. In the diabetic patient, a blunt inotropic response was found under the stimulation of dobutamine, suggesting an impairment in the cardiac $\beta$-adrenergic pathway ${ }^{[249]}$. These authors also confirmed the down - regulation of $\beta_{1}$-adrenoceptor in diabetes mellitus ${ }^{[250,251]}$. Some previous studies about diabetic rats have reported the markedly decreased positive inotropic effects of $\beta$-adrenoceptor stimulations in vivo and in vitro ${ }^{[55,252-256]}$. In Zucker obese rats associated with diabetes, $\beta$-adrenoceptor density and affinity did not change at 6 weeks of age or 10 weeks of age compared with agematched Wistar rats, but a significant higher $\beta$-adrenoceptor affinity was observed at 20 weeks of age ${ }^{[257]}$. A few studies suggested that the diabetic - related altered positive inotropic effects were associated with the down - regulation of $\beta_{1}$-adrenoceptor, $\beta_{2}$-adrenoceptor, and the up - regulation of $\beta_{3}$-adrenoceptor ${ }^{[252,258]}$. In the hearts of chronic diabetic rats, the density of $\beta_{1}$-adrenoceptor and $\beta_{3}$-adrenoceptor were decreased and increased respectively, as in heart failure $^{[259]}$. In our laboratory, previous studies have found a decreased expression of $\beta_{1}$ adrenoceptor proteins, and an increased expression of $\beta_{3}$-adrenoceptor proteins in type 1 diabetic rats ${ }^{[55,56]}$. The $d b / d b$ diabetic mice showed no significant changes of $\beta_{1}$-adrenergic receptor $^{[16]}$. While some research showed the insignificant role of the cardiac $\beta_{2}$-adrenoceptor in diabetic ras ${ }^{[55,259]}$. In our previous studies of diabetic rats, our team already found that the diastolic dysfunction developed in early and evolved diabetes in vivo, whereas the positive lusitropic effects of $\beta$-adrenoceptor stimulation were preserved in vitro ${ }^{[55,56,260,261]}$. $\beta_{3^{-}}$ adrenoceptor is involved in this impaired positive inotropic response to $\beta$-adrenergic stimulations via the endothelial $\mathrm{NOS}_{1}$ - derived NO pathway, and the positive lusitropic 
effects were preserved in spite of the effects of $\beta_{3}$-adrenoceptor in the early and evolved diabetic cardiomyopathy of rats in vivo and in vitro ${ }^{[55,56]}$. These results suggest that the influence of $\beta_{3}$-adrenoceptor on the cardiac dysfunction may vary in different stage of diabetes. At the early stage, the activation of $\beta_{3}$-adrenoceptor may convey a protective effect against the catecholamine - induced remodeling ${ }^{[124]}$.

The post- $\beta$-adrenoceptor signaling pathways in diabetic cardiomyopathy also include a variety of components and interactions. The dual coupling of $\beta_{2}$-adrenoceptor to Gs and Gi proteins can also be found in the isolated cardiomyocytes from STZ - induced type 1 diabetic rats $^{[262]}$. The early research about heart tissue membranes of two genetic obese diabetic animals (7-12 weeks of age), male obese $d b / d b$ diabetic mice and obese diabetic CBA/Ca mice, showed no differences in the response to $\beta$-adrenergic stimulation, or adenylyl cyclase activities compared to their controls respectively ${ }^{[54]}$. Meanwhile, this study did not observe any alteration of Gs protein levels or the functional reactions between Gs protein and catalytic unit of adenylyl cyclase. In the Zucker obese diabetic rats, adenylyl cyclase activity was not elevated with age, and no significant differences were observed in unstimulated or maximum forskolin stimulated activities of adenylyl cyclase between the two strains of rats ${ }^{[257]}$. This result suggests that, although the adenylyl cyclase activity had the ability to be maximally stimulated in obese diabetic rats in vitro, the attenuation of enzyme activity still exists compared with control Wistar rats ${ }^{[257]}$. Furthermore, the basal cAMP levels reduced progressively with age in obese diabetic rats, and were significantly lower at 20 weeks of age compared with control Wistar rats. However, cAMP production in response to isoproterenol in obese diabetic rats increased higher than in control rats, which suggests a defective regulation of cAMP- phosphodiesterase in obese diabetic rats ${ }^{[257]}$. However, it has been known that the increase contractile response is not just determined by the high level of $\mathrm{cAMP}^{[82]}$. As the comparison between healthy Wistar rats and STZ - induced Wistar rats, a down-regulation of $\beta$-adrenoceptor population and reduced cAMP response to $\beta$-adrenoceptor stimulation were observed ${ }^{[257,263,264]}$. One $d b / d b$ diabetic mice study showed no significant changes in the gene expression of the SERCA2a, or PKA - mediated target proteins, whereas a marked alteration in cardiac metabolic related gene expressions was observed ${ }^{[16]}$. This research shows the role of metabolic changes involving the energy conversion in diabetic cardiomyopathy, especially when cardiac workload is increased. The reduced cardiac performance, whereas increased PKA mediated phosphorylation and the endogenous PKA activity, were observed in the STZ-induced diabetic rats ${ }^{[265]}$. 
Diabetic cardiomyopathy associated with reduced contractility and impaired relaxation, results from the changes in intracellular calcium handling, including decreased expression and activity of SERCA2a, reduced NCX expression, decreased calcium release and sequestration in SR, and compromised mitochondrial calcium cycling ${ }^{[227,266]}$. There are several conflicting researches about the SERCA2a or phospholamban of diabetic rats. In previous study, our team found a decreased expression of SERCA2a and increased expression of phospholamban in STZ - induced diabetic rats at 4 weeks or 12 weeks of age, which was associated with myocardial relaxation dysfunction in vivo and iv vitro, whereas the positive lusitropic effects to the $\beta$-adrenoceptor stimulation was preserved ${ }^{[56]}$. The reduced cardiac performance, decreased SR cycling proteins (including ryanodine receptor, SERCA2a and phospholamban), and the depressed calcium uptake and release in SR, were observed in the STZ-induced diabetic rats ${ }^{[265]}$. Further insulin treatment could improve cardiac performance, SR calcium cycling proteins contents and calcium uptake / release in SR, which suggests the role of the reduced content of calcium cycling proteins in the SR dysfunction of diabetic heart. In a sucrose (SU) - fed insulin resistance rats model, normal SERCA2a protein content but impaired SERCA activity were observed ${ }^{[267]}$. Further, the slow ventricular diastolic rate and reduced SERCA2a level were observed in Otsuka - Long-Evans Tokushima Fatty (OLETF) rats $^{[268,269]}$. One previous research in the $o b / o b$ mice showed deficiency in calcium signaling, including decreased SR calcium stores and increased SERCA ${ }^{[203]}$. The cardiomyocytes of $o b / o b$ mice have increased intracellular resting calcium concentrations, prolonged intracellular calcium decay, reduced responsiveness to extracellular calcium, and reduced SERCA2a activities ${ }^{[270]}$. The cardiac calcium handling in $d b / d b$ mice exhibited that calcium transient, L - type calcium current and SR calcium load were all reduced ${ }^{[233]}$. One research found that SERCA2a mRNA expression was not altered in Zucker obese diabetic rats ${ }^{[231]}$. In young Zucker obese diabetic rats at aged of 9-13 weeks, longer time to maximum contraction and relaxation in the cardiomyocytes in spite of the normal calcium flow amplitude were observed, suggesting the reduction in the electric current density though L-type calcium channels which was related to the changes of genes expression in the synthesis of myosin heavy chain, L-type calcium channel, intracellular calcium transport and regulation proteins ${ }^{[271]}$. This research also found that down - regulation of gene Atp2a2 (encoding SERCA2) was companied with the preserved SR calcium transport ${ }^{[271]}$. However, a study of Zucker obese diabetic rats at 19 weeks of age showed an increase of SERCA levels and decreased phospholamban levels. Furthermore, in the aged Zucker obese diabetic rats (30-34 weeks), researchers found unchanged amplitude and duration of myocyte shortening and 
relaxation, and prolonged duration of intracellular calcium transient ${ }^{[217]}$. This might be the consequence of the decreased L - type calcium current, even when the calcium content and transport in SR was unchanged in the Zucker obese diabetic rats. Meanwhile, the preserved contractility of cardiomyocytes might be associated with changing pattern in cardiac genes expressions, which encode calcium channel proteins, sodium / calcium exchanger protein, SR calcium transport proteins, cardiac muscle proteins and cardiac muscle contraction regulation proteins. A significant $50 \%$ reduction in the gene expression of SERCA2a was observed in Zucker obese diabetic rats at 45 weeks of age, which was in line with the diastolic dysfunction observed in severe diabetes ${ }^{[244]}$. Despite the different strains and models of animals, these results suggest the subtle changes in calcium regulation might exist prior to the marked ventricular dysfunction and /or heart failure, and be common in many disorders involving insulin resistance ${ }^{[272]}$. And in diabetes, the synthesis of $\beta$-myosin heavy chain increases and the contraction is slower ${ }^{[209]}$.

Because changes in contraction phase can correspondingly induce changes in relaxation phase, variations in contraction and relaxation need to be considered simultaneously to evaluate the changes in lusitropy under the $\beta$-adrenergic stimulation, which is named as contraction-relaxation coupling. Like our previous experiments about the LV papillary muscles, the coeffient $\mathrm{R}_{1}$ (ratio of maximum shortening velocity and maximum lengthening velocity, $\max \mathrm{Vc} /{ }_{\max } \mathrm{Vr}$ ) and the coefficient $\mathrm{R}_{2}$ (ratio of the peak of the positive force derivative at $\mathrm{L}_{\max }$ normalized by cross-sectional area and the peak of the negative force derivative at $\mathrm{L}_{\max }$ normalized by cross-sectional area, $+\mathrm{dF} \cdot \mathrm{dtmax}^{-1} \cdot \mathrm{s}^{-1} /-\mathrm{dF} \cdot \mathrm{dtmax}^{-1} \cdot \mathrm{s}^{-1}$ ) were used to measure contraction - relaxation coupling ${ }^{[55,56,73,253,273,274]}$. $\mathrm{R}_{1}$ reflects the contraction relaxation coupling and thus lusitropy under low load, which tests sarcoplasmic reticulum uptake calcium function in rat myocardium. $\mathrm{R}_{2}$ reflects the contraction - relaxation coupling and thus lusitropy under high load, which indirectly reflects the myofilament calcium sensitivity ${ }^{[275-277]}$. A decrease in $\mathrm{R}_{1}$ or $\mathrm{R}_{2}$ demonstrates a positive lusitropic effect. In diabetic rats, $R_{1}$ was preserved in response to $\beta$-adrenergic stimulation under isotonic condition because of the significant prolonging duration of contraction ${ }^{[56,253,274]}$. Under low load, the SR plays a major role in the regulation of relaxation. So the preservation of $R_{1}$ under $\beta$ adrenergic stimulation suggested the preservation of SR function of heart in diabetic rats. $\mathrm{R}_{2}$ did not change in response to $\beta$-adrenergic stimulation under isometric condition, suggesting the lack of alterations in myofilament calcium sensitivity in diabetic rats, compared with control rats ${ }^{[56,274]}$. It was also confirmed that myofilament sensitivity to calcium was unaltered in Zucker obese diabetic rats at ages of 9 to 13 weeks ${ }^{[271]}$. And further, a previous study about 
type 2 diabetic Goto - Kakizaki rats at ages of 18 months suggested the alteration of myofilament calcium sensitivity during the later stages of diabetes ${ }^{[234]}$.

\section{Animal models of obesity, diabetes mellitus and metabolic syndrome}

Metabolic syndrome is a group of health conditions caused by genetic and environmental factors. The main pathophysiological change of metabolic syndrome is insulin resistance, which can induce many syndromes ultimately related to the cardiovascular disease and coronary disease $^{[278]}$. For the clinical diagnosis of the metabolic syndrome, the U.S. National Health Institute made the definition as the following three or more risk factors: abdominal obesity, high triglyceride, low high density lipoprotein (HDL), high fasting glucose level and hypertension $^{[279]}$. It is a challenge to find a completely adequate animal model of metabolic syndrome to represent the different kinds of syndromes in patients with metabolic syndrome. Meanwhile, using animal models for the research about cardiomyopathy, there still exist some questions. For example, the length of cardiac cycle in mice is only one tenth of that in human, so differences certainly exist in some expression of ion channel and contractile protein isoforms ${ }^{[227]}$. However, there are still some strains of animals, especially rats, exhibiting the characteristics similar to most of metabolic syndrome patients ${ }^{[278]}$. Rodent animal models of obesity, insulin resistance and type 2 diabetes mellitus have been identified to exhibit LV hypertrophy, diastolic dysfunction, increased cardiac fatty acid uptake and utilization, reduced cardiac efficiency, impaired mitochondrial energetic, increased myocardial lipid storage, and impaired calcium handling ${ }^{[63,280,281]}$. These animals can be used to evaluate the pathophysiological changes, medications intervention and lifestyle treatments within the metabolic syndrome.

The most reliable rat strain to study metabolic syndrome seems to be Zucker obese rats, which can also be used as an obesity animal model. Some other different experimental models can be used to study metabolic syndrome for their similar abnormalities aforementioned, mainly including rats derived from spontaneous hypertensive rats (SHR) ${ }^{[278]}$. Meanwhile, some mice models, such as Psammomys obesus, leptin deficient mice (ob/ob), and apoE deficient mice, could be studied for metabolic syndrome ${ }^{[278]}$. Because of the changing lifestyle and increased intake of energy, high - fat / calorie induced obese animal models have also been used as an appropriate model to study obesity and obesity - related diseases for decades, which could reproduce the molecular, biochemical, morphological and functional alterations closely remodeling human ${ }^{[45,282-284]}$. 


\subsection{Zucker rats strains}

\subsubsection{Zucker obese rats}

In 1961, L.M. Zucker and T.F. Zucker reported an autosomal recessive mutation in fatty (fa) gene, which was shown later to be the leptin receptor gene, on chromosome 5 in rats ${ }^{[76,285]}$. This $f a$ mutation was found in a cross between Merck M strain and Sherman rats ${ }^{[76]}$. Zucker obese rats are homozygous for $f a$ allele. The presentation in these animals is a mutation in the leptin - receptor ${ }^{[286-288]}$. Leptin, generated by adipose tissue, can interact with leptin receptors in the brain, leading to the decrease in food intake and increase in energy consumption $^{[289-295]}$. The result of the leptin - receptor deficiency in Zucker obese rats is the elevation of leptin level in the circulation ${ }^{[296,297]}$. This character, together with the increase in some classical orexigenic peptides, such as neuropeptide $\mathrm{Y}$, galanin, orexins and melanin concentrating hormones, determines obesity in Zucker obese rats ${ }^{[298-301]}$.

Zucker obese rats show hyperphagia, deficient non - shivering thermogenesis and preferential deposition of energy in the adipose tissues since 17-day age ${ }^{[286,302]}$. These could influence the body weight and the proportions of body lipids in rats ${ }^{[79,303.304]}$. At the same time, some endocrinological abnormalities originated from insulin resistance can be observed as early as 3 to 4-week age in Zucker obese rats, which are dyslipidemia, mild hyperglycemia and hyperinsulinemia ${ }^{[79,303-310]}$. At 4 weeks of age, plasma insulin levels of Zucker obese rats are 4.6 times higher than that in Zucker lean rats ${ }^{[205]}$. But the plasma insulin levels will return to normal when this strain of rats live to 30 weeks of age ${ }^{[311]}$. Zucker obese rat expresses obesity with subcutaneous fat accumulation early from 5 weeks of age on ${ }^{[285]}$.

One of the primary abnormalities attributed to $f a$ gene is the increase of the activity of an enzyme, adipose tissue lipoprotein lipase. The change of its activity emerges and affects lipid filling of the adipocytes since 12 days age, long before the visual obesity in Zucker obese rats $^{[312,313]}$. Their plasma fatty acid and cholesterol levels can be ten and four times more than control animals respectively ${ }^{[278]}$. Zucker obese rats show the high low-density lipoprotein (LDL) -cholesterol and HDL-cholesterol, with the latter to be a marker of good prognosis in cardiovascular disease ${ }^{[177]}$. Moreover, the plasma cholesterol levels show different classifications between male and female obese rats ${ }^{[314]}$. For the plasma glucose, Zucker obese rats only show normal or just slightly higher levels, even though some research found the vascular alterations like diabetes mellitus in rats $^{[315]}$. The hypertensive state in Zucker obese rats still shows conflicting results ${ }^{[316-327]}$. Hypertension could be observed since 24 weeks age in Zucker obese rats ${ }^{[319]}$. The reason of the increased arterial blood pressure in Zucker obese rats is not the increased renal $\mathrm{Na}^{+}$retention, but the elevations of the proportion of adipose 
tissues, which could result in the increased production of angiotension II and reactive oxygen species $^{[319,328-330]}$. Those could promote hypertension and endothelial dysfunction.

Obesity and obesity co - morbidities may also be attributed to oxidative stress and inflammatory responses. Several factors can cause oxidative stress, such as hyperglycemia, hyperleptinemia, increased tissue lipid levels, deficiency of antioxidant defense, increased formation of free radical and chronic systematic inflammations ${ }^{[331]}$. The analysis about plasma markers of oxidative stress did not show that Zucker obese rats at 13 weeks of age are more exposed to radical species, only the coronary vessels produced higher levels of superoxide anion $^{[177]}$. Zucker obese rats develop oxidative stress, as the oxidized lipids increased in serum, urine and liver since 14 weeks of age ${ }^{[332-334]}$. The plasma antioxidant defense factors, such as glutathione peroxidase, was damaged ${ }^{[333,335]}$.

\subsubsection{Zucker obese diabetic (Zucker diabetic fatty) rats}

A phenotype of Zucker obese rats with marked diabetes was found and designated as Zucker obese diabetic rats ${ }^{[285]}$. This strain of rats is an inbred strain generated from the outbred Zucker obese rats. Zucker obese diabetic rats develop obesity, insulin resistance, hyperinsulinemia, hyperglycemia, hyperleptinemia, and hyperlipidemia. In the Zucker obese diabetic rats, obesity could be observed since 3 weeks of age and develop severely by 5 weeks of age ${ }^{[295]}$. Zucker obese diabetic rats demonstrate a higher body weight compared to the controls when they are young (9-13 weeks of age) ${ }^{[271]}$. But the research about aged Zucker obese diabetic rats at 30-34 weeks of age showed the similar body weight as the controls ${ }^{[217]}$. This is in line with the development in the diabetic patients, that is, with the age and severity of diabetes progressing and the complications worsening, diabetic individual would become more reliant on the utilization of lipids and lipid reserves to meet the metabolic requirements ${ }^{[217]}$. Meanwhile, the female Zucker obese diabetic rats are less prone to develop metabolic syndrome. ${ }^{[336,337]}$ Although having high insulin level, Zucker obese diabetic rats have been reported to become diabetics after 10 weeks of age ${ }^{[175,257]}$. The pancreatic $\beta$-cell mass in Zucker obese diabetic rats increases from 6 weeks to 16 weeks of age, and begin to decline thereafter because of apoptosis. So plasma insulin levels increase rapidly from 6 weeks to 8 weeks of age, then decline thereafter that to the levels similar to that in 6 weeks of age ${ }^{[338,339]}$. Zucker obese diabetic rats have a limit life span, and the mortality rises after 50 weeks of age ${ }^{[340,341]}$. The exact cause of the death is not clearly understood, but it has been observed that the increased mortality is associated with the increased blood urea nitrogen and renal insufficiency ${ }^{[340]}$. 
Although the model of type 2 diabetes mellitus has many similar features as in human type 2 diabetes mellitus, the presence of the leptin receptor mutation is not common in human. However, this genetic animals is considered as a reliable model of metabolic syndrome for the similarity to the development and maintenance of metabolic syndrome in human ${ }^{[175]}$. Rats developing obesity with or without the onset of type 2 diabetes have insulin resistance, which was observed in the two Zucker rat strains. Insulin resistance is associated with increased adrenergic activity, either as a cause of the increase or the consequence ${ }^{[82]}$. The increased sympathetic activity causes an increase in afterload and heart rate. And because of the greater blood volume in obese rats, the preload is also increased. Thus the cardiac output tends to increase in obese rats and obese diabetic rats. At the same time, Zucker obese diabetic rats do not develop atherosclerosis and hypertension, which are usually present in human type 2 diabetes mellitus ${ }^{[244]}$. Thus, the strain of Zucker obese diabetic rats is also a good animal model of diabetic cardiomyopathy without other co - morbidities.

\subsection{Obese spontaneous hypertensive rats}

Spontaneous hypertensive (SHR) rats, a good model to study hypertension or insulin resistance, also show hypertriglyceridemia, obesity, and hypertension ${ }^{[342,343]}$. Another different strains of SHR rats, obese SHR rats, seems to be a better model of metabolic syndrome, such as Koletsky rats, SHR/N-corpulent rats and SHR/NDmc- corpulent rats.

\subsubsection{Koletsky rats}

Koletsky rats can also be named as obese SHR or SHROB rats. This strain of rats has monogenetic obesity superimposed on hypertensive genetic background ${ }^{[317,344,345]}$. In this strain of rats, the corpulent $(c p)$ phenotype was first recognized from an autosomal recessive mutation on the Lepr gene ${ }^{[317]}$. The mutation is a Thr2349Ala transversion, causing a premature stop codon in leptin - receptors of extracellular domain. The obese SHR has two $c p$ alleles and shows leptin resistance. No matter what kind of diet, these animals show hyperphagia, hyperlipidemia (significantly high triglyceridemia and moderate elevation of cholesterol), hyperinsulinemia, hypertension, premature vascular disease and nephropathy ${ }^{[295]}$. Meanwhile, since the intake of calorie had a link with sympathetic activity, refeeding after dietary restriction to obese SHR exhibited another animal model similar to essential hypertension in obese human, which is refeeding obese $\mathrm{SHR}^{[316]}$. This strain of rats also develops increased sympathetic activity, hyperinsulinemia, insulin resistance, increase in the ventricular wall thickness and kidney diseases. They could be used to study the effects of fluctuations in the nutrition intake on the obese hypertension. 


\subsubsection{N-corpulent rats}

These rats are a substrain of Koletsky rats, characterized as non - insulin - dependent diabetes mellitus ${ }^{[295,346]}$. Obesity in this strain of rats is evident by $5-6$ weeks of age ${ }^{[347,348]}$. These animals show hyperinsulinemia, hypercholesterolemia, hyperglycemia after oral glucose load or postprandially, glycosuria and proteinuria. They develop impaired glucose regulation from 2 months of age, and the glucose tolerance improves with aging ${ }^{[349,350]}$. The hyperinsulinemic levels in this strain of rats develop from 4 weeks of age, and reach 6 times higher than controls in 5 months of age ${ }^{[347,351]}$. However, there exist sex differences in the nature of the strain of rats. Female SHR / N-cp rats are less obese than males, but serum triglyceride levels are higher than male rats, which might be associated with increased hepatic lipogenic enzyme activities ${ }^{[351]}$.

\subsubsection{NDmc-corpulent rats}

These rats are an inbred subline of SHR/N-corpulent rats and homozygous of the $c p$ gene $(c p / c p)$. These animals exihibit hyperphagia, obesity, hypertension, hyperlipidemia, insulin resistance and hyperinsulinemia, and diabetes mellitus ${ }^{[352,353]}$.

\subsubsection{Spontaneous hypertension - heart failure (SHHF) / Mcc - $c p$ rats}

$\mathrm{SHHF} / \mathrm{Mcc}-c p$ rats carry the recessive $c p$ or corpulent gene and develop obesity and the spontaneous hypertension, consequently causing cardiac dysfunction and heart failure ${ }^{[174]}$. There exist sex differences in this strain of rats. Obese male rats develop obesity, hypertension and non - insulin - dependent - diabetes mellitus, and emerge dilated cardiomyopathy since 10 to 12 months of age. Male rats would finally evolve to heart failure after 3 years old. However, obese female rats only have slight glucose tolerance abnormality and develop fatal dilated cardiomyopathy between 14 and 16 months of age ${ }^{[174,354]}$.

\subsubsection{JCR: LA - $c p$ rats}

This strain of rats carry $c p$ gene ${ }^{[174]}$. They develop obesity, dyslipidemia, insulin resistance, which are much more extreme than Zucker rats ${ }^{[355]}$. This strain of rats develop slight hyperinsulinemia at 3 weeks of age, and rapidly progress to an evident level much more severe than Zucker rats after 5 weeks of age, in which the insulin levels are higher in male rats than that in females ${ }^{[356,357]}$. At 12 weeks of age, they develop so severe insulin resistance that no insulin - mediated glucose uptake by skeletal muscle occurs. Meanwhile, they do not 
appear hyperglycemia due to islet $\beta$-cell insulin hypersecretion, so the major drawback of this rat as type 2 diabetes model is the absence of fasting hyperglycemia ${ }^{[357]}$. Elevation of triglyceride levels can be observed from 4 weeks of age exclusively attributed to the increased hepatic very low-density lipoprotein (VLDL) levels ${ }^{[358]}$. HDL levels increase in this strain of rats $^{[359]}$. But hyperlipidemia in female rats is more severe than that in males ${ }^{[356]}$. They develop early atherosclerotic lesions in the major blood vessels and occlusive coronary thrombi at later stages of disease ${ }^{[236]}$.

\subsection{Stroke - prone - spontaneous hypertensive fatty rats}

Stroke - prone spontaneous hypertensive rats (SHRSP) are an animal model developing marked hypertension and hypertension related abnormalities, including atherosclerosis, cardiac hypertrophy and nephropathy, and finally dying from stroke ${ }^{[360]}$. The stroke - prone SHR rats exhibit insulin resistance, yet no obesity, higher total cholesterol or non - esterified fatty acid (NEFA) compared with the control animals. Consequently, a new strain of rats was produced under the genetic background of stroke - prone SHR rats to be a model of metabolic syndrome ${ }^{[361]}$. This strain of rats, SHRSP fatty rats, is derived by crossing stroke - prone SHR rats of the lzumo strain to Zucker obese (fa/fa) rats and has a missense mutation in leptin receptor gene. These rats show hypertension and obesity. And the plasma leptin concentration, glucose, insulin, total cholesterol and triglyceride levels are significantly increased in these animals.

\subsection{Sterol - regulatory element - binding protein - spontaneously hypertensive rats}

This strain of rats came from a consideration that non - alcoholic fatty liver disease may be one feature of metabolic syndrome ${ }^{[362]}$. Through the transgenic over - expression of a sterol regulatory element - binding protein in SHR rats, a non - obese model of rats with hypertension, fatty liver, and several characteristics of metabolic syndrome, including hyperinsulinemia, hyperglycemia, and hypertriglyceridemia, is established.

\subsection{Wistar Ottawa Karlsburg W rats}

Wistar Ottawa Karlsburg W (WOKW) rats were produced as a new inbred strain of rats model in 1995. This strain of rats is a good animal model of metabolic syndrome because of their polygenic pathogenetic backgrounds similar to human conditions ${ }^{[363]}$. These animals show hyperphagia, and exhibit almost complete traits of metabolic syndrome with obesity, moderate hypertension, dyslipidemia, hyperinsulinemia and impaired glucose tolerance 
compared with their control animals, the dark agouti rats ${ }^{[364-366]}$. Those manifestations could emerge between 8 and 10 weeks of age in rats ${ }^{[367]}$. Meanwhile, the metabolic syndrome in WOKW also showed relations to coronary dysfunction ${ }^{[368]}$.

\subsection{Low - capacity runner rats}

This strain of rats was selectively bred according to their abilities to make a treadmill endurance running ${ }^{[369]}$. Those who could only run relatively short distance are classified as low - capacity runner (LCR) rats and selectively bred eleven generation. Then the LCR rats exhibit hypertension, endothelial dysfunction, insulin resistance, hyperinsulinemia, visceral adiposity, hypertriglyceridemia, and high NEFA.

\subsection{The streptozotocin (STZ) model}

The STZ model is the most commonly used uncontrolled type 1 diabetes model. STZ treatment intraperitoneal causes $\beta$-cell toxicity and necrosis, and finally insulin deficiency ${ }^{[370]}$. According to the recommendations from the Animal Models of Diabetic Complications Consortium (AMDCC), low - dose of STZ (50 mg/kg) administered five times consecutively can induce hyperglycemia in the rodents within 7 to 14 days after the first injection. These rodents exhibit increased serum fatty acid, triglyceride and cholesterol levels, whereas the insulin levels decrease progressively with the development of diabetes ${ }^{[371]}$. This model can be easily induced in different genetic background strains of animal and at different ages of animals. One limitation of STZ induced model is the potential extrapancreatic genotoxic effects $^{[372]}$. STZ may directly damage the cardiac contractile function through p38 mitogen activated protein (MAP) kinase - dependent oxidative stress mechanism ${ }^{[373]}$. And the disease severity in this model may be diverse, with some developing ketosis, whereas others not.

\subsection{Type 2 diabetic Goto - Kakizaki rats}

The Goto - Kakizaki (GK) rats are generated by the selective inbreeding of glucose intolerant Wistar rats ${ }^{[374,375]}$. They have impaired insulin secretion at birth $^{[81]}$. They could develop type 2 diabetes within the first few weeks of age, with mild hyperglycemia, hyperinsulinemia, impaired glucose induced insulin secretion, marked glucose intolerance, hepatic glucose overproduction and insulin resistance, but without obesity, hypertension or significant hyperlipidemia ${ }^{[374-376]}$. It has been recognized as one of the best characterized genetic animal model for the research of type 2 diabetes, without the associated influences of obesity or hypertension ${ }^{[236,377]}$. Meanwhile, type 2 diabetes mellitus is developed under a 
complex combination of environments and genetic factors. In the GK rats, at least six independent genetic loci are responsible for the deficiency in glucose and insulin metabolism $^{[374]}$. So this polygenic animal model of spontaneous type 2 diabetes mellitus is invaluable for the research of type 2 diabetes mellitus.

\subsection{DahlS.Z-Lepr ${ }^{f a} /$ Lepr $^{f a}$ (Dahl salt - sensitive (DS)/obese) rats}

Researchers established an animal model of metabolic syndrome by crossing Dahl slat sensitive (DS) rats and Zucker rats ${ }^{[378,379]}$. The DS / obese rats develop similar features to the human of metabolic syndrome, including obesity, hypertension, dyslipidemia, insulin resistance and type 2 diabetes mellitus. They also develop LV hypertrophy, LV fibrosis, LV diastolic dysfunction, cardiac oxidative stress and inflammation, and renal and liver damages ${ }^{[378,379]}$.

\subsection{The $o b / o b$ mouse}

In 1940s, diabetes was identified in $o b / o b$ mouse, which has a single autosomal recessive mutation on the obese gene ( $o b$, leptin encoding gene on chromosome 6, also known as $L e p^{o b}$ ) [227,295]. This strain of mice has a high levels of leptin mRNA in adipocytes, whereas completely lack of functional leptin ${ }^{[289,380]}$. Because of the leptin deficiency, this strain of mice develops obesity and diabetes mellitus. The strain of mice has hyperphagia, increased body fat content, decreased body temperature and energy expenditure. From 4 weeks of age, these mice on the C57BL/6 background develop moderate obesity, hyperinsulimia and impaired glucose tolerance, but not diabetes yet ${ }^{[381]}$. Since 15 weeks of age, they become severely obese. They have increased serum fatty acid and triglyceride levels dependent on the nutritional state and age. These mice die at about 14 months of age ${ }^{[382]}$. The administration of leptin can correct several diabetic manifestations in this strain of mice. The corrections of hyperinsulinamia and hyperglycemia occur before the correction of obesity ${ }^{[383-385]}$, which suggested that obesity in this model does not play an important role in the pathogenesis of diabetic manifestations.

As a hallmark of type 2 diabetes, hyperglycemia in this strain of rats can only be observed from 1 month of age, and begins to decline after 3 month of age ${ }^{[386,387]}$. This is in significant contrast to human diabetes, in which hyperglycemia progresses slowly and severely. The reasons of the difference might be the different causes of hyperglycemia in $o b / o b$ mice and human diabetes. In the former one, hyperglycemia is caused by leptin deficiency, and the persist hyperinsulinemia limits the severity of hyperglycemia, but leads to obesity ${ }^{[388,389]}$. In 
the latter one, hyperglycemia develops when compensatory hyperinsulinemia in response to insulin resistance cannot control blood glucose. The lack of leptin results in the high levels of neuropeptide $\mathrm{Y}$ and increased cortisol levels, which underlies the muscular insulin resistance $^{[390]}$. The metabolic syndrome in human develops hyperleptinemia and leptin resistance, which has been described as a key driver of obesity - related cardiovascular dysfunction $^{[391]}$. Thus, this strain of mice could be a good model for studying the effects of leptin resistance in human obesity ${ }^{[227]}$.

\subsection{The $d b / d b$ mouse}

The $d b / d b$ mice have a single autosomal recessive mutation in the leptin receptor gene on chromosome $4\left(\right.$ Lepr $\left.^{d b}\right)$, resulting in the abnormal mRNA splicing and subsequent production of a non - functional $\mathrm{Ob}-\mathrm{Rb}$ protein ${ }^{[227,295]}$ The deficiency of leptin receptor causes the over production of extracellular leptin, whereas lack of intracellular leptin action through $\mathrm{Ob}-\mathrm{Rb}$. This strain of mice develops severe type 2 diabetes since 8 weeks of age and obesity equal to $o b / o b$ mice ${ }^{[381]}$. These mice have early hyperinsulinemia and increased serum fatty acid and triglyceride levels ${ }^{[381,392]}$. The leptin receptor - deficient $\mathrm{db} / \mathrm{db}$ mice develop hyperglycemia since 2 months of age, but not all of them develop hyperglycemia ${ }^{[393]}$. At 10 weeks of age, their fasting blood glucose levels can reach about 4 times to that of the control mice ${ }^{[394]}$. Plasma insulin begins to rise at $10-14$ days and reaches to peak at 3 months of age ${ }^{[394,395]}$. This severe hyperinsulinemia is in contrast to the moderate conditions in human type 2 diabetes. And this strain of mice dies at about 10 months of age, with female living longer ${ }^{[396]}$. Insulin receptor tyrosine kinase is involved in the cellular insulin signaling process, which is markedly reduced in the muscle and liver of type 2 diabetes patients and associated with the developing of insulin resistance ${ }^{[397-399]}$. However, in the $d b / d b$ mice, the activity of the enzyme in muscle is not altered ${ }^{[400]}$. Furthermore, the $d b / d b$ mice do not develop atherosclerotic lesions despite the presences of obesity, hyperlipidemia, advanced kidney disease and cardiomyopathy ${ }^{[216]}$. 


\section{Chapter Three}

\section{Experimental Studies}

We performed two experiments about $\beta$-adrenoceptor stimulation pathway in different pathophysiological status. The first experiment is to explore the role of multidrug resistance protein 4 (MRP4) in the regulation of intracellular cyclic adenosine monophosphate (cAMP) concentration and the influence on $\beta$-adrenergic dysfunction in the senescent rat (24-month age) heart. We observed the echocardiography and isolated cardiomyocyte under baseline and stimulation of isoproterenol, along with or without the pretreatment by MK571, a specific MRP4 inhibitor. MRP4 was quantified in left ventricular homogenates by Western blotting. We confirmed that the MRP4 overexpression contributes to the decrease of positive inotropic response to $\beta$-adrenoceptor stimulation in the senescent heart. The second experiment is to compare the $\beta$-adrenoceptor signaling pathway in Zucker lean, Zucker obese, and Zucker obese diabetic rats, the reliable rat models of metabolic syndrome. The effects of $\beta$ adrenoceptor stimulation were investigated with echocardiography (baseline and isoproterenol stimulation) and in isolated left ventricular papillary muscles (baseline, and stimulations under isoproterenol, forskolin and dibutyryl cAMP). The expressions of $\beta_{1^{-}}, \beta_{2^{-}}, \beta_{3^{-}}$ adrenoceptors and MRP4 in left ventricular muscles were detected by Western Blotting. We concluded that the positive inotropic effect of $\beta$-adrenoceptor stimulation is slightly decreased in Zucker obese rats and is more markedly decreased in Zucker obese diabetic rats. These decreases are mainly related to $\beta_{1}$ - and $\beta_{2}$-adrenoceptors down-regulation. 


\section{Study No. 1}

Overexpression of cyclic adenosine monophosphate effluent protein MRP4 induces an altered response to $\beta$-adrenergic stimulation in the senescent rat heart. Carillion A, Feldman S, Jiang C, Atassi F, Na N, Mougenot N, Besse S, Hulot JS, Riou B, Amour J. Anesthesiology, 2015, 122(2): 334-342. 


\title{
Overexpression of Cyclic Adenosine Monophosphate Effluent Protein MRP4 Induces an Altered Response to $\beta$-Adrenergic Stimulation in the Senescent Rat Heart
}

\author{
Aude Carillion, M.D., Sarah Feldman, Cheng Jiang, M.D., Fabrice Atassi, Ph.D., Na Na, M.D., \\ Nathalie Mougenot, Sophie Besse, Ph.D., Jean-Sébastien Hulot, M.D., Ph.D., Bruno Riou, M.D., Ph.D., \\ Julien Amour, M.D., Ph.D.
}

\section{ABSTRACT}

Background: In the senescent heart, the positive inotropic response to $\beta$-adrenoceptor stimulation is reduced, partly by dysregulation of $\beta 1$ - and $\beta 3$-adrenoceptors. The multidrug resistance protein 4 (MRP4) takes part in the control of intracellular cyclic adenosine monophosphate concentration by controlling its efflux but the role of MRP4 in the $\beta$-adrenergic dysfunction of the senescent heart remains unknown.

Methods: The $\beta$-adrenergic responses to isoproterenol were investigated in vivo (stress echocardiography) and in vitro (isolated cardiomyocyte by Ionoptix ${ }^{\circledR}$ with sarcomere shortening and calcium transient) in young (3 months old) and senescent (24 months old) rats pretreated or not with MK571, a specific MRP4 inhibitor. MRP4 was quantified in left ventricular homogenates by Western blotting. Data are mean \pm SD expressed as percent of baseline value.

Results: The positive inotropic effect of isoproterenol was reduced in senescent rats in vivo (left ventricular shortening fraction $120 \pm 16 \%$ vs. $158 \pm 20 \%, P<0.001, \mathrm{n}=16$ rats) and in vitro (sarcomere shortening $129 \pm 37 \%$ vs. $148 \pm 35 \%, P=0.004$, $\mathrm{n}=41$ or 43 cells) as compared to young rats. MRP4 expression increased 3.6-fold in senescent compared to young rat myocardium $(P=0.012, \mathrm{n}=8$ rats per group). In senescent rats, inhibition of MRP4 by MK571 restored the positive inotropic effect of isoproterenol in vivo $(143 \pm 11 \%, \mathrm{n}=8$ rats). In vitro in senescent cardiomyocytes pretreated with MK571, both sarcomere shortening $(161 \pm 45 \%$ vs. $129 \pm 37 \%, P=0.007, \mathrm{n}=41$ cells per group) and calcium transient amplitude $(132 \pm 25 \%$ vs. $113 \pm 27 \%, P=0.007)$ increased significantly.

Conclusion: MRP4 overexpression contributes to the reduction of the positive inotropic response to $\beta$-adrenoceptor stimulation in the senescent heart. (ANesthesiology 2015; 122:334-42)

$\mathrm{E}$ LDERLY patients are exposed to a higher mortality risk during the perioperative period. ${ }^{1}$ In the senescent heart, diastolic dysfunction ${ }^{2-4}$ and a reduced response to $\beta$-adrenoceptor stimulation are observed which may contribute to hemodynamic instability during the perioperative period. ${ }^{5}$ Down-regulation of $\beta 1$ - and $\beta 2$-adrenoceptors impair the positive inotropic effect of $\beta$-adrenergic stimulation, ${ }^{2-6}$ whereas overexpression of $\beta 3$-adrenoceptor increases nitric oxide production via nitric oxide synthase 1 , activates protein kinase $\mathrm{G}$, and then promotes the increased hydrolysis of cyclic adenosine monophosphate (cAMP) by activation of phosphodiesterases. ${ }^{3}$ However, these abnormalities only partly contribute to the alteration of the $\beta$-adrenergic pathway.

A complementary mechanism of regulation of cAMP has been recently described, the multidrug resistance protein 4 (MRP4). ${ }^{7,8}$ This channel was first described in resistance to chemotherapy as it allows malignant cells to extrude the drug and survive 9 but further studies revealed its role as transporter of cyclic nucleotides. ${ }^{10-12}$ In cardiomyocytes

\section{What We Already Know about This Topic}

- The multidrug resistance protein MRP4 plays an important role in regulation of intracellular cyclic adenosine monophosphate and $\beta$-adrenergic stimulated heart performance

- Elderly patients are at significant risk of perioperative cardiovascular compromise; however, the role of MRP4 in the senescent heart has never been investigated

What This Article Tells Us That Is New

- MRP4 is overexpressed in the senescent rat heart and is involved in the limited positive inotropic response of the senescent heart to $\beta$-adrenergic stimulation

- MRP4 may be a therapeutic target for altering the inotropic reserve of elderly patients

and vascular smooth cells, MRP4 is located at the plasma membrane level ${ }^{7}$ and extrudes cAMP. ${ }^{7-9,11}$ In vitro, inhibition of MRP4 increased intracellular cAMP level and protein kinase A activity, ${ }^{8}$ leading to a greater positive inotropic

Submitted for publication January 27, 2014. Accepted for publication September 2, 2014. From the Sorbonne Universités UPMC Univ Paris 06, UMR INSERM-UPMC 1166, Paris, France (A.C., S.F., C.J., F.A., N.M., J.-S.H., B.R., J.A.); Department of Anesthesiology and Critical Care Medicine (A.C., J.A.), Department of Emergency Medicine and Surgery (N.N., B.R.), and Department of Pharmacology (J.-S.H.), Hôpital Pitié-Salpêtrière, Assistance Publique-Hôpitaux de Paris, Paris, France; Université de Reims Champagne-Ardenne and Department of Anesthesiology and Critical Care Medicine, Robert Debré Hospital, Reims, France (A.C.); Wuhan University, Wuhan, The People's Republic of China (C.J.); UMR INSERM 902 Université Evry Val d'Essone, Evry, France (S.B.); and Université Paris-Descartes, Paris, France (S.B.). 
effect of $\beta$-adrenoceptor stimulation. In mice with genetic deletion of MRP4, an increase in cAMP has been observed in cardiomyocytes after adenylyl cyclase stimulation. ${ }^{7}$ However, the involvement of MRP4 in the senescence-induced $\beta$-adrenergic dysfunction had not yet been studied.

This study tested the hypothesis that an overexpression of MRP4 contributes to the altered response of the $\beta$-adrenoceptor stimulation in the senescent heart.

\section{Materials and Methods}

\section{Animals}

Experiments were conducted in an authorized laboratory under supervision of an authorized researcher (J. Amour, A-75-20-81). The project has been submitted to the relevant Animal Care Committee through the French Ministry of High Education and Research (Comité Régional d'Ethique en Expérimentation Animale Paris-comité 3, Paris, France). Wistar male rats have been studied in two groups: senescent rats (24 months old) and young adult rats (3 months old). Animals were purchased from Janvier (Le Genest St Isle, France) and cared according to the Guiding Principles in the Care and Use of Animals in a labeled housing place (B-75-1308) with food and water ad libitum. The heart was removed during anesthesia after the measurements of arterial blood pressure and weighed, except for the hearts used for the isolation of cardiomyocytes, in order to shorten the time of ischemia. Then, the left ventricle was carefully dissected and frozen in liquid nitrogen for Western blotting experiments.

\section{Stress Echocardiography}

Transthoracic stress echocardiography was performed on anesthetized rats under 1 to $2 \%$ isoflurane using a General Electric Vivid 7 instrument (Aulnay-sous-Bois, France) as previously described..$^{3,13,14}$ Systolic function and inotropy were studied with left ventricular shortening and ejection fractions using a modified version of Simpson's analysis on parasternal short-axis and long-axis views in $M$ mode. These variables were measured in basal conditions and under $\beta$-adrenergic stimulation using isoproterenol $\left(10 \mu \mathrm{g} \mathrm{kg}^{-1} \mathrm{~min}^{-1}\right.$, continuous intravenous administration) with or without intravenous pretreatment with MK571 $(30 \mathrm{mg} / \mathrm{kg}$, Enzo Life Sciences, Villeurbanne, France) a specific inhibitor of MRP4 ${ }^{7,15}$ or the same volume of $\mathrm{NaCl} 0.9 \%$ as control. Stress values were determined after stabilization of heart rate (HR) 6 min after each drug administration.

\section{Arterial Pressure Measurements}

In vivo, at least 2 days after the echocardiographic assessment, the rats were anesthetized using pentobarbital $(50 \mathrm{mg} / \mathrm{kg}$ intraperitonally) to measure arterial blood pressure. Pressure transducer catheter (size 2F, Millar Micro-tip catheter transducer, model SPR-407; Millar Instruments, Inc., Houston, TX), was introduced into the right carotid artery and connected to a pressure transducer (Gould Electronic, Cleveland, $\mathrm{OH}$ ). After stabilization, the arterial systolic, mean arterial pressure and diastolic pressures were recorded. From the arterial blood pressure tracings, the HR and the maximum positive values of first derivative of arterial pressure $(+\mathrm{dP} / \mathrm{dt})$ were quantified. The cardiac variables were recorded under baseline conditions and under $\beta$-adrenergic stimulation using isoproterenol $\left(10 \mu \mathrm{g} \mathrm{kg}^{-1} \mathrm{~min}^{-1}\right.$, continuous intravenous administration although the tail vein) with or without pretreatment with MK571 (intravenous administration of $30 \mathrm{mg} / \mathrm{kg} 6 \mathrm{~min}$ before through the tail vein) $)^{7,15}$ or the same volume of normal saline as a control. Stress values were determined after stabilization of HR.

\section{Measurements of Intracellular Calcium Transient and Contractile Function in Isolated Cardiomyocytes}

Ventricular cardiomyocytes were isolated from rat hearts on a Langendorf apparatus using enzymatic digestion by collagenase A (Roche Diagnostics, Meylan, France) as previously described. ${ }^{16}$ Under intravenous anesthesia (pentobarbital $65 \mathrm{mg} / \mathrm{kg}$, intraperitonally) the chest was opened, the heart was removed and washed in buffer then connected through the aorta to the Langendorf perfusion cannula. An anterograde perfusion via the coronary circulation was applied to the heart with a HEPES buffer $(117 \mathrm{mM} \mathrm{NaCl}$, $5.7 \mathrm{mM} \mathrm{KCl}, 1.5 \mathrm{mM} \mathrm{KH}_{2} \mathrm{PO}_{4}, 4.4 \mathrm{mM} \mathrm{NaHCO}_{3}, 1.7 \mathrm{mM}$ $\mathrm{MgCl}_{2}, 11.7 \mathrm{mM}$ glucose, $10 \mathrm{mM}$ creatine, $21 \mathrm{mM}$ HEPES, and $20 \mathrm{mM}$ taurine, all form Sigma-Aldrich, L'Isle d'Abeau Chesnes, Saint-Quentin Fallavier, France) bubbled with oxygen and maintained at $37^{\circ} \mathrm{C}$ and $\mathrm{pH} 7.40$. The cardiac digestion was performed by the perfusion of the same buffer with collagenase A (1.2 to $1.4 \mathrm{mg} / \mathrm{ml}), 100 \mu \mathrm{M}$ EGTA and $240 \mu \mathrm{M} \mathrm{CaCl}_{2}$ (both from Sigma-Aldrich). After 60 to $80 \mathrm{~min}$, the heart was removed and the atria excised. A careful mechanical dissection completed the digestion. The cells were filtered and resuspended in the native calcium-free buffer with bovine serum albumin (Sigma-Aldrich). Calcium was progressively added to the suspension to reach an extracellular calcium concentration of $0.5 \mathrm{mM}$. Freshly isolated cardiomyocytes were used in the same day. The ventricular cardiomyocytes were loaded for $20 \mathrm{~min}$ at room temperature with Fura2-AM $(1 \mu \mathrm{M}$, Molecular Probes, Invitrogen, Saint-Aubin, France) and then resuspended in the HEPES buffer with $0.5 \mathrm{mM}$ calcium. Contractility and calcium transient of the cardiomyocytes were assessed on each cell with a Ionoptix ${ }^{\circledR}$ platform (Ionoptix Corporation, Milton, MA). ${ }^{17}$ Only rod-shaped cardiomyocytes with sharp edges were studied. Cardiomyocytes with spontaneous contraction or sarcolemal blebs were avoided. Myocytes were electrically stimulated at $1 \mathrm{~Hz}$ and $8 \mathrm{~V}$ in a room at $25^{\circ} \mathrm{C}$. The contractile properties of the cardiac myocytes were analyzed with the IonWizard ${ }^{\circledR}$ software (Ionoptix ${ }^{\circledR}$, Ionoptix Corporation) from the trace of the sarcomere shortening by peak shortening (PS), time to PS normalized to PS and the maximum shortening velocity $(-\mathrm{dL} / \mathrm{dt})$ for the shortening phase and time to $90 \%$ relengthening and the maximum relengthening velocity $(+\mathrm{dL} / \mathrm{dt})$ for the relengthening phase were recorded. 
Myocytes were alternatively exposed to a light of 340 and $380 \mathrm{~nm}$ by the Fluorescence System Interface with Hyperswitch (Ionoptix Corporation). Fura2-AM is a fluorescent dye whose emission wavelength depends on the calcium concentration and the ratio $340 / 380$ of fluorescence of the Fura2-AM is correlated to the intracellular calcium level. ${ }^{17}$ The changes in Fura2-fluorescence intensity (FFI) reflect the variation of the intracellular calcium level during the cardiac cycle, also known as calcium transient. The calcium transient was described in our study by the $\Delta F F I$, amplitude of the FFI increase after twitch (peak FFI-baseline FFI) and the time course of the fluorescence constant decay (tau). The inhibition of MRP4 was provided by a pretreatment with MK571 $\left(10^{-7} \mathrm{M}\right)$. The contraction and fluorescence ratio of the cardiomyocyte were recorded continuously at basal condition and after adjunction of isoproterenol $\left(10^{-6} \mathrm{M}\right)$. Since calibration was not performed, only percent changes with isoproterenol and baseline values of $\Delta F F I$ could be compared between groups. Stress values were evaluated at maximal cell contraction. As MK571 could also inhibit leukotrienes receptors CysLT1 and CysLT2 and thus influence the response to $\beta$-adrenoceptor stimulation, we performed additional experimentation in isolated cardiomyocytes using BAY-u9773 (10-7 M) (Cayman Chemical Bertin Pharma, Montigny-le-Bretonneux, France), a specific inhibitor of CysLT1 and CysLT2 receptors. ${ }^{18}$ Then, $\beta$-adrenoceptor stimulation was performed as described in the experiment with MK571.

\section{Western Blotting}

The left ventricles were removed from anesthetized rats and frozen in liquid nitrogen. The samples were homogenated in a lysis buffer $(50 \mathrm{mM}$ Tris $\mathrm{pH} 7.5,150 \mathrm{mM} \mathrm{NaCl}, 2 \mathrm{mM}$ EDTA, $1 \%$ triton, phosphatase and protease-inhibitor cocktail, Sigma-Aldrich). The protein concentration was determined by the Bradford protein assay (Bio-Rad, Marnes-la Coquette, France). After denaturation in Laemmli buffer, $60 \mu \mathrm{g}$ of total protein extract was loaded in each lane for separation in a sodium dodecyl sulfate $9 \%$ polyacrylamide electrophoresis gel and transferred on a nitrocellulose membrane (Hybond, Amersham, GE Healthcare, Velizy, France). After saturation in milk, each membrane was incubated overnight at $4^{\circ} \mathrm{C}$ with primary antibodies (anti-MRP4 1/200: M4I-80 (Abcam, Paris, France), anti-CysLT1 Receptor $1 / 10,000$ or anti-CysLT2 receptor $1 / 5,000$ (both from Cayman Chemical), and anti-glyceraldehyde 3-phosphate dehydrogenase (GAPDH) 1/1,500 (ab9485, Abcam)). ${ }^{8}$ The day after, membranes were washed with a Tris-saline buffer with Tween and incubated with appropriate secondary antibody (anti-rat $1 / 2,500$, anti-rabbit $1 / 2,500$, all from Cell Signaling, Ozyme, Saint-Quentin en Yvelines, France). Relative quantification of the targeted protein was achieved by fluorescence recording on EthanDIGE reader with an $\mathrm{ECL}^{\circledR}$ detection system (GE Healthcare). MRP4 was detected at $150 \mathrm{kDa}$ and $\mathrm{GAPDH}$ at $37 \mathrm{kDa}$. MRP4 expression was quantified using Image $\mathrm{J}$ software $(\mathrm{NIH}$, Bethesda, MA) and normalized versus GAPDH expression to ensure no variation in protein gel loading. In addition, Ponceau $S$ staining was performed to confirm that GAPDH expression did not differ between young and senescent rats. Two membranes were generated and comparison was performed on ratio of MRP4/GAPDH expression normalized on the mean expression in control samples in the concerning gel.

\section{Statistical Analysis}

Data are expressed as mean $\pm \mathrm{SD}$. We used absolute values to compare baseline characteristics between young and senescent rats and delta percent changes from baseline to compare the pharmacological effects, as previously desc ribed. ${ }^{3,13,14,16,19,20}$ As a matter of fact, SD of delta percent changes represents the variation of the pharmacological effect we are measuring, whereas the SD of absolute values mainly reflects interindividual differences. Moreover, some variables were expected to significantly differ at baseline because we compared young and senescent rats. The main criteria of our study was sarcomere shortening, especially PS. Assuming a baseline value of PS of $10.7 \pm 2.3 \%$, an alpha risk of 0.05 and a beta risk of 0.20 , we determined that a sample size of at least $\mathrm{n}=35$ cells per group would enable us to detect a $15 \%$ change in PS (PASS 11 software, Statistical Solutions Ltd., Cork, Ireland). Young and senescent rats or cells were studied alternatively. Experiments could not be blinded because young and senescent rats look particularly different. For in vivo experiments, treatment was allocated before anesthesia by manual randomization. For in vitro experiments, cells were parted in aliquots. Inhibitor or suspension buffer was added after manual randomization before being examined. Means were compared using the Student $t$ test or one-way ANOVA with post hoc test Newman-Keuls. All $P$ values were two-tailed and a $P$ value of less than 0.05 was considered as significant. Statistical analysis was performed using NCSS 7.0 software (Statistical Solutions Ltd.).

\section{Results}

We studied a total of 21 young and 20 senescent rats. Two young and four senescent rats exhibited ventricular fibrillation or asystole during invasive arterial pressure measurement and were excluded from the analysis.

\section{Baseline Characteristics}

Baseline characteristics of rats are reported in table 1. As expected, senescent rats had significantly higher body weight and heart weight than young rats but the heart to body weight ratio was not significantly different between groups. Using echocardiography, HR in senescent rats was significantly lower in senescent than that in young rats. Both left ventricular shortening and ejection fractions were not significantly different between senescent and young rats. Under baseline conditions in vivo, the HR was significantly lower in senescent rats but the mean arterial pressure was 
Table 1. Characteristics of Young and Senescent Rats

\begin{tabular}{lcc} 
& & \\
& Young & Senescent \\
\hline General characteristics (no. of rats) & $(\mathrm{n}=21)$ & $(\mathrm{n}=20)$ \\
Body weight (g) & $430 \pm 60$ & $566 \pm 50^{\star}$ \\
& $(\mathrm{n}=12)$ & $(\mathrm{n}=12)$ \\
Heart weight (mg) & $872 \pm 104$ & $1,294 \pm 274^{\star}$ \\
Heart weight/body weight (mg/g) & $3.2 \pm 0.3$ & $3.1 \pm 0.5$ \\
Echocardiography (no. of rats) & $(\mathrm{n}=16)$ & $(\mathrm{n}=16)$ \\
Heart rate (beats/min) & $366 \pm 33$ & $331 \pm 13^{\star}$ \\
LV ejection fraction (\%) & $81 \pm 7$ & $80 \pm 3$ \\
LV shortening fraction (\%) & $47 \pm 6$ & $44 \pm 4$ \\
Arterial catheterization (no. of rats) & $(\mathrm{n}=16)$ & $(\mathrm{n}=16)$ \\
HR (beats/min) & $420 \pm 35$ & $355 \pm 33^{\star}$ \\
Systolic arterial pressure (mmHg) & $130 \pm 16$ & $135 \pm 19$ \\
Diastolic arterial pressure (mmHg) & $105 \pm 12$ & $105 \pm 16$ \\
Mean arterial pressure (mmHg) & $113 \pm 13$ & $115 \pm 17$ \\
+dP/dt (mmHg/s) & $2,680 \pm 501$ & $2,250 \pm 665^{\star}$ \\
Sarcomere kinetics (no. of cells) & $(\mathrm{n}=43)$ & $(\mathrm{n}=41)$ \\
PS (\%) & $10.7 \pm 2.3$ & $9.5 \pm 3.0^{\star}$ \\
TPS (ms) & $11.8 \pm 3.0$ & $20.6 \pm 9.6^{\star}$ \\
-dL/dt (mm/s) & $-3.0 \pm 0.8$ & $-2.1 \pm 1.0^{\star}$ \\
+dL/dt (mm/s) & $2.7 \pm 1.0$ & $1.7 \pm 1.1^{*}$ \\
TR90 (ms) & $261 \pm 83$ & $340 \pm 102^{*}$ \\
Calcium transient (no. of cells) & $(\mathrm{n}=43)$ & $(\mathrm{n}=41)$ \\
$\Delta F F I$ (arbitrary units) & $0.53 \pm 0.19$ & $0.37 \pm 0.27 \dagger$ \\
Tau (ms) & $124 \pm 25$ & $125 \pm 28$ \\
\hline
\end{tabular}

${ }^{\star} P<0.05$ vs. Young. †Because calibration was not performed, these values could not be compared between young and senescent rats.

$-\mathrm{dL} / \mathrm{dt}=$ maximum shortening velocity $+\mathrm{dL} / \mathrm{dt}=$ maximum relengthening velocity; $+\mathrm{dP} / \mathrm{dt}=$ first derivative of arterial pressure; $\Delta \mathrm{FFI}=$ changes in Fura-2 fluorescence intensity; HR = heart rate; $L V=$ left ventricular; PS = peak shortening; TPS = time to peak shortening; TR90 = time to $90 \%$ relengthening.

not significantly different between groups. In vitro, the basal characteristics of sarcomere shortening of the cardiomyocytes revealed a moderate alteration of contractility and relaxation parameters in the senescent cardiomyocytes. The cardiomyocytes from senescent rats displayed reduced PS and $-\mathrm{dL} / \mathrm{dt}$ and increased time to PS. The $+\mathrm{dL} / \mathrm{dt}$ was significantly lower and time to $90 \%$ relengthening was significantly higher in the senescent cardiomyocytes (table 1).

\section{Effects of MK571, a Specific Inhibitor of MRP4}

Since MK571 may also inhibit leukotrienes receptors, we verified that inhibition of CysLT1 and CysLT2 receptors by BAY-u9773 did not significantly modify the response to isoproterenol on sarcomere shortening in isolated cardiomyocytes from young rats (data not shown). Moreover, CysLT1 and CysLT2 receptors protein expression were not significantly different between young and in senescent rats $(1.0 \pm 0.7$ vs. $1.0 \pm 0.8$ arbitray units, $P=0.99$ and $1.0 \pm 0.8$ vs. $1.0 \pm 0.7$ arbitray units, $P=0.90$, respectively).

Administration of saline did not significantly modify any echocardiographic variables in the senescent or young groups. In senescent rats, administration of MK571 had no significant effect on any echocardiographic variable. In contrast, in young rats, a slight but significant increase in left ventricular shortening and ejection fractions was observed (table 2). During arterial catheterization, administration
Data are mean \pm SD.

of saline or MK571 induced no significant effect on HR, mean arterial pressure, or $\mathrm{dP} / \mathrm{dt}$ in any group. Nevertheless, in young rats during echocardiography, the isoproterenolinduced increase in HR seems to be blunted by MK571 (table 2), an effect not observed during arterial catheterization (table 2). We did not test the direct effect of MK571 on sarcomere shortening and calcium transient since isolated cells were incubated with or without MK571. Nevertheless, we did not observe any significant difference in the groups incubated with or without MK571 (table 2).

\section{Effects of MK571 on $\beta$-Adrenoceptor Stimulation}

In vivo, using echocardiography, the positive inotropic effect of $\beta$-adrenoceptor stimulation was altered in senescent rats as compared with young rats. Pretreatment with MK571 restored the positive inotropic effect in the senescent group to the level observed in young rats (fig. 1).

During arterial catheterization, the increase in HR and the decrease in mean arterial pressure induced by isoproterenol were not significantly different between senescent and young rats. Pretreatment with MK571 restored the positive inotropic effect in the senescent group to the level observed in young rats (fig. 1).

In vitro, the senescent cardiomyocytes exhibited a reduced positive inotropic response to isoproterenol compared with young cardiomyocytes (fig. 2). Pretreatment with MK571 restored the positive inotropic response of sarcomere shortening in senescent cardiomyocytes returning to the level observed in young cardiomyocytes, whereas it had no significant effect in young cardiomyocytes (table 2). Calcium transient increased with $\beta$-adrenoceptor stimulation. In senescent group, calcium transient increase was more important in cardiomyocytes pretreated with MK571 than in nonpretreated cardiomyocytes (fig. 3).

\section{Expression of MRP4}

In senescent rats, MRP4 protein expression increased 3.6fold in comparison to young rats $(P=0.012)$ (fig. 4). Using Ponceau staining, we confirmed the absence of significant variation in GAPDH expression in senescent compared to young rats $(203 \pm 9$ vs. $211 \pm 15$ arbitrary units, $P=0.70)$

\section{Discussion}

In the current study, we confirmed that the positive inotropic response to $\beta$-adrenoceptor stimulation is altered in senescent hearts. We observed that the expression of MRP4 was 3.6fold increased in left ventricle of senescent rats and inhibition of MRP4 by MK571 restored the positive inotropic effect of $\beta$-adrenoceptor stimulation in senescent rats both in vivo and in vitro. In parallel, the calcium transient was improved by MK571 pretreatment in the senescent cardiomyocytes. Consequently, these results strongly support the role of MRP4 in the altered $\beta$-adrenergic response in the senescent heart.

In vivo, our results confirm that the systolic function was preserved (left ventricular shortening and ejection 
Table 2. Comparison of the Response to $\beta$-Adrenergic Stimulation in Young and Senescent Rats with or without Pretreatment by MK571

\begin{tabular}{|c|c|c|c|c|c|c|}
\hline & Baseline & Saline & Isoproterenol & Baseline & MK & Isoproterenol \\
\hline & \multicolumn{3}{|c|}{ Young } & \multicolumn{3}{|c|}{ Young with MK571 } \\
\hline Echocardiography (no. of rats) & $(n=8)$ & & & $(n=8)$ & & \\
\hline HR (beats/min) & $361 \pm 40$ & $358 \pm 20$ & $421 \pm 30^{*}$ & $371 \pm 28$ & $358 \pm 17$ & $387 \pm 29$ \\
\hline LV ejection fraction (\%) & $79 \pm 9$ & $78 \pm 7$ & $96 \pm 3^{*}$ & $83 \pm 5$ & $87 \pm 5^{\star}$ & $97 \pm 2^{*}$ \\
\hline LV shortening fraction (\%) & $46 \pm 6$ & $45 \pm 7$ & $72 \pm 8^{*}$ & $47 \pm 5$ & $53 \pm 8^{*}$ & $71 \pm 5^{\star}$ \\
\hline Arterial catheterization (no. of rats) & $(n=6)$ & & & $(n=4)$ & & \\
\hline HR (beats/min) & $440 \pm 17$ & $450 \pm 33$ & $530 \pm 23^{*}$ & $389 \pm 33$ & $384 \pm 41$ & $525 \pm 50^{*}$ \\
\hline Mean arterial pressure $(\mathrm{mmHg})$ & $116 \pm 15$ & $110 \pm 11$ & $61 \pm 8^{*}$ & $109 \pm 11$ & $107 \pm 12$ & $60 \pm 8^{*}$ \\
\hline$+\mathrm{dP} / \mathrm{dt}(\mathrm{mmHg} / \mathrm{s})$ & $2,800 \pm 566$ & $2,600 \pm 860$ & $6,733 \pm 1,115^{\star}$ & $2,500 \pm 383$ & $2,933 \pm 787$ & $5,800 \pm 1,649^{*}$ \\
\hline Sarcomere kinetics (no. of cells) & $(n=43)$ & & & & $(n=41)$ & \\
\hline PS (\%) & $10.7 \pm 2.3$ & NA & $15.4 \pm 2.5$ & NA & $9.5 \pm 3.0$ & $15.3 \pm 2.4$ \\
\hline TPS (ms) & $11.8 \pm 3.0$ & & $6.9 \pm 1.7$ & & $20.6 \pm 9.6$ & $7.0 \pm 2.6$ \\
\hline$-\mathrm{dL} / \mathrm{dt}(\mathrm{mm} / \mathrm{s})$ & $-3.0 \pm 0.8$ & & $-5.1 \pm 1.5$ & & $-2.1 \pm 1.0$ & $-5.5 \pm 2.2$ \\
\hline$+\mathrm{dL} / \mathrm{dt}(\mathrm{mm} / \mathrm{s})$ & $2.7 \pm 1.0$ & & $3.7 \pm 1.4$ & & $1.7 \pm 1.1$ & $3.9 \pm 1.3$ \\
\hline \multirow[t]{2}{*}{ TR90 (ms) } & $261 \pm 83$ & & $214 \pm 83$ & & $340 \pm 102$ & $211 \pm 59$ \\
\hline & \multicolumn{3}{|c|}{ Senescent } & \multicolumn{3}{|c|}{ Senescent with MK571 } \\
\hline Echocardiography (no. of rats) & $(\mathrm{n}=8)$ & & & $(n=8)$ & & \\
\hline Heart rate (beats/min) & $327 \pm 13$ & $322 \pm 10$ & $333 \pm 13$ & $335 \pm 12 \dagger$ & $320 \pm 23$ & $341 \pm 21$ \\
\hline LV ejection fraction (\%) & $81 \pm 4$ & $80 \pm 3$ & $89 \pm 3$ & $78 \pm 2$ & $79 \pm 3$ & $92 \pm 3$ \\
\hline LV shortening fraction (\%) & $46 \pm 4$ & $44 \pm 3$ & $54 \pm 4$ & $42 \pm 2$ & $43 \pm 2$ & $61 \pm 5$ \\
\hline Arterial catheterization (no. of rats) & $(n=4)$ & & & $(n=4)$ & & \\
\hline HR (beats/min) & $351 \pm 30$ & $376 \pm 19$ & $424 \pm 36^{\star}$ & $360 \pm 33$ & $360 \pm 33$ & $446 \pm 19^{*}$ \\
\hline Mean arterial pressure $(\mathrm{mmHg})$ & $118 \pm 16$ & $113 \pm 28$ & $73 \pm 44^{*}$ & $112 \pm 20$ & $113 \pm 30$ & $71 \pm 13^{\star}$ \\
\hline$+\mathrm{dP} / \mathrm{dt}(\mathrm{mmHg} / \mathrm{s})$ & $1,950 \pm 661$ & $2,150 \pm 412$ & $3,070 \pm 1,429^{\star}$ & $2,550 \pm 598$ & $2,750 \pm 1,370$ & $4,900 \pm 744^{*}$ \\
\hline Sarcomere kinetics (no. of cells) & $(n=41)$ & & & & $(n=41)$ & \\
\hline PS (\%) & $9.5 \pm 3.0$ & NA & $11.7 \pm 3.3 \dagger$ & NA & $8.8 \pm 2.9$ & $13.2 \pm 2.3$ \\
\hline TPS (ms) & $20.6 \pm 9.6$ & & $14.1 \pm 5.7 \dagger$ & & $20.9 \pm 8.3$ & $11.0 \pm 3.2$ \\
\hline$-\mathrm{dL} / \mathrm{dt}(\mathrm{mm} / \mathrm{s})$ & $-2.1 \pm 1.0$ & & $-3.0 \pm 1.3 \dagger$ & & $-1.8 \pm 0.8$ & $-3.7 \pm 1.2$ \\
\hline$+\mathrm{dL} / \mathrm{dt}(\mathrm{mm} / \mathrm{s})$ & $1.7 \pm 1.1$ & & $1.9 \pm 1.1 \dagger$ & & $1.7 \pm 1.2$ & $2.6 \pm 1.5$ \\
\hline TR90 (ms) & $340 \pm 102$ & & $316 \pm 65 \dagger$ & & $316 \pm 67$ & $290 \pm 74$ \\
\hline
\end{tabular}

Data are mean \pm SD. $\triangle$ is $\%$ of baseline value.

${ }^{\star} P<0.05$ vs. baseline; $\dagger P<0.05$ vs. Young.

$-\mathrm{dL} / \mathrm{dt}=$ maximum shortening velocity; $+\mathrm{dL} / \mathrm{dt}=$ maximum relengthening velocity; $+\mathrm{dP} / \mathrm{dt}=$ first derivative of arterial pressure; $\Delta \mathrm{FFI}=\mathrm{changes}$ in Fura-2 fluorescence intensity; $H R=$ heart rate; $L V=$ left ventricular; $N A=$ not applicable; PS = peak shortening; TPS = time to peak shortening; TR90 = time to $90 \%$ relengthening.

fractions) in senescent hearts, as previously observed. ${ }^{3}$ In vitro, the cardiomyocytes extracted from senescent rats exhibited an impaired sarcomere shortening with prolonged duration of shortening (time to peak shortening) and time to $90 \%$ relengthening consistent with the literature. ${ }^{2,4}$ The positive inotropic response to $\beta$-adrenoceptor stimulation was reduced in senescent rats both in vivo and in vitro. The senescent heart displays different mechanisms to preserve itself from an excessive work such as the reduction of positive chronotropic and inotropic effects of the $\beta$-adrenergic stimulation. $\beta$-Adrenergic dysfunction may contribute to long-term saving of heart function and senescence adaptation. Unfortunately during the perioperative period, this $\beta$-adrenergic dysfunction limits cardiac output adaptation and favors hemodynamic instability in aging patients. ${ }^{3}$ Although $\beta$-blockers have been shown to improve long-term survival in patients with chronic heart failure and may be also beneficial during the perioperative period, deleterious effects have also been clearly demonstrated in the perioperative period when bleeding or postoperative complications occur. ${ }^{21}$

Several modifications in the $\beta$-adrenergic signaling pathway have been described in the senescent heart. ${ }^{2,4}$ Due to decreased expression of $\beta 1$ - and $\beta 2$-adrenoceptors, less cAMP is produced in the senescent heart after isoproterenol. The effect of direct stimulation of adenylyl cyclase is also reduced, but the effect of a cAMP analog is preserved, confirming the crucial role of intracellular cAMP level in the $\beta$-adrenergic response. ${ }^{6} \beta 3$-adrenoceptor induces the production of nitrite oxide by nitrite oxide synthase 1 that activates protein kinase $\mathrm{G}$ which increases the catabolism of cAMP by phosphodiesterase activation. ${ }^{3}$ An increase in $\beta 3$ adrenoceptor expression and activity in the senescent heart ${ }^{3}$ contributes to the reduction of the positive inotropic effect of $\beta$-adrenergic stimulation as we previously observed in the diabetic cardiomyopathy. ${ }^{13}$ 
A

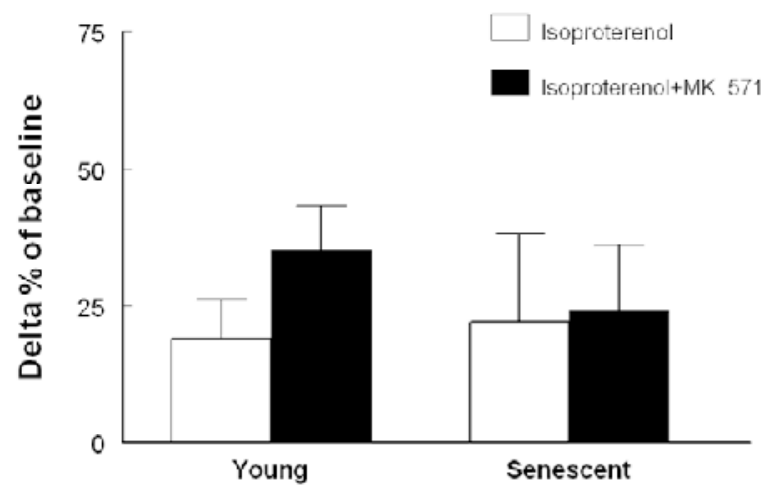

C

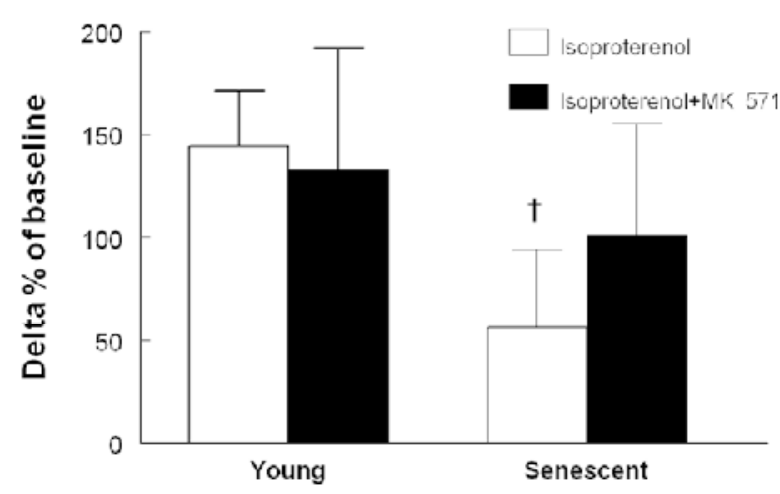

B

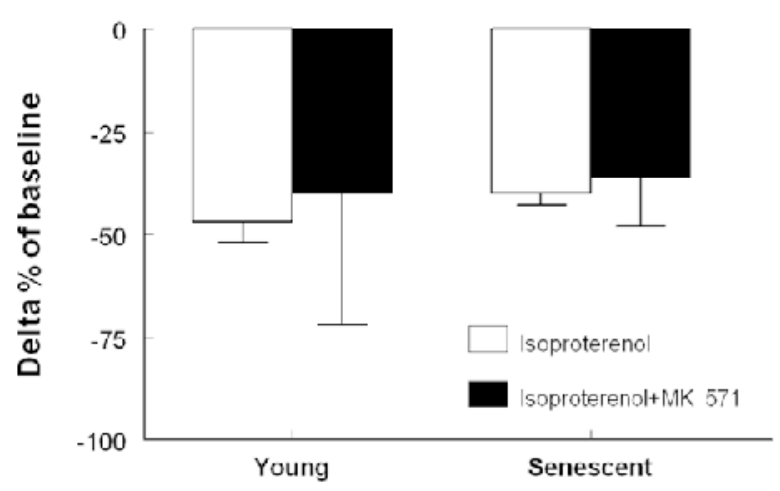

D

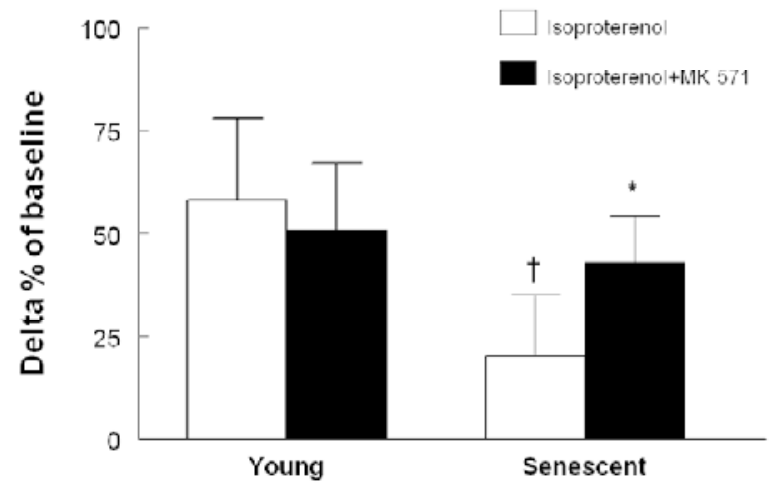

Fig. 1. Comparison of the effect of isoproterenol on heart rate (HR) $(A)$, mean arterial blood pressure (MAP) (B), positive first derivative for maximal rates of arterial pressure development $(+\mathrm{dP} / \mathrm{dt})(C)$, and left ventricular shortening fraction (LVSF) (D) in young and senescent rats with and without MK571. HR, MAP, and $+d P / d t$ were obtained during invasive catheterization $(n=6$ and 4 , respectively), and LVSF was obtained during echography ( $\mathrm{n}=8$ in each group). Data are mean \pm SD. ${ }^{*} P<0.05$ versus group without MK571; $\uparrow P<0.05$ versus Young group.

The transmembrane protein MRP4 is known as an effluent pump of cyclic nucleotides in platelets, ${ }^{22}$ hepatic, ${ }^{9}$ and renal cells. $^{23}$ MRP4 is overexpressed in the liver of female aging mice. ${ }^{24}$ In vascular smooth cells ${ }^{8,11,25}$ and cardiomyocytes, ${ }^{7}$ MRP4 is known to control the efflux of cAMP. In cultured cells, MRP4 could regulate the submembrane pool of cAMP by efflux and interaction with phosphodiesterases. ${ }^{10}$ In this context, the large MRP4 overexpression observed in senescent rats suggests a pathway for increased removal from the myocyte of cAMP that was synthesized after $\beta$-adrenoceptor stimulation. Such cAMP elimination from the cell could markedly decrease the positive inotropic effect of $\beta$-adrenergic stimulation.

MK571 has been used as a specific inhibitor of MRP4. ${ }^{7,8,11,12}$ The effect of MK571 is rapid and constant after intravenous administration. ${ }^{15,26}$ Inhibition of MRP4 by MK571 induces an elevation in cAMP intracellular concentration in vascular smooth cells $s^{8,11}$ and cardiomyocytes. ${ }^{12}$ Combined inhibition of MRP4 and phosphodiesterases induces an elevation of cAMP more important than with isolated inhibition of MRP4 suggesting that the two mechanisms may be additive. ${ }^{11}$ Inhibition of MRP4 by MK571 enhanced the chronotropic effect of isoproterenol in neonatal mice cardiomyocytes. ${ }^{12}$ In our study, MK571 had no or little effect on in vivo or in vitro contractility before $\beta$-adrenergic stimulation. This result is consistent with the fact that MK571 is not a direct agonist of $\beta$-adrenoceptors and MRP4 only affects submembrane compartment of cAMP produced after $\beta$-adrenergic stimulation. ${ }^{7}$ The elevation of cAMP after isoproterenol stimulation of isolated cardiac myocytes from 9-month-old mice is increased by silencing of MRP $4 .^{7}$ In agreement with these results, we demonstrated here that MRP 4 inhibition restored the positive inotropic effect of $\beta$-adrenoceptors stimulation both in vivo and in vitro in the senescent rats. MRP4 may extrude the cAMP produced by $\beta$-adrenoceptor stimulation by isoproterenol and may limit the increase in intracellular second messenger concentration. The intracellular calcium level closely reflects the contractility of a muscle as it is directly involved in the actin/myosin interaction and muscle shortening. The $\beta$-adrenergic stimulation induces a positive inotropic effect via cAMP production which activates the protein kinase A. Activation of protein 
A

PS

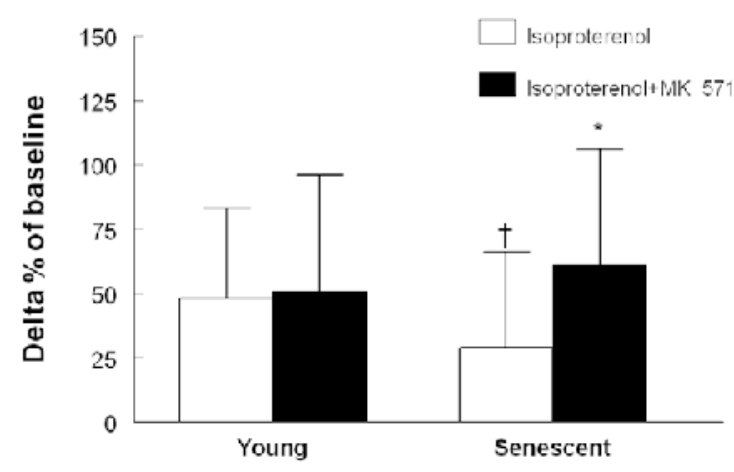

B

Time to PS

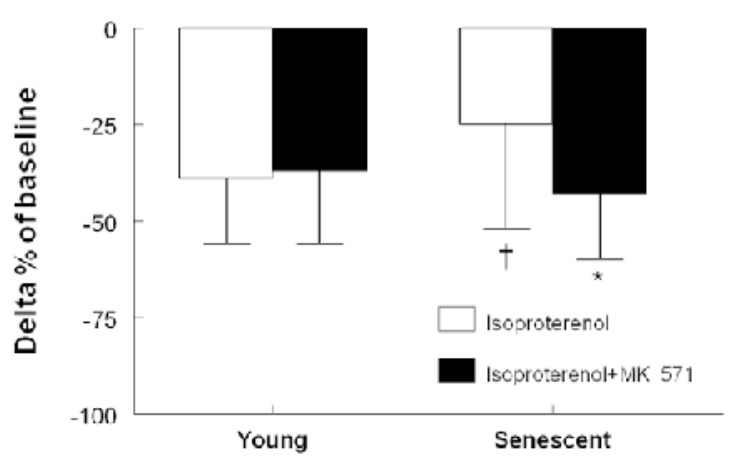

C

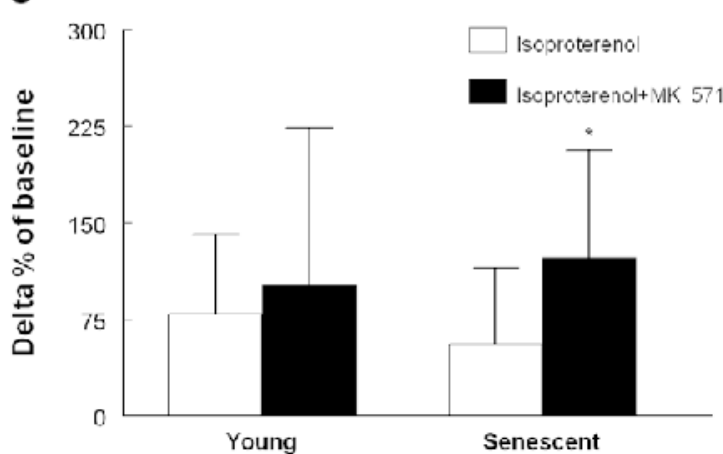

Fig. 2. Sarcomere kinetics: comparison of the effect of isoproterenol on the peak shortening (PS) amplitude $(A)$, time to PS $(B)$, and maximum velocity of shortening $(-\mathrm{dL} / \mathrm{dt})(C)$ in young and senescent rats with and without MK571 $(n=41$ to 43 cells in each group). Data are mean \pm SD. ${ }^{*} P<0.05$ versus group without MK571; $\uparrow P<0.05$ versus Young group.

kinase A increases calcium transient after phosphorylation of targeted proteins (calcium channel, ryanodine receptor, sarco-endoplasmic reticulum ATPase, and troponin). Since calcium transient was altered in senescent cardiomyocytes and since we observed an increase in calcium transient amplitude and sarcomere shortening induced by $\beta$-adrenergic stimulation after MK571 pretreatment, we can conclude that MRP4 overexpression plays a role in the altered inotropic response of $\beta$-adrenergic stimulation in the senescent heart.
A

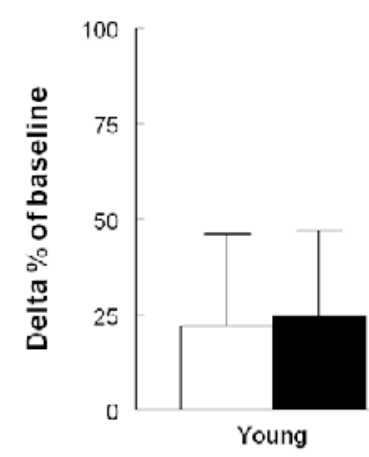

B

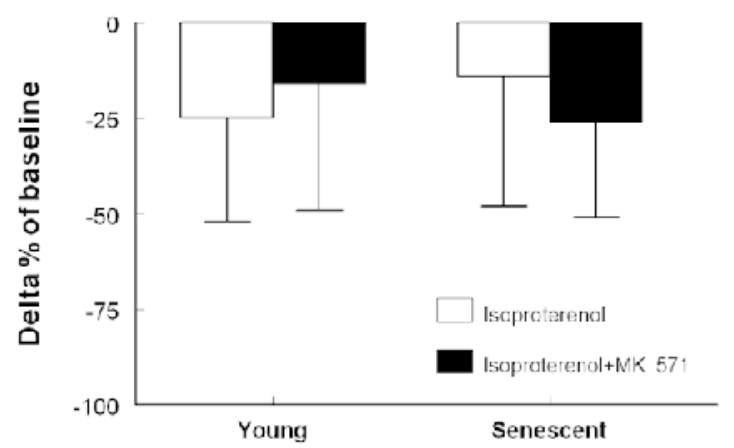

Fig. 3. Calcium transient: comparison of the effect of isoproterenol $\left(10^{-6} \mathrm{M}\right)$ on the amplitude of calcium transient (changes in Fura2-fluorescence intensity $[\Delta \mathrm{FFI}])(A)$ and time decay constant of the calcium transient (tau) $(B)$ in isolated cardiomyocytes from young and senescent rats with and without MK571 ( $n=41$ to 43 cells in each group). Data are mean \pm SD.
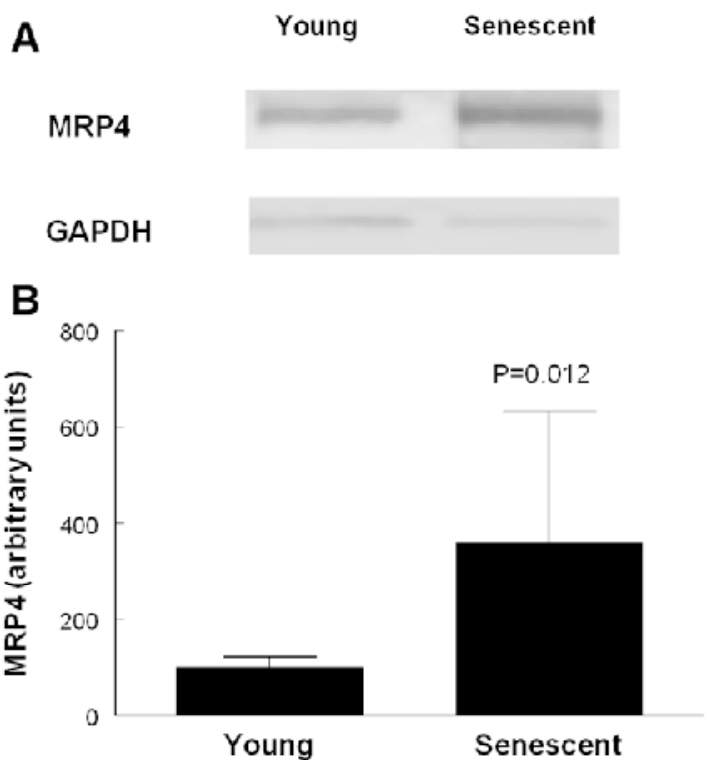

Fig. 4. Representative Western blot $(A)$ and normalized densitometric data $(B)$ showing left ventricular expression of multidrug resistance protein 4 (MRP4) compared with glyceraldehyde 3-phosphate dehydrogenase (GAPDH) in young and senescent rats ( $n=8$ in each group). Data are mean \pm SD.

Carillion et al. 
The following points have to be considered to assess the clinical relevance of our results. First, these experiments were conducted in rats and the results may not be generalized to humans as rat myocardium exhibits several differences with human myocardium, including MRP4 expression and function. ${ }^{27}$ Further experiments are mandatory to confirm the increased expression and the role of MRP4 in the human senescent heart. Second, in vivo studies require anesthetized animals. We used isoflurane inhalation for echocardiography and pentobarbital for arterial blood pressure recordings. Halogenated agents are known to interfere with $\beta$-adrenergic stimulation in different cardiomyopathies, ${ }^{19,20}$ but our results are consistent, regardless of the anesthetic technique used. Since the isoproterenol-induced increase in HR seems to be blunted by MK571 in young rats during echography (i.e., with isoflurane) but not during arterial catheterization (i.e., with pentobarbital), we cannot rule out the hypothesis that an interaction between MK571 and baroreflex activity was differentially altered by anesthetics in vivo. Third, in vitro experiments may be affected by a selection bias as cardiomyocytes surviving to cell isolation may differ from the more disabled ones present in the total heart. Fourth, we elected not to directly measure cAMP or cyclic guanosine monophophate concentrations since these messenger molecules are compartmentalized within the cell, and average cellular concentrations may not reflect critical concentrations at near relevant kinase mediators within the cell. Furthermore, MRP4 has been located in caveolae near from $\beta$-adrenoceptors and we think it could effectively act on this pool of cAMP, not necessarily on the whole cell cAMP mean concentration. ${ }^{7}$ Fifth, we used MK571 to selectively inhibit MRP4. This drug is also an inhibitor of the CysLT1 receptor for leukotrienes D4. ${ }^{15,28}$ The inhibition of CysLT1 and CysLT2 receptors by MK571 could alleviate the negative inotropic effect due to leukotrienes D4 in heart and be confusing in interpretation of our results. ${ }^{17,29}$ However, inhibition of CysLT1 and CysLT2 receptors by BAY-u9773 did not modify the response to isoproterenol in young cardiomyocytes and CysLT1 and CysLT2 receptor expressions were not significantly modified in senescent hearts. In the same manner, that we cannot rule out the possibility that the in vivo effects of MK571 could also be modulated by CysLT1 receptors in vascular smooth muscle. ${ }^{30}$ Sixth, in a therapeutic view, chronic inhibition of MRP4 may alter other organ function or may be compensated by an increase in phosphodiestereases.

In conclusion, we observed that MRP4 is overexpressed in the heart of senescent rats and plays an important role in the altered positive inotropic response to $\beta$-adrenoceptor stimulation.

\section{Acknowledgments}

The authors thank David Baker, D.M., F.R.C.A. (Department of Anesthesiology and Critical Care, Hôpital Necker-Enfants Malades, Paris, France), for reviewing the manuscript and Anne-Marie Lompré, Ph.D. (UMR INSERM 1166, Paris, France), for critical reading of the manuscript.

Dr. Carillion was the recipient of a research grant from the Fondation pour la Recherche Médicale (Paris, France; FRM grant no. DEA20090616244). Dr. Jiang was the recipient of a doctoral grant from the People's Republic of China (State Scholarship Fund by China Scholarship Council; grant no. 201206270014).

\section{Competing Interests}

The authors declare no competing interests.

\section{Correspondence}

Address correspondence to Dr. Carillion: Département d'Anesthésie-Réanimation, Groupe hospitalier PitiéSalpêtrière, 47-83 Boulevard de l'Hôpital, 75651 Paris cedex 13, France. aude.carillion@psl.aphp.fr. Information on purchasing reprints may be found at www.anesthesiology. org or on the masthead page at the beginning of this issue. Anesthesiology's articles are made freely accessible to all readers, for personal use only, 6 months from the cover date of the issue.

\section{References}

1. Gajdos C, Kile D, Hawn MT, Finlayson E, Henderson WG, Robinson TN: Advancing age and 30-day adverse outcomes after nonemergent general surgeries. J Am Geriatr Soc 2013; 61:1608-14

2. Lakatta EG: Arterial and cardiac aging: Major shareholders in cardiovascular disease enterprises: Part III: Cellular and molecular clues to heart and arterial aging. Circulation 2003; 107:490-7

3. Birenbaum A, Tesse A, Loyer X, Michelet P, Andriantsitohaina R, Heymes C, Riou B, Amour J: Involvement of beta 3-adrenoceptor in altered beta-adrenergic response in senescent heart: Role of nitric oxide synthase 1-derived nitric oxide. ANESTHESIOLOGY 2008; 109:1045-53

4. Schmidt U, del Monte F, Miyamoto MI, Matsui T, Gwathmey JK, Rosenzweig A, Hajjar RJ: Restoration of diastolic function in senescent rat hearts through adenoviral gene transfer of sarcoplasmic reticulum Ca(2+)-ATPase. Circulation 2000; 101:790-6

5. Stratton JR, Cerqueira MD, Schwartz RS, Levy WC, Veith RC, Kahn SE, Abrass IB: Differences in cardiovascular responses to isoproterenol in relation to age and exercise training in healthy men. Circulation 1992; 86:504-12

6. Xiao RP, Tomhave ED, Wang DJ, Ji X, Boluyt MO, Cheng $\mathrm{H}$, Lakatta EG, Koch WJ: Age-associated reductions in cardiac beta1- and beta2-adrenergic responses without changes in inhibitory $\mathrm{G}$ proteins or receptor kinases. J Clin Invest 1998; 101:1273-82

7. Sassi Y, Abi-Gerges A, Fauconnier J, Mougenot N, Reiken S, Haghighi K, Kranias EG, Marks AR, Lacampagne A, Engelhardt S, Hatem SN, Lompre AM, Hulot JS: Regulation of cAMP homeostasis by the efflux protein MRP 4 in cardiac myocytes. FASEB J 2012; 26:1009-17

8. Hara Y, Sassi Y, Guibert C, Gambaryan N, Dorfmüller P, Eddahibi S, Lompré AM, Humbert M, Hulot JS: Inhibition of MRP4 prevents and reverses pulmonary hypertension in mice. J Clin Invest 2011; 121:2888-97

9. Sampath J, Adachi M, Hatse S, Naesens L, Balzarini J, Flatley RM, Matherly LH, Schuetz JD: Role of MRP4 and MRP5 in biology and chemotherapy. AAPS PharmSci 2002; 4:E14

10. Xie M, Rich TC, Scheitrum C, Conti M, Richter W: Inactivation of multidrug resistance proteins disrupts both cellular extrusion and intracellular degradation of cAMP. Mol Pharmacol 2011; 80:281-93 
11. Sassi Y, Lipskaia L, Vandecasteele G, Nikolaev VO, Hatem SN Cohen Aubart F, Russel FG, Mougenot N, Vrignaud C, Lechat P, Lompré AM, Hulot JS: Multidrug resistance-associated protein 4 regulates cAMP-dependent signaling pathways and controls human and rat SMC proliferation. J Clin Invest 2008 118:2747-57

12. Sellers ZM, Naren AP, Xiang Y, Best PM: MRP4 and CFTR in the regulation of cAMP and $\beta$-adrenergic contraction in cardiac myocytes. Eur J Pharmacol 2012; 681:80-7

13. Amour J, Loyer X, Le Guen M, Mabrouk N, David JS, Camors E, Carusio N, Vivien B, Andriantsitohaina R, Heymes C, Riou $\mathrm{B}$ : Altered contractile response due to increased beta3-adrenoceptor stimulation in diabetic cardiomyopathy: The role of nitric oxide synthase 1-derived nitric oxide. ANESTHESIOLOGY 2007; 107:452-60

14. Amour J, Loyer X, Michelet P, Birenbaum A, Riou B, Heymes C: Preservation of the positive lusitropic effect of beta-adrenoceptors stimulation in diabetic cardiomyopathy. Anesth Analg 2008; 107:1130-8

15. Eppihimer MJ, Russell J, Anderson DC, Epstein CJ, Laroux S, Granger DN: Modulation of P-selectin expression in the postischemic intestinal microvasculature. Am J Physiol 1997; 273(6 pt 1):G1326-32

16. Biais M, Jouffroy R, Carillion A, Feldman S, Jobart-Malfait A, Riou B, Amour J: Interaction of metabolic and respiratory acidosis with $\alpha$ and $\beta$-adrenoceptor stimulation in rat myocardium. ANESTHESIOLOGY 2012; 117:1212-22

17. Ren J: Attenuated cardiac contractile responsiveness to insulin-like growth factor I in ventricular myocytes from biobreeding spontaneous diabetic rats. Cardiovasc Res 2000; 46:162-71

18. Liu P, Misurski DA, Gopalakrishnan V: Cysteinyl leukotriene-

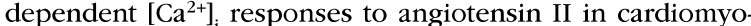
cytes. Am J Physiol Heart Circ Physiol 2003; 284:H1269-76

19. Amour J, David JS, Vivien B, Coriat P, Riou B: Interaction of halogenated anesthetics with alpha- and beta-adrenoceptor stimulations in diabetic rat myocardium. ANESTHESIOLOGY 2004; 101:1145-52

20. Vivien B, David JS, Hanouz JL, Amour J, Lecarpentier Y, Coriat P, Riou B: The paradoxical positive inotropic effect of sevoflurane in healthy and cardiomyopathic hamsters Anesth Analg 2002; 95:31-8
21. Le Manach Y, Collins GS, Ibanez C, Goarin JP, Coriat $P$, Gaudric J, Riou B, Landais P: Impact of perioperative bleeding on the protective effect of $\beta$-blockers during infrarenal aortic reconstruction. ANESTHESIOLOGY 2012; 117:1203-11

22. Jedlitschky G, Tirschmann K, Lubenow LE, Nieuwenhuis HK, Akkerman JW, Greinacher A, Kroemer HK: The nucleotide transporter MRP4 (ABCC4) is highly expressed in human platelets and present in dense granules, indicating a role in mediator storage. Blood 2004; 104:3603-10

23. Yacovino LL, Aleksunes LM: Renal efflux transporter expression in pregnant mice with type I diabetes. Toxicol Lett 2012; 211:304-11

24. Fu ZD, Csanaky IL, Klaassen CD: Effects of aging on mRNA profiles for drug-metabolizing enzymes and transporters in livers of male and female mice. Drug Metab Dispos 2012; 40:1216-25

25. Cheng D, Ren J, Jackson EK: Multidrug resistance protein 4 mediates cAMP efflux from rat preglomerular vascular smooth muscle cells. Clin Exp Pharmacol Physiol 2010; 37:205-7

26. Depré M, Margolskee DJ, Hsieh JY, Van Hecken A, Buntinx A, De Lepeleire I, Rogers JD, De Schepper PJ: Plasma drug profiles and tolerability of MK-571 (L-660,711), a leukotriene D4 receptor antagonist, in man. Eur J Clin Pharmacol 1992; 43:427-30

27. Russel FG, Koenderink JB, Masereeuw R: Multidrug resistance protein 4 (MRP4/ABCC4): A versatile efflux transporter for drugs and signalling molecules. Trends Pharmacol Sci $2008 ; 29: 200-7$

28. Wong WS, Zhu H, Liao W: Cysteinyl leukotriene receptor antagonist MK-571 alters bronchoalveolar lavage fluid proteome in a mouse asthma model. Eur J Pharmacol 2007; 575:134-41

29. Feurstein G: Leukotrienes and the cardiovascular system. Prostaglandins 1984; 27:781-92

30. Mazzetti L, Franchi-Micheli S, Nistri S, Quattrone S, Simone R, Ciuffi M, Zilletti L, Failli P: The ACh-induced contraction in rat aortas is mediated by the Cys Lt1 receptor via intracellular calcium mobilization in smooth muscle cells. $\mathrm{Br} \mathrm{J}$ Pharmacol 2003; 138:707-15 
Study No. 2

Modification of the $\beta$-adrenoceptor stimulation pathway in Zucker obese and obese diabetic rat myocardium. Jiang C, Carillion A, Na N, de Jong A, Feldman S, Lacorte JM, Bonnefont-Rousselot D, Riou B, Amour J. Crit Care Med, 2015, in press. 


\title{
Modification of the $\beta$-Adrenoceptor Stimulation Pathway in Zucker Obese and Obese Diabetic Rat Myocardium*
}

\author{
Cheng Jiang, $\mathrm{MD}^{1,2}$; Aude Carillion, $\mathrm{MD}^{1,3}$; $\mathrm{Na} \mathrm{Na}, \mathrm{MD}^{1,4}$; Audrey De Jong, MD ${ }^{3,5}$; Sarah Feldman, $\mathrm{MS}^{1}$; \\ Jean-Marc Lacorte, $\mathrm{MD}, \mathrm{PhD}^{6}$; Dominique Bonnefont-Rousselot, PharmD, $\mathrm{PhD}^{1,7,8}$; \\ Bruno Riou, $\mathrm{MD}, \mathrm{PhD}^{1,4}$; Julien Amour, $\mathrm{MD}, \mathrm{PhD}^{1,3}$
}

\begin{abstract}
Objectives: Although metabolic syndrome is associated with increased sympathetic activity that chronically stimulates $\beta$-adrenoceptors, the $\beta$-adrenoceptor signaling pathway has been poorly studied in this situation. We studied the $\beta$-adrenoceptor signaling pathway in Zucker lean, obese, and obese diabetic rats.
\end{abstract}

\section{*See also p. 1552.}

'Sorbonne Universités UPMC Univ Paris 06, UMR INSERM-UPMC 1166, IHU ICAN, Paris, France.

${ }^{2}$ Emergency Department, Zonghnan Hospital, Wuhan University, Wuhan, The People's Republic of China.

${ }^{3}$ Department of Anesthesiology and Critical Care Medicine, Hôpital PitiéSalpêtrière, Assistance Publique-Hôpitaux de Paris, Paris, France.

${ }^{4}$ Department of Emergency Medicine and Surgery, Hôpital PitiéSalpêtrière, Assistance Publique-Hôpitaux de Paris, Paris, France.

${ }^{5}$ Department of Anesthesia and Critical Care B, Hôpital Saint-Eloi, CHU de Montpellier, Montpellier, France.

${ }^{6}$ Department of Endocrinologic and Oncologic Biochemistry, Hôpital PitiéSalpêtrière, Assistance Publique-Hôpitaux de Paris, Paris, France.

${ }^{7}$ Department of Metabolic Biochemistry, Hôpital Pitié-Salpêtrière, Assistance Publique-Hôpitaux de Paris, Paris, France.

${ }^{8}$ Department of Biochemistry, Faculty of Pharmacy, Université Paris Descartes, Paris, France.

Dr. Jiang did the statistics. Drs. Jiang, Riou, and Amour conceived the study and wrote the article. Drs. Jiang, Carillion, $\mathrm{Na}$, and Feldman were involved in the experiments. Drs. Lacorte and Bonnefont-Rousselot performed the biochemical analysis. All authors read, corrected, and approved the final article. Supported solely by institutional and/or departmental sources.

Dr. Jiang was the recipient of a doctoral grant from the People's Republic of China (the State Scholarship Fund by China Scholarship Council, file No. 201206270014). Dr. Carillion received grant support from the Fondation pour le Recherche Médicale. Dr. Feldman received grant support from Bourse Pierre Viars recherche 2012 and received support for article research from the Association pour la recherche clinique et experimentale en anesthésie-réanimation de la Pitié-Salpétrière. Dr. Riou served as a board member for LFB (Les Ulis, France); provided expert testimony for ThermoFisher (Clichy, France) and Crossject (Paris, France); lectured for ThermoFisher (Clichy, France), Sanofi (Paris, France), and LFB (Les Ulis, France); and received support for travel from ThermoFisher (Clichy, France) (all unrelated to this research). The remaining authors have disclosed that they do not have any potential conflicts of interest. For information regarding this article, E-mail: bruno.riou@psl.aphp.fr

Copyright (c) 2015 by the Society of Critical Care Medicine and Wolters Kluwer Health, Inc. All Rights Reserved.

\section{DOI: 10.1097/CCM.0000000000000999}

Design: Experimental, prospective study.

Setting: University medical research laboratory.

Subjects: Adult male Zucker lean (control), obese, and obese diabetic rats.

Interventions: The effects of $\beta$-adrenoceptor stimulation were investigated in vitro in isolated left ventricular papillary muscles in control, obese, and obese diabetic rats. $\beta_{1}^{-}, \beta_{2}{ }^{-}$, and $\beta_{3}$-adrenoceptors and multidrug resistance-associated protein 4 were quantified by Western Blotting. Triglyceride, cholesterol, leptin, adiponectin, and C-peptide plasma concentrations were measured. Data are mean $\pm \mathrm{SD}$.

Measurements and Main Results: Hyperlipidemia, high leptin, and C-peptide concentrations were observed in obese and obese diabetic strains, whereas hyperglycemia occurred only in the diabetic strain. The positive inotropic effect of isoproterenol was slightly reduced in obese rats $(183 \% \pm 11 \%$ of baseline; $p=0.003 ; n=7)$ and markedly reduced in obese diabetic rats $(137 \% \pm 18 \%$ of baseline; $p<0.001$; $n=10)$ when compared with control rats $(210 \% \pm 17 \%$ of baseline; $n=9)$. $\beta_{1}$-adrenoceptors were down-regulated in obese $(-41 \%$; $p=0.02)$ and diabetic $(-54 \% ; p=0.003)$ when compared with control rats, whereas $\beta_{3}$-adrenoceptors and multidrug resistanceassociated protein expression remained unchanged. Direct stimulation of adenylate cyclase with forskolin or administration of $3^{\prime}, 5^{\prime}$-cyclic adenosine monophosphate suggests that subtle impairments also occurred beside the down-regulation of $\beta_{1}$-adrenoceptor.

Conclusions: The positive inotropic effect of $\beta$-adrenoceptor stimulation is slightly decreased in Zucker obese rats and was more markedly decreased in Zucker diabetic rats. These decreases are mainly related to $\beta_{1}$-adrenoceptor down-regulation. (Crit Care Med 2015; 43:e241-e249)

Key Words: $\beta$-adrenoceptor; cardiac muscle; catecholamines; diabetes; heart; obesity

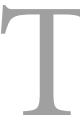
There is a worldwide increasing burden of diabetes, obesity, and related cardiovascular diseases. Metabolic syndrome (obesity, hypertension, and diabetes) is known to be associated with increased cardiovascular risk, leading to 
higher morbidity and mortality. The key elements of metabolic syndrome are obesity, impaired glucose tolerance, insulin resistance, dyslipidemia, hypertension, and increased sympathetic activity, all factors that may lead to cardiac dysfunction and ultimately congestive heart failure (1). Although obesity is associated with lower perioperative mortality (known as "obesity paradox"), patients with the metabolic syndrome are exposed to a higher mortality risk during the perioperative period (2). Such obesity paradox has also been reported in critically ill patients (3), including those with severe sepsis (4). However, several large clinical studies challenge the validity of the obesity paradox (5), and obese trauma patients are at risk of higher mortality from persistent hemorrhage (6).

In diabetic patients, diastolic dysfunction and a reduced response to $\beta$-adrenoceptor stimulation are observed, which may contribute to hemodynamic instability during critical care and the perioperative period (7). Although metabolic syndrome is associated with increased catecholamine levels and increased sympathetic activity that chronically stimulates $\beta$-adrenoceptors (8), the $\beta$-adrenoceptor signaling pathway has been poorly studied in this situation. Conflicting results have been obtained in obese swine and rabbit concerning cardiac $\beta$-adrenoceptor expression (9-11). The Zucker obese rat is considered as a reliable model of metabolic syndrome $(12,13)$. Furthermore, this model enables the separate study of obesity and obesity associated with type 2 diabetic status (14). We have previously observed alterations in $\beta$-adrenoceptor signaling pathway in type 1 diabetic (15) and senescent rats (16). Although an increase in sympathetic nervous system activation is an important mechanism for maintaining cardiac output, the positive inotropic response to $\beta$-adrenoceptor stimulation is altered in type 1 diabetic rats, in part, owing to the down-regulation of $\beta_{1}$-adrenoceptor and the up-regulation of $\beta_{3}$-adrenoceptor, which is the source of a negative inotropic effect. Active efflux transporters, namely the multidrug resistance-associated protein 4 (MRP4), also act as an independent endogenous regulator of intracellular cyclic nucleotide levels (3',5'-cyclic adenosine monophosphate, cAMP) (17) and has been recently shown to participate in $\beta$-adrenoceptor dysfunction during aging (18).

The aim of this study was to compare the effects of $\beta$-adrenoceptor stimulation in Zucker lean (as the control group), Zucker obese (also known as "Zucker fatty rats"), and Zucker obese diabetic (also known as "Zucker diabetic fatty rats") rats in vitro using isolated left ventricular papillary muscle. Our hypothesis was that metabolic syndrome is associated with some degree of $\beta$-adrenergic dysfunction, which is aggravated when associated with diabetes. We precisely assessed the effects of $\beta$-adrenergic stimulation on both contraction and relaxation and particularly focused on the following possible mechanisms involved, that is, down-regulation of $\beta_{1}$ and up-regulation of $\beta_{3}$-adrenoceptors $(15,16)$ and up-regulation of MRP4 $(17,18)$.

\section{MATERIALS AND METHODS}

Experiments were conducted in accordance with the Principles of Laboratory Animal Care (NIH publication No. 86-23, revised $1985)$ in an authorized laboratory under supervision of an authorized researcher (J.A.; A-75-20-81). The project had been approved by the relevant Animal Care Committee through the French Ministry of Higher Education and Research (Comité Régional d'Ethique en Expérimentation Animale Paris-Comité 3, Paris, France). Animals were purchased from Charles River (Saint Germain sur l'Arbresle, France) and cared in a labeled housing place (agreement number B-75-13-08) with food and water ad libitum. Animals were fed with normal rat chow containing $10 \%$ of calories from fat, $67 \%$ from carbohydrates, and $23 \%$ from proteins, $2.791 \mathrm{kcal} / \mathrm{g}$ (A04-10, SAFE, Augy, France). Three groups of 15-week-old male rats were studied: 1) Zucker lean (fa/-) rats; 2) Zucker obese (fa/fa) rats; and 3) Zucker obese $(\mathrm{fa} / \mathrm{fa})$ diabetic rats $(12,14)$. Because of the obvious morphological differences between groups, blinding was not possible.

\section{Biological Measurements}

Total cholesterol and triglyceride concentrations were determined by automated enzymatic methods $(19,20)$, and glucose concentration was assayed by hexokinase-mediated reaction (21) on a Modular P chemistry analyzer (Roche Diagnostics, Meylan, France). C-peptide was measured by quantitative enzyme-linked immunosorbent assay (ELISA) kit provided by Mercodia France (Paris, France). Rat leptin and rat total adiponectin levels were quantified using ELISA kits provided by BioVendor (Eurobio, Courtabeuf, France) and Alpco (Eurobio), respectively, and measurements were performed according to the manufacturer's instructions.

\section{Isolated Left Ventricle Papillary Muscle}

Shortly after induction of general anesthesia with pentobarbital, the heart was removed in bloc, dissected, and weighed. The left ventricular papillary muscles were carefully excised and suspended vertically in a Krebs-Henseleit bicarbonate buffer solution $\left(118 \mathrm{mM} \mathrm{NaCl}, 4.7 \mathrm{mM} \mathrm{KCl}, 1.2 \mathrm{mM} \mathrm{MgSO}_{4}, 1.1 \mathrm{mM}\right.$ $\mathrm{KH}_{2} \mathrm{PO}_{4}, 25 \mathrm{mM} \mathrm{NaHCO}_{3}, 2.5 \mathrm{mM} \mathrm{CaCl}_{2}$, and $4.5 \mathrm{mM}$ glucose) maintained at $29^{\circ} \mathrm{C}$ with a thermostatic water circulator and bubbled with $95 \%$ oxygen and $5 \% \mathrm{CO}_{2}$ as previously described (22). The papillary muscles were stimulated at $12 \mathrm{pulses} / \mathrm{min}$ for 60-minute stabilization period at the initial muscle length $\left(L_{\max }\right)$ at the apex of length-active isometric tension curve. The electromagnetic lever system has been described previously (22). All analyses were made from digital records of force and length obtained with a computer. Conventional mechanical variables at $L_{\max }$ were calculated from three twitches. The first twitch was isotonic and loaded with the preload corresponding to $L_{\max }$. The second twitch was rapidly clamped to zero load just after the electrical stimulus with a critical damping. The third twitch was fully isometric at $L_{\max }$. We determined the maximum unloaded shortening velocity $\left(V_{\max }\right)$ using the zero-load technique, and we determined maximum shortening $\left({ }_{\max } \mathrm{Vc}\right)$ and lengthening $\left({ }_{\max } \mathrm{Vr}\right)$ velocities and time to peak shortening (TPS) of the twitch with preload only. In addition, the maximum isometric active force normalized for cross-sectional area (AF), the peaks of the positive $(+\mathrm{d} F / \mathrm{d} t)$ and the negative $(-\mathrm{d} F /$ $\mathrm{d} t$ ) force derivatives at $L_{\max }$ normalized for cross-sectional area, 
and the time to peak force (TPF) from the isometric twitch were recorded. Because changes in the contraction phase induce coordinated changes in the relaxation phase, indices of contraction-relaxation coupling have been developed to study lusitropy (23). The $\mathrm{R} 1$ coefficient $\left(\mathrm{R} 1={ }_{\max } \mathrm{Vc}\right.$ max $\left._{\max } \mathrm{Vr}\right)$ studies the coupling between contraction and relaxation under low load and thus lusitropy, in a manner that is independent of inotropic changes. R1 tests sarcoplasmic reticulum (SR) calcium uptake function (23). The $\mathrm{R} 2$ coefficient $(+\mathrm{d} F / \mathrm{d} t /-\mathrm{d} F / \mathrm{d} t)$ studies the coupling between contraction and relaxation under high load and thus lusitropy, in a manner that is less dependent on inotropic changes, and reflects the myofilament calcium sensitivity (23). The cross-sectional area was calculated from the length and weight of papillary muscle, assuming a density of 1 .

Since the contractility is nearly maximum at a calcium concentration of $2.5 \mathrm{mM}$ in the rat myocardium, the extracellular calcium concentration was decreased from 2.5 to $0.5 \mathrm{mM}$ to assess the inotropic response to $\beta$-adrenoceptor stimulations, as previously described (23). A decrease in AF between $45 \%$ and $65 \%$ of baseline was required when calcium concentration was decreased. $\beta$-adrenoceptor stimulation was induced with increasing concentrations of isoproterenol $\left(10^{-8}\right.$ to $\left.10^{-4} \mathrm{M}\right)$, a nonselective $\beta$-adrenoceptor agonist, in the presence of phentolamine $\left(10^{-6} \mathrm{M}\right)$ to block $\alpha$-adrenoceptors (13). The effect of isoproterenol was expressed by the percentage of baseline value of the maximal effect of isoproterenol on $\mathrm{AF}$ and $V_{\max }\left(\mathrm{Eff}_{\max }\right)$ and the concentration of isoproterenol producing $50 \%$ of the maximal effect $\left(C_{50}\right)(23)$.

We also studied the effects of stimulation of adenylate cyclase using forskolin $\left(5 \times 10^{-5} \mathrm{M}\right)$ and the direct effect of dibutyryl cAMP $\left(5 \times 10^{-4} \mathrm{M}\right)$ as previously described (24). All drugs were purchased from Sigma-Aldrich Chimie (l'Isle d'Abeau-Chesnes, France) and added volumes never exceed $2 \%$ of the total.

\section{Immunoblotting}

The left ventricles were removed from anesthetized rats and frozen in liquid nitrogen. Total proteins were extracted in a Triton 1\% buffer with anti-phosphatase/protease inhibitor (Sigma-Aldrich). All protein concentrations were determined using Bradford reagent (BioRad, Marne-La-Coquette, France). After denaturation in Laemmli buffer, a fixed amount of mixed proteins was loaded in each lane of a sodium dodecyl sulfate polyacrylaminde gel electrophoresis (9-15\%). Proteins are separated by electrophoresis in a migration buffer and then transferred to a nitrocellulose membrane (Hybond, GE Healthcare, Vélizy, France). After saturation in milk or bovine serum albumin, each membrane was incubated overnight at $4^{\circ} \mathrm{C}$ with primary antibodies: anti-sarcoplasmic reticulum Calcium ATPase (SERCA-2a) (1:2,500, Abcam, Paris, France), antiphospholamban (1:1,000, Thermo Fisher Scientific, Rockford, IL), anti- $\beta_{1}$ adrenoceptor (1:1,000, Santa Cruz Biotechnology, Le Perray en Yvelines, France), anti- $\beta_{3}$-adrenoceptor $(1: 1,000$, Santa Cruz Biotechnology), anti- $\beta_{2}$-adrenoceptor $(1: 1,000$, Santa Cruz Biotechnology), and anti-MRP4 (multidrug-resistance protein 4) (1:200, Abcam), respectively. The following day, membranes were washed with Tris-buffered saline Tween and incubated with appropriate secondary antibody. Relative quantification of targeted protein was achieved by fluorescence recording on EthanDIGE reader with an ECL detection system (GE Healthcare, Vélizy, France). The SERCA 2a protein was detected at $110 \mathrm{kDa}$, phospholamban at $25 \mathrm{kDa}, \beta_{1}$-adrenoceptor at $65 \mathrm{kDa}$, $\beta_{2}$-adrenoceptor at $56-85 \mathrm{kDa}, \beta_{3}$-adrenoceptor at $44 \mathrm{kDa}$, and MRP4 at $150 \mathrm{kDa}$. All Western blot experiments were quantified using Image J software (NIH, Bethesda, MD) and normalized versus glyceraldehyde 3-phosphate dehydrogenase $(37 \mathrm{kDa})$, ensuring no variation in protein gel loading.

\section{Statistical Analysis}

Data are expressed as mean $\pm \mathrm{SD}$. Comparison of means was performed using the repeated-measures analysis of variance and Newman-Keuls test. All $p$ values were two-tailed, and a $p$ value of less than 0.05 was considered significant. Statistical analysis was performed using NCSS 2007 software (Statistical Solutions, Cork, Ireland). Concentration response curves were determined by fitting the data to the Hill sigmoid pharmacological model according to the following equation: Eff $_{\mathrm{o}}=\mathrm{Eff}_{\max }\left(1+\left(C_{50} C^{-1}\right)^{n}\right)^{-1}$ in which Eff $\mathrm{o}_{\mathrm{o}}$ is the observed effect, Eff ${ }_{\text {max }}$ is the maximum effect, $C_{50}$ is the concentration that results in $50 \%$ of $\mathrm{Eff}_{\text {max }}, C$ is the studied concentration, and $n$ is the Hill coefficient. Iterative nonlinear regression curve fitting was used to obtain the best fit (Matlab $1.2 \mathrm{c}$ software; The MathWorks, South Natick, MA). The main endpoint of our study was the Eff ${ }_{\text {max }}$ of the concentration-response curve of AF with isoproterenol. Assuming a value of Eff ${ }_{\max }$ of $179 \% \pm$ $15 \%$ in the control group (15), an $\alpha$ risk of 0.05 , and a $\beta$ risk of 0.20 , we determined that a sample size of at least $n=7$ papillary muscle per group would enable us to detect a $15 \%$ change in Eff $_{\text {max }}$ (PASS 11 software, Statistical Solutions).

\section{RESULTS}

\section{Characterization of the Experimental Models}

Obese rats had significantly higher body weight and heart weight than control rats, whereas obese diabetic rats did not significantly differ from control rats. The heart weight to body weight ratio was significantly decreased in obese rats mainly due to the greater increase in body weight than in heart weight (Table 1). Blood glucose levels were elevated (four times) only in obese diabetic rats. The C-peptide level, which reflects the insulin secretion, was markedly increased in obese rats while it was moderately increased in obese diabetic rats despite high blood glucose levels, suggesting $\beta$-cell dysfunction. Total cholesterol and triglycerides levels in both obese and obese diabetic rats were significantly higher than control rats. The leptin levels were significantly increased in both obese and obese diabetic rats. Adiponectin levels were not significantly modified in both obese and obese diabetic rats when compared with control rats (Table 1).

We measured the baseline characteristics of left ventricular papillary muscles in the different group of rats (Table 1). Although $V_{\max }$ was not significantly modified, a significant increase in AF was observed in obese and obese diabetic rats. Prolongation of the duration of contraction was observed in 
TABLE 1. General Characteristics of Zucker Lean (Control), Obese, and Obese Diabetic Rats

\begin{tabular}{|c|c|c|c|}
\hline Variables & Control & Obese & Obese Diabetic \\
\hline General characteristics (no. of rats) & $n=21$ & $n=21$ & $n=18$ \\
\hline Body weight (g) & $358 \pm 25$ & $498 \pm 41^{\mathrm{a}}$ & $346 \pm 23^{b}$ \\
\hline Heart weight (mg) & $921 \pm 61$ & $1,031 \pm 77^{a}$ & $888 \pm 50^{b}$ \\
\hline Heart weight/body weight (mg/g) & $0.26 \pm 0.01$ & $0.21 \pm 0.01^{\mathrm{a}}$ & $0.26 \pm 0.01^{b}$ \\
\hline Biological measurement (no. of rats) & $n=10$ & $n=10$ & $n=9$ \\
\hline Blood glucose (mmol/L) & $8.6 \pm 1.6$ & $8.6 \pm 1.2$ & $36.1 \pm 4.9^{a, b}$ \\
\hline Total cholesterol (mmol/L) & $2.58 \pm 0.22$ & $6.26 \pm 0.71^{a}$ & $4.64 \pm 0.42^{a, b}$ \\
\hline \multirow[t]{2}{*}{ Triglycerides (mmol/L) } & $1.39 \pm 0.69$ & $3.73 \pm 1.38^{a}$ & $3.00 \pm 1.59^{a}$ \\
\hline & $n=8$ & $n=9$ & $n=9$ \\
\hline Leptin $(\mu \mathrm{g} / \mathrm{L})$ & $5.2 \pm 1.9$ & $61.0 \pm 11.8^{a}$ & $10.0 \pm 1.4^{\mathrm{a}, \mathrm{b}}$ \\
\hline Adiponectin (mg/L) & $3.2 \pm 0.4$ & $3.3 \pm 0.5$ & $4.3 \pm 1.0$ \\
\hline C-peptide $(\mu \mathrm{mol} / \mathrm{L})$ & $1.29 \pm 0.51$ & $6.59 \pm 2.25^{a}$ & $1.85 \pm 0.53^{\mathrm{a}, \mathrm{b}}$ \\
\hline Mechanical properties (no. of muscles) & $n=28$ & $n=25$ & $n=24$ \\
\hline$L_{\max }(\mathrm{mm})$ & $4.2 \pm 1.5$ & $3.9 \pm 1.8$ & $3.1 \pm 1.2^{a, b}$ \\
\hline Cross-sectional area $\left(\mathrm{mm}^{-2}\right)$ & $0.55 \pm 0.13$ & $0.54 \pm 0.20$ & $0.43 \pm 0.16^{a, b}$ \\
\hline Maximal unloading isotonic shortening velocity $\left(L_{\max } / \mathrm{s}\right)$ & $2.35 \pm 0.38$ & $2.43 \pm 0.37$ & $2.41 \pm 0.50$ \\
\hline $\begin{array}{l}\text { Active force normalized per cross-sectional area during } \\
\text { isometric contraction }\left(\mathrm{mN} / \mathrm{mm}^{2}\right)\end{array}$ & $51 \pm 8$ & $59 \pm 11^{a}$ & $67 \pm 13^{a, b}$ \\
\hline Time to peak shortening (ms) & $164 \pm 9$ & $173 \pm 12^{\mathrm{a}}$ & $196 \pm 13^{a, b}$ \\
\hline Time to peak force (ms) & $152 \pm 11$ & $160 \pm 13^{a}$ & $179 \pm 16^{a, b}$ \\
\hline $\begin{array}{l}\text { Ratio of maximum shortening to maximum shortening velocity } \\
\text { to maximum lengthening velocity (i.e., contraction relaxation } \\
\text { coupling under low load) }\end{array}$ & $0.55 \pm 0.10$ & $0.57 \pm 0.16$ & $0.60 \pm 0.11$ \\
\hline $\begin{array}{l}\text { Ratio of the peak of positive force derivative to the peak negative } \\
\text { force derivative (i.e., contraction relaxation coupling under low load) }\end{array}$ & $1.71 \pm 0.23$ & $1.87 \pm 0.28$ & $2.08 \pm 0.40^{\mathrm{a}}$ \\
\hline
\end{tabular}

$L_{\max }=$ initial muscle length.

${ }^{\mathrm{a}} p<0.05$ versus control group.

${ }^{\mathrm{b}} p<0.05$ between obese and obese diabetic rats.

Data are mean \pm SD.

obese and obese diabetic rats as shown by the prolongation of TPS and TPF. No significant difference was observed in contraction-relaxation coupling under low load (R1, Table 1). By contrast, contraction-relaxation coupling under high load (R2) was significantly increased in obese diabetic rats (Table 1).

\section{$\boldsymbol{\beta}$-Adrenergic Stimulation}

$\beta$-adrenoceptor stimulation induced a marked positive inotropic effect in control rats (Fig. 1 and Table 2). A slight decrease in this inotropic response was observed in obese rats and a marked decrease was observed in obese diabetic rats in low $\left(V_{\max }\right)$ and high (AF) loading conditions (Fig. 1 and Table 2).

In control rats, $\beta$-adrenoceptor stimulation induced a positive lusitropic effect under low load (R1) but not under high load (R2) (Table 2). Comparable effects were observed in control, obese, and obese diabetic rats, and there was no significant difference between strains (Table 2).
Figure 2 shows the comparison of the stimulation of $\beta$-adrenoceptors by isoproterenol, the stimulation of adenylate cyclase by forskolin, or direct stimulation by dibutyryl cAMP. The results obtained with forskolin were consistent with those obtained with isoproterenol, indicating a moderate impairment in the positive inotropic response in obese rats and a marked impairment in obese diabetic rats. By contrast, using dibutyryl cAMP, the degree of impairment in the positive inotropic response did not significantly differ between obese and obese diabetic rats (Fig. 2).

\section{Immunoblotting}

In agreement with the functional changes observed in the papillary muscle experiments, we found that the abundance of proteins for $\beta_{1}$-adrenoceptor were significantly reduced by $40 \%(p=0.02)$ in obese rats and 54\% $(p=0.003)$ in obese diabetic rats compared with levels measured in control rats 

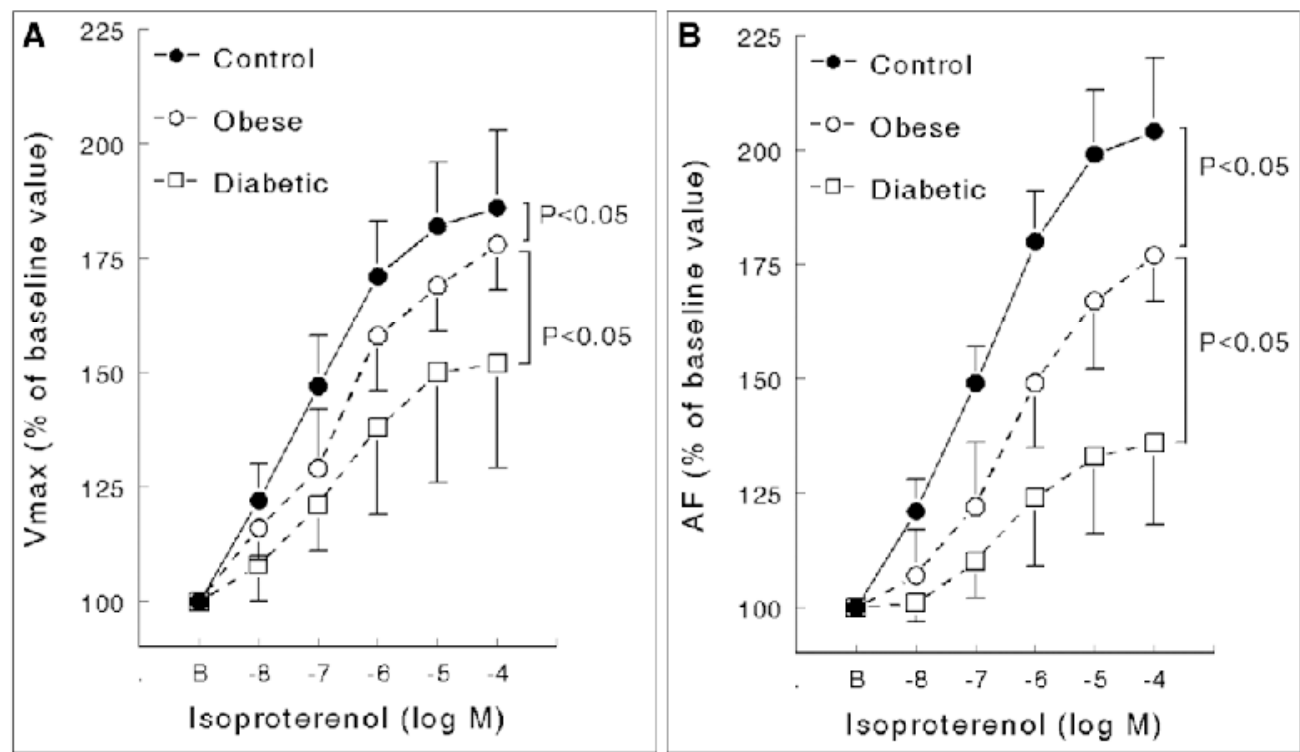

Figure 1. Inotropic response to $\beta$-adrenoceptor stimulation (isoproterenol) in control $(n=9)$, obese $(n=7)$, and obese diabetic $(n=10)$ Zucker rats under low $(\mathbf{A})$ and high $(\mathbf{B})$ loads. Data are mean percentage of baseline value \pm SD. The $p$ values refer to the comparison of the maximum effect reported in Table 2 . AF $=$ isometric active force normalized by cross-sectional area, $V_{\max }=$ maximum shortening velocity. $p=0.03$, respectively) and in obese diabetic rats $(2.0 \pm 1.0$, $p=0.003$ and $1.3 \pm 0.8$, $p=0.02$, respectively) when compared with control rats (4.2 \pm 1.3 and $4.8 \pm 4.0$, respectively). No significant changes were noted for phospholamban $(p=0.71)($ Fig. 3D) and MRP4 $(p=0.64)$ (Fig. 3F). The abundance of SERCA 2a protein was significantly reduced by $40 \%$ $(p=0.03)$ in obese rats but not significantly modified in obese diabetic rats $(p=0.67)$ (Fig. $3 E)$. But the SERCA $2 \mathrm{a} /$ phospholamban ratio was not significantly decreased $(p=0.61)$ in obese rat $(3.5 \pm 1.8)$ and obese diabetic rats $(3.5 \pm 1.8)$ when compared with control rats $(3.9 \pm 2.5)$
(Fig. 3A). A similar decrease was observed in $\beta_{2}$-adrenoceptor protein (Fig. $3 B$ ). By contrast, $\beta_{3}$-adrenoceptors were not significantly modified in obese and obese diabetic rats $(p=0.97)$ (Fig. $3 C$ ). Consequently, the $\beta_{1} \beta_{3}$ and $\beta_{2 /} \beta_{3}$ ratios were significantly decreased in obese rats $(2.4 \pm 1.3, p=0.01$ and $1.8 \pm 1.3$,

\section{DISCUSSION}

In the present study, we observed a slight decrease in the positive inotropic effect of $\beta$-adrenoceptor stimulation in obese rats. In obese diabetic rats, the decrease was more pronounced but not as severe as that previously observed in type 1 diabetic

\section{TABLE 2. Comparison of the Inotropic Response to $\beta$-Adrenoceptor Stimulation (Isoproterenol) in Zucker Lean (Control), Obese, and Obese Diabetic Rats}

$\begin{array}{lccr}\text { Variables } & \text { Control }(\boldsymbol{n}=\mathbf{9}) & \text { Obese }(\boldsymbol{n}=\mathbf{7}) & \text { Obese Diabetic }\left(n=156 \pm 26^{\mathrm{a}, \mathrm{b}}\right. \\ \text { Maximum unloaded shortening velocity } & & & \\ \text { Eff }_{\text {max }}(\% \text { of baseline }) & 193 \pm 18 & 182 \pm 14 & 0.20 \pm 0.09 \\ C_{50}(\mu \mathrm{mol} / \mathrm{L}) & 0.05 \pm 0.05 & 0.27 \pm 0.41 & \end{array}$

Maximum isometric active force normalized by cross-sectional area
$\mathrm{Eff}_{\max }(\%$ of baseline)
$210 \pm 17$
$183 \pm 11^{\mathrm{a}}$
$137 \pm 18^{\mathrm{a}, \mathrm{b}}$
$C_{50}(\mu \mathrm{mol} / \mathrm{L})$
$0.11 \pm 0.11$
$0.53 \pm 0.63^{a}$
$0.53 \pm 0.32^{a}$

Ratio of maximum shortening velocity to maximum lengthening velocity (i.e., contraction relaxation coupling under low load)
$\mathrm{Eff}_{\max }(\%$ of baseline)
$74 \pm 8$
$73 \pm 13$
$64 \pm 10$
$C_{50}(\mu \mathrm{mol} / \mathrm{L})$
$0.15 \pm 0.30$
$0.14 \pm 0.26$
$0.39 \pm 0.48$

Ratio of the peak of positive force derivative to the peak of negative force derivative (i.e., contraction relaxation coupling under high load)
$\mathrm{Eff}_{\max }(\%$ of baseline $)$
$90 \pm 17$
$100 \pm 27$
$89 \pm 25$
$C_{50}(\mu \mathrm{mol} / \mathrm{L})$
ND
ND
ND

$\mathrm{C}_{50}=$ concentration of isoproterenol producing $50 \%$ of $\mathrm{Eff}_{\max }, \mathrm{Eff}_{\max }=$ maximum effect in percentage of baseline value, $n=$ number of papillary muscles $\mathrm{ND}=$ not determined.

${ }^{a} p<0.05$ versus control.

${ }^{\mathrm{b}} p<0.05$ between obese and obese diabetic rats.

Data are mean \pm SD.

$\mathrm{C}_{50}$ was not determined when $\mathrm{Eff}_{\max }$ was not significantly different from baseline values. 


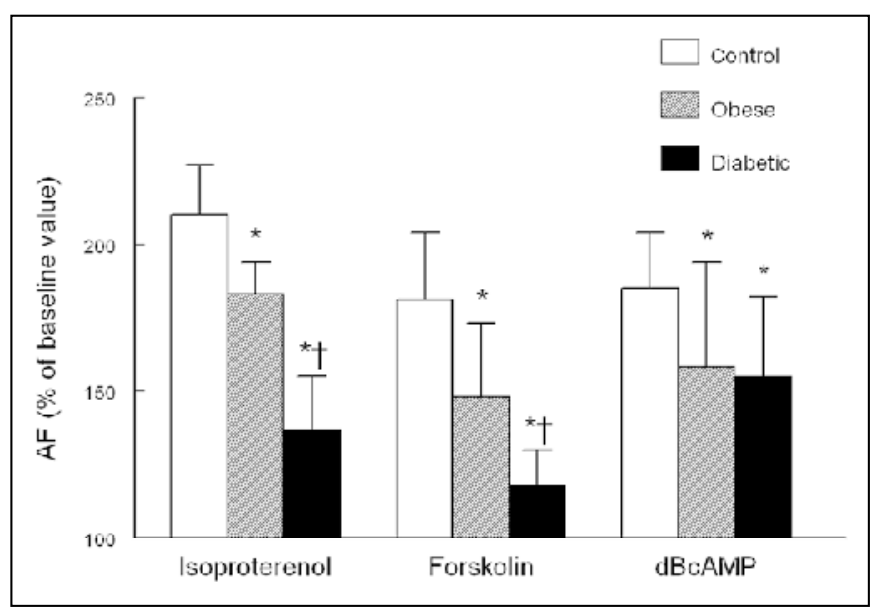

Figure 2. Comparison of the positive inotropic effects of isoproterenol $\left(10^{-4} \mathrm{M}\right)$, forskolin $\left(5 \times 10^{-5} \mathrm{M}\right)$, and dibutyryl cyclic adenosine monophosphate $\left(\mathrm{dBcAMP} ; 5 \times 10^{-4} \mathrm{M}\right)$ in left ventricular papillary muscles from Zucker control, obese, and obese diabetic rats. Data are mean percentage of baseline value $\pm \mathrm{SD}\left(n=9-10\right.$ per group). ${ }^{*} p<0.05$ versus control; $\uparrow p<0.05$ versus obese. $\mathrm{AF}=$ isometric active force normalized by cross-sectional area.

rats (11) (Fig. 4). The main mechanism involved is probably the down-regulation of $\beta_{1}$ - and $\beta_{2}$-adrenoceptors, which occurred without up-regulation of $\beta_{3}$-adrenoceptor and without modification in MRP4.

In Zucker obese rats, a single mutation $\mathrm{A}$ to $\mathrm{C}$ at nucleotide 880 (called "fa") in the leptin receptor gene on chromosome 5 causes a Gln to Pro substitution at residue 269 of the leptin receptor, leading to nonfunctional receptor. Zucker obese diabetic strain is a substrain selectively inbred for hyperglycemia, which carries an autosomal recessive defect in pancreatic $\beta$-cell transcription that is inherited independently of the fa mutation (25). The gene involved in this diabetic profile has not been yet identified but requires the fa mutation to induce diabetes. The Zucker obese rat model is associated with moderate hypertension without significant cardiac dysfunction, whereas the Zucker obese diabetic model is associated with no hypertension but mild diastolic cardiac dysfunction. We observed important metabolic changes with a different profile in obese and obese diabetic rats. These two strains are known to be associated with hyperlipidemia due to high low-density lipoprotein and high-density lipoprotein cholesterol, increased lipoprotein lipase activity, hyperinsulinemia, and insulin resistance, whereas hyperglycemia is observed only in the obese diabetic strain (male only) (25).

We did not observe noticeable myocardium dysfunction in obese and obese diabetic rats. AF was significantly increased in both obese and obese diabetic rats and associated with a prolongation of the contraction phase (increase in TPS and TPF). This prolongation of contraction has been previously reported in type I diabetic rats (15) and has been related to a slower cross-bridge cycling rate, a slower $\mathrm{Ca}^{2+}$ release from the $\mathrm{SR}$, and an alteration of $\mathrm{I}_{\text {to }}$ potassium current.

A slight decrease in the positive inotropic response of $\beta$-adrenergic stimulation was observed in obese rats and a marked decrease in obese diabetic rats (Fig. 1 and Table 2).
Conflicting results have been obtained in obese swine and obese rabbit (high-fat diet model) concerning cardiac $\beta$-adrenoceptor signaling pathway (9-11). Lima-Leopoldo et al (26) concluded that no change occurs in cardiac function after $\beta$-adrenoceptor stimulation in obese rats, but a significant decrease was observed only at the highest concentration of isoproterenol, and the magnitude of the effect was comparable to our results. Our results suggest that the impairment in $\beta$-adrenergic signaling pathway is less severe in obese than in obese diabetic rats and less severe in obese diabetic than that previously observed in type 1 diabetic rats (15).

The observed decreases in the responses to $\beta$-adrenoceptor stimulation in both obese and obese diabetic Zucker rats are consistently explained by the down-regulation of $\beta_{1}-$ and $\beta_{2}$-adrenoceptors (Fig. $3 A$ ). In Zucker obese rats, two earlier studies reported a decrease in $\beta$-adrenoceptor density but without separating $\beta$-adrenoceptor subtypes $(27,28)$. Our results markedly differ from those previously observed in type 1 diabetic rats (streptozotocin-induced diabetes) in which $\beta_{1}$-adrenoceptor down-regulation is associated with $\beta_{3}$-adrenoceptor up-regulation (15). This important difference may explain why the decrease in $\beta$-adrenoceptor stimulation was moderate in obese and obese diabetic Zucker rats in comparison to type 1 diabetic rats (15). It is well established now that the stimulation of $\beta_{3}$-adrenoceptors results in nitric oxide synthase-derived nitric oxide which is at the beginning of cyclic guanosine monophosphate production. Thereafter, cyclic guanosine monophosphate activates inhibitory G proteins and phosphodiesterases that increase the catabolism of the cAMP produced by the $\beta_{1}$ - and $\beta_{2}$-adrenoceptors stimulation (29) and thus results in a negative inotropic effect $(15,30)$. The down-regulation of $\beta_{1}$ - and $\beta_{2}$-adrenoceptors in the heart of obese rats is consistent with the chronic sympathetic activation associated with the metabolic syndrome (31) and the catecholamine resistance of adipose tissue (32). In contrast with type 1 diabetes (15), the lack of $\beta_{3}$-adrenoceptor overexpression observed in obese and obese diabetic rats did not impact the inotropic effect induced by $\beta_{1}$ - and $\beta_{2}$-adrenoceptors stimulation. In this way, we still observed a decreased response with dibutyryl cAMP administration in both obese and obese diabetic rats.

However, down-regulation of $\beta_{1}$-and $\beta_{2}$-adrenoceptors is probably not the sole mechanism involved in the decreased response to $\beta$-adrenoceptor stimulation. When the papillary muscles were directly stimulated by dibutyryl cAMP, we still observed a decreased response in both obese and obese diabetic rats, which were not significantly different between these two strains (Fig. 2). This suggests that some abnormality occurs downstream the adenylate cyclase level. Further studies are required to elucidate that mechanism, although our study clearly eliminates the role of MRP4 (Fig. 3E), which has been recently demonstrated to be overexpressed during aging and to contribute to the decrease in $\beta$-adrenoceptor stimulation observed in old rats (19). In the present study, the lack in overexpression of MRP4 and the modest decrease in cardiac contractile responsiveness observed in obese rats (Fig. 1) suggest 

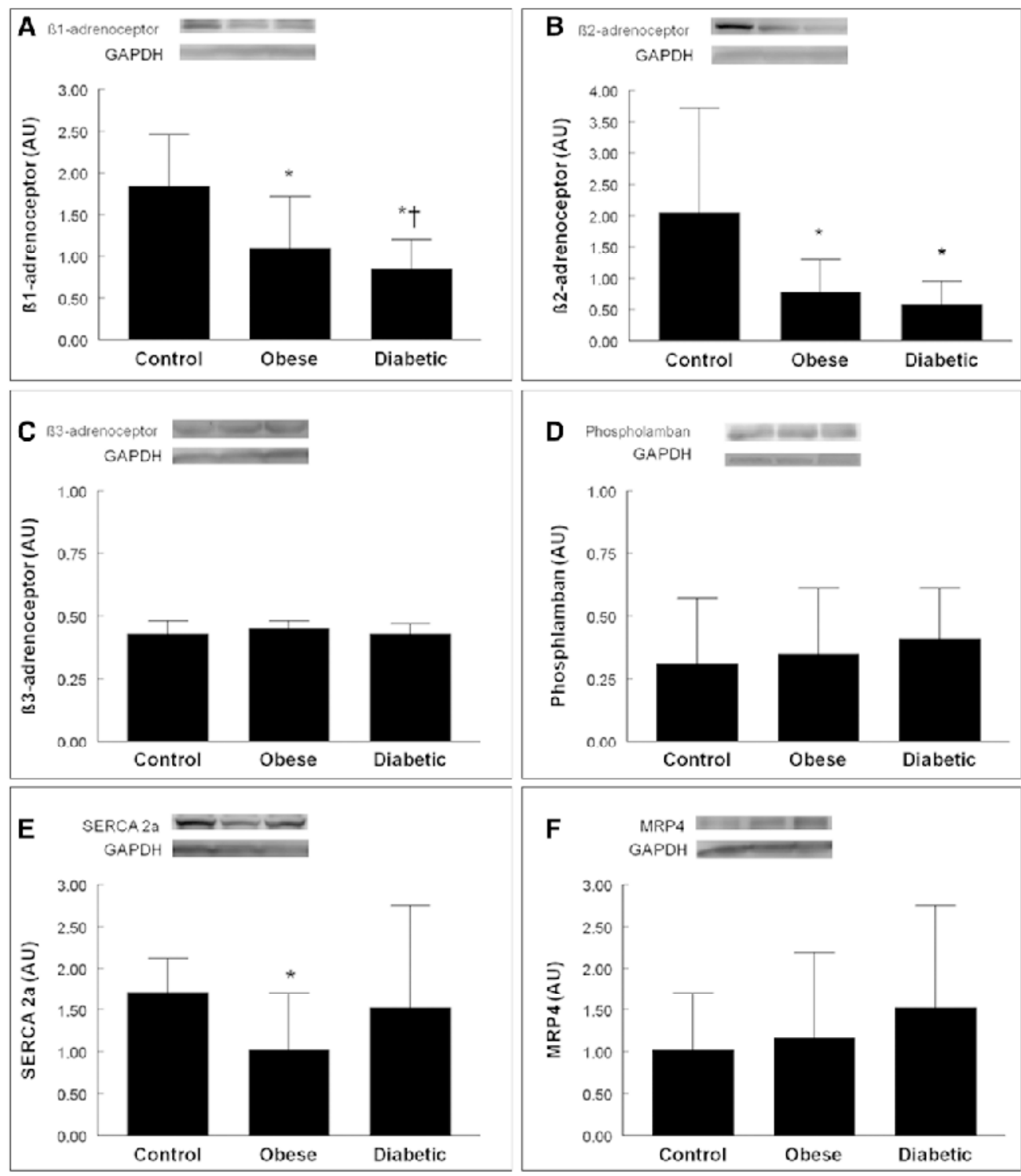

Figure 3. Representative Western blot and densitometric analysis reflecting $\beta_{1}$-adrenoceptor $(\mathbf{A}), \beta_{2}$ adrenoceptor (B), $\beta_{3}$-adrenoceptor (C), phospholamban (D), SERCA 2a (E), and multidrug resistanceassociated protein (MRP4) (F) protein expression in Zucker control, obese, and obese diabetic rats $(n=7$ in each group). Data are mean \pm SD. ${ }^{*} p<0.05$ versus control; $t p<0.05$ versus obese. GAPDH $=$ glyceraldehyde 3-phosphate dehydrogenase, SERCA2a = sarcoplasmic reticulum calcium ATPase.

was not modified in either obese or obese diabetic rats. The decrease in SERCA 2a, which occurred without significant changes in the SERCA 2a/phospholamban ratio, did not impact the positive lusitropic effect of $\beta$-adrenergic stimulation. These results are consistent with those reported in type 1 diabetic rats in which the lusitropic effects of $\beta$-adrenoceptor stimulation were preserved, despite diabetic cardiomyopathy (33). Such discrepancies between inotropic and lusitropic effects have been reported in other situations (24) and are probably related to the fact that smaller concentrations of cAMP are required to induce a maximal lusitropic effect (34).

Catecholamines are widely used in critically ill patients but considerable intra- and interindividual variability exists in the response. Most previous studies have tried to elucidate pharmacokinetic and pharmacodynamic differences in catecholamine response $(35,36)$, but very few have considered differences which may be linked to the baseline characteristics of the patient. Recently, Bauman et al (37) demonstrated that ethnic differences may be associated with significant difference in vasopressor requirements in patients with septic shock, but these authors did not look

that this mechanism is of limited importance. To support this conclusion, when the production of cAMP by adenylate cyclase was directly stimulated using forskolin (Fig. 2), we still observed a decreased response in both obese and obese diabetic rats, which was more pronounced in obese diabetic rats. These results contrast with those obtained in isolated membrane of heart cells in Zucker obese rats (25). This discrepancy may be explained by the use of isolated membrane preparation and not intact papillary muscle. Thus, it is likely that several other mechanisms participate in the impairment in the $\beta$-adrenergic signaling pathway, one situated at the level of adenylate cyclase and the other downstream, beside the down-regulation of $\beta_{1}$ - and $\beta_{2}$-adrenoceptors.

Lusitropy plays an important role in the maintenance of cardiac output. The lusitropic effect of $\beta$-adrenoceptor stimulation at genetic or phenotypic characteristics that might explain these differences. This study reported that the required doses of vasopressors were higher in Afro-American patients compared with white patients. However, the proportion of diabetic patients was also markedly different ( $68 \%$ vs $32 \%)$, and it is likely that the proportion of obese patients may also have been different, although this was not stated in the article. Future clinical research on the catecholamine requirements in the ICU should focus not only on genetic and phenotypic profile and catecholamine pharmacokinetics $(35,36)$ but also on the pathophysiologic conditions that may modify adrenoceptor signaling pathway.

The following limitations should be considered when assessing the clinical relevance of our results. First, this study was performed in rat myocardium, which differs from human myocardium. 


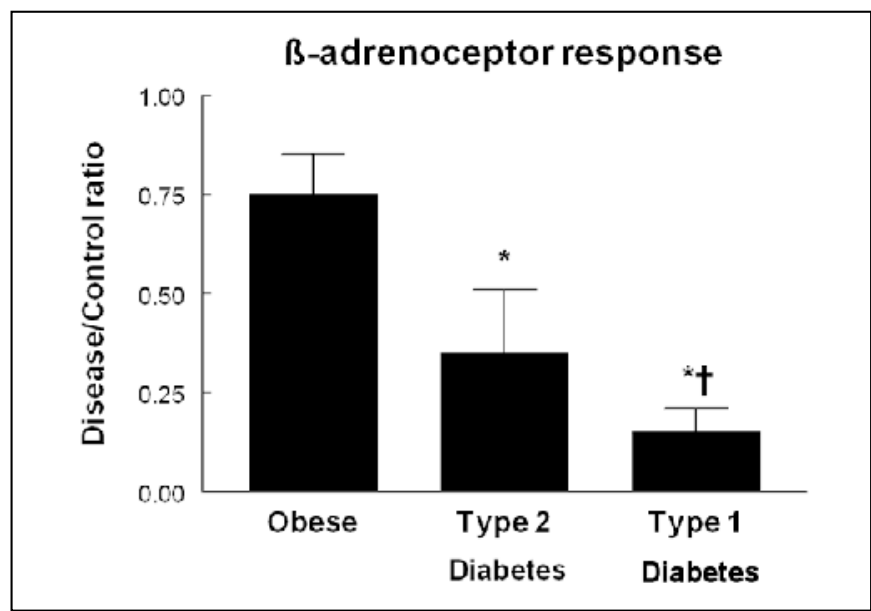

Figure 4. Comparison of the $\beta$-adrenergic stimulation (isoproterenol) response in Zucker obese, Zucker obese diabetic (type 2), and Wistar diabetic (type 1) rats. The ratio is the ratio of $\mathrm{Eff}_{\max }$ in the disease strain to $E$ in their respective control strain. Eff is the maximum effect on active isometric force of the concentration-effect curve of left ventricular papillary muscles exposed to increasing concentrations of isoproterenol. Data from type 1 diabetic rats (streptozotocin-induced diabetes) were obtained from Amour et al (15). Data are mean \pm SD. ${ }^{*} p<0.05$ versus Zucker obese; $\uparrow p<0.05$ versus Zucker obese diabetic rats.

Second, no animal model of obesity and diabetes completely mimicks diseases observed in humans, and thus, it is important to acknowledge the limitations of the leptin/leptin receptor-based rodent model. For example, Zucker obese rats have only moderate hypertension, do not develop premature atherosclerosis, and their endothelium-dependent relaxation is not impaired, and Zucker obese diabetic rats do not develop sympathetic neuroaxonal dystrophy (25). Thus, further studies in humans are required to validate our results. Third, we did not study changes observed after treatment of diabetes or food restriction in obesity. For example, it has been shown that food restriction may improve $\beta$-adrenergic stimulation in obese rats (28).

In conclusion, we observed a slight decrease in the positive inotropic effect of $\beta$-adrenoceptor stimulation in Zucker obese rats, which was more pronounced in Zucker obese diabetic rats and mainly related to the down-regulation of $\beta_{1}$ - and $\beta_{2}$-adrenoceptors.

\section{ACKNOWLEDGMENT}

We thank Dr. David Baker, DM, FRCA (Department of Anesthesiology and Critical Care, Hôpital Necker-Enfants Malades, Paris), for reviewing the article and Dr. Michèle Guerre-Millo (Research Director, UMR-S INSERM 1166, IHU ICAN, Sorbonne Universités UPMC Univ Paris 06, Paris, France) for scientific advice.

\section{REFERENCES}

1. Gallagher EJ, LeRoith D, Karnieli E: The metabolic syndrome-From insulin resistance to obesity and diabetes. Endocrinol Metab Clin North Am 2008; 37:559-579, vii

2. Glance LG, Wissler R, Mukamel DB, et al: Perioperative outcomes among patients with the modified metabolic syndrome who are undergoing noncardiac surgery. Anesthesiology 2010; 113:859-872

3. Pickkers P, de Keizer N, Dusseljee J, et al: Body mass index is associated with hospital mortality in critically ill patients: An observational cohort study. Crit Care Med 2013; 41:1878-1883
4. Prescott HC, Chang VW, O'Brien JM Jr, et al: Obesity and 1-year outcomes in older Americans with severe sepsis. Crit Care Med 2014; 42:1766-1774

5. Arabi YM, Dara SI, Tamim HM, et al; Cooperative Antimicrobial Therapy of Septic Shock (CATSS) Database Research Group: Clinical characteristics, sepsis interventions and outcomes in the obese patients with septic shock: An international multicenter cohort study. Crit Care 2013; 17:R72

6. Nelson J, Billeter AT, Seifert B, et al: Obese trauma patients are at increased risk of early hypovolemic shock: A retrospective cohort analysis of 1,084 severely injured patients. Crit Care 2012; 16:R77

7. Amour J, Kersten JR: Diabetic cardiomyopathy and anesthesia: Bench to bedside. Anesthesiology 2008; 108:524-530

8. Straznicky NE, Grima MT, Sari $\mathrm{Cl}$, et al: Neuroadrenergic dysfunction along the diabetes continuum: A comparative study in obese metabolic syndrome subjects. Diabetes 2012; 61:2506-2516

9. Dincer UD: Cardiac $\beta$-adrenoceptor expression is markedly depressed in Ossabaw swine model of cardiometabolic risk. Int J Gen Med 2011; 4:493-499

10. Carroll JF, Jones AE, Hester RL, et al: Reduced cardiac contractile responsiveness to isoproterenol in obese rabbits. Hypertension 1997; 30:1376-1381

11. Carroll JF, Kyser CK, Martin MM: $\beta$-adrenoceptor density and adenylyl cyclase activity in obese rabbit heart. Int J Obesity 2002; 26:627-632

12. Zucker LM, Zucker TF: Fatty a new mutation in the rat. J Heredity $1961 ; 52: 275-278$

13. Kanasaki K, Koya D: Biology of obesity: Lessons from animal models of obesity. J Biomed Biotechnol 2011; 2011:197636

14. Friedman JE, de Venté JE, Peterson RG, et al: Altered expression of muscle glucose transporter GLUT-4 in diabetic fatty Zucker rats (ZDF/Drt-fa). Am J Physiol 1991; 261:E782-E788

15. Amour J, Loyer X, Le Guen M, et al: Altered contractile response due to increased beta3-adrenoceptor stimulation in diabetic cardiomyopathy: The role of nitric oxide synthase 1-derived nitric oxide. Anesthesiology 2007; 107:452-460

16. Birenbaum A, Tesse A, Loyer X, et al: Involvement of beta 3-adrenoceptor in altered beta-adrenergic response in senescent heart: Role of nitric oxide synthase 1-derived nitric oxide. Anesthesiology 2008; 109:1045-1053

17. Sassi Y, Abi-Gerges A, Fauconnier J, et al: Regulation of cAMP homeostasis by the efflux protein MRP4 in cardiac myocytes. FASEB J 2012; 26:1009-1017

18. Carillion A, Feldman S, Jiang $C$, et al: Overexpression of cyclic adenosine monophosphate effluent protein MRP4 induces an altered response to $\beta$-adrenergic stimulation in the senescent rat heart. Anesthesiology 2015; 122:334-342

19. Allain CC, Poon LS, Chan CS, et al: Enzymatic determination of total serum cholesterol. Clin Chem 1974; 20:470-475

20. Fossati P, Prencipe L: Serum triglycerides determined colorimetrically with an enzyme that produces hydrogen peroxide. Clin Chem 1982; 28:2077-2080

21. Yee HY: Automated hexokinase procedure for assaying glucose in urine, serum, or plasma. Clin Chem 1972; 18:1416-1419

22. Riou $B$, Lecarpentier $Y$, Viars $P$ : Inotropic effect of ketamine on rat cardiac papillary muscle. Anesthesiology 1989; 71:116-125

23. Hanouz JL, Riou B, Massias L, et al: Interaction of halothane with alpha- and beta-adrenoceptor stimulations in rat myocardium. Anesthesiology 1997; 86:147-159

24. David JS, Vivien B, Lecarpentier Y, et al: Extracellular calcium modulates the effects of protamine on rat myocardium. Anesth Analg 2001; 92:817-823

25. Wang B, Chandrasekera PC, Pippin JJ: Leptin- and leptin receptordeficient rodent models: Relevance for human type 2 diabetes. Curr Diabetes Rev 2014; 10:131-145

26. Lima-Leopoldo AP, Leopoldo AS, Sugizaki MM, et al: Myocardial dysfunction and abnormalities in intracellular calcium handling in obese rats. Arq Bras Cardiol 2011; 97:232-240

27. Strassheim D, Houslay MD, Milligan G: Regulation of cardiac adenylate cyclase activity in rodent models of obesity. Biochem $J 1992$; 283(Part 1):203-208 
28. Chatelain $P$, Robberecht $P$, De Neef $P$, et al: Impairment of hormonestimulated cardiac adenylate cyclase activity in the genetically obese (fa/fa) Zucker rat. Pflugers Arch 1981; 390:10-16

29. Khamssi M, Brodde OE: The role of cardiac beta1- and beta2-adrenoceptor stimulation in heart failure. J Cardiovasc Pharmacol 1990; 16(Suppl 5):S133-S137

30. Gauthier C, Leblais V, Moniotte S, et al: The negative inotropic action of catecholamines: Role of beta3-adrenoceptors. Can J Physiol Pharmacol 2000; 78:681-690

31. Canale MP, Manca di Villahermosa S, Martino G, et al: Obesityrelated metabolic syndrome: Mechanisms of sympathetic overactivity. Int J Endocrinol 2013; 2013:865965

32. Reynisdottir $\mathrm{S}$, Wahrenberg $\mathrm{H}$, Carlström $\mathrm{K}$, et al: Catecholamine resistance in fat cells of women with upper-body obesity due to decreased expression of beta 2-adrenoceptors. Diabetologia 1994; 37:428-435
33. Amour J, Loyer X, Michelet $P$, et al: Preservation of the positive lusitropic effect of beta-adrenoceptors stimulation in diabetic cardiomyopathy. Anesth Analg 2008; 107:1130-1138

34. Coudray N, Beregi JP, Lecarpentier Y, et al: Effects of isoproterenol on myocardial relaxation rate: Influence of the level of load. $A m ~ J$ Physiol 1993; 265:H1645-H1653

35. Abboud I, Lerolle N, Urien S, et al: Pharmacokinetics of epinephrine in patients with septic shock: Modelization and interaction with endogenous neurohormonal status. Crit Care 2009; 13:R120

36. Beloeil H, Mazoit JX, Benhamou D, et al: Norepinephrine kinetics and dynamics in septic shock and trauma patients. $\mathrm{Br} J$ Anaesth 2005; 95:782-788

37. Bauman ZM, Killu KF, Rech MA, et al: Racial differences in vasopressor requirements for septic shock. Shock 2014; 41:188-192 


\section{Chapter Four}

\section{Limitations}

In our study, several limitations should be taken into consideration. Firstly, our study was conducted in rat myocardium. It differs from human myocardium. In rat myocardium, a negative staircase (increase in stimulation frequency decreases force) is observed, and the SR is more developed than in human. ${ }^{[273]}$ Thus, there would be some difficulties if applying our results to human myocardium. Secondly, no animal model of obesity or diabetes mellitus completely mimic diseases observed in human and thus it is important to acknowledge the limitations of the leptin/leptin receptor-based rodent models. Zucker obese rats have only moderate hypertension, do not develop premature atherosclerosis, and their endotheliumdependent relaxation is not impaired. Zucker diabetic rats do not develop sympathetic neuroaxonal dystrophy ${ }^{[295]}$. Thus further studies in humans are required to validate our results. Thirdly, we did not study changes observed after treatment of diabetes or food restriction in obesity. For example, it has been shown that food restriction may improve $\beta$-adrenergic stimulation in obese rats ${ }^{[205]}$. Fourthly, our study about rat myocardium in vitro was conducted at $29{ }^{\circ} \mathrm{C}$ because of the stability of the mechanical properties of the papillary muscles at this temperature. Meanwhile, this isolated left ventricular papillary muscle experiment can only observe the muscle performances without the inter-regulation of heart rates, loads, or wall geometry of heart in vivo ${ }^{[401]}$. Fifthly, echocardiography was conducted under isoflurane anesthesia, and previous studies reported that the halogenated anesthetic agents can interfere with $\beta$-adrenergic stimulation in healthy rats and many kinds of cardiomyopathy ${ }^{[253,275,402]}$. Although the potentiation of $\beta$-adrenoceptor stimulation was reported to be preserved with isoflurane in diabetic rats, our difference was still considered as the adjustment of neurohumoral compensatory mechanisms. 


\section{Chapter Five}

\section{Perspectives}

Although the extrapolation of our experimental research into clinical practice should be considered with caution, due to several limitations, these experiments shed a new light on the cardiac dysfunction of obesity with or without type 2 diabetes mellitus. Our experiment demonstrates one of the basic mechanisms related to the cardiac dysfunction in obesity and diabetes mellitus, that is the alteration in the $\beta$-adrenoceptor signaling pathway. There is a slight decrease in the positive inotropic effects under the $\beta$-adrenergic stimulation in the myocardium of obese rats, and the decrease is more significant in the myocardium of type 2 diabetic rats, which are related to the down - regulation of $\beta_{1}$ - and $\beta_{2}$-adrenoceptor proteins. Meanwhile, previous researches in our laboratory already showed that the down - regulation of $\beta_{1}$-adrenoceptor and up - regulation of $\beta_{3}$-adrenoceptor are associated with the decreased positive inotropic responses in type 1 diabetes mellitus ${ }^{[55,56]}$, which is mediated by $\mathrm{NOS}_{1}$ derived NO.

A recent study has shown the years of life lost from diabetes and cardiovascular disease in the very obese people could be up to 8 years, which was 6 years in the obese people and 3 years in the overweight ones ${ }^{[403]}$. In addition, the worst prognosis was in those who gained weight at young ages. So, it is urgent to further study the relative treatments to prevent the obesity and metabolic syndrome. In our experiment, we have not studied the influences on the cardiovascular functions after treatment to obesity or diabetes. It is worthwhile to continue the researches of the cardiac $\beta$-adrenergic signalling pathway under appropriate treatments.

Since the sympathetic nervous hyperactivity is one of the basic driving forces in human obesity and obesity - related disease, treatments to inhibit hyperactivity of sympathetic nervous system would be a useful method, including diet adjustments, physical exercises and some pharmacological treatments. The dietary weight loss and physical exercises are still the first line of therapy ${ }^{[2]}$. It has been shown that food restriction may improve $\beta$-adrenergic stimulation in obese rats ${ }^{[205]}$. The proportion of dietary components for the optimal cardiac health is still under research. The unsaturated fat is conventionally regarded as a "healthier" fat than saturated $\mathrm{fat}^{[46]}$. However, in the human with metabolic syndrome, researchers recently found that high intake of saturated fat in the context of a low carbohydrate intake still did not generate the accumulation of plasma saturated fat acid, which is a predictor of the 
increasing risk of diabetes and heart diseases ${ }^{[404]}$. Secondly, physical exercises, regardless of low or high training volumes, have shown no effects on the decreased $\beta$-adrenoceptor responsiveness in the obese animals ${ }^{[405,406]}$, even though the improvements in body fat, circulatory norepinephrine and hemodynamic status could be observed. In one experiment on the STZ - induced diabetic rats, researcher found that the exercise trainings initiated after the onset of diabetes could delay the deterioration of myocardial contractility, reduce the loss of $\beta_{1}$-adrenoceptor, and improve the responsiveness to the $\beta$-adrenergic stimulations in vivo ${ }^{[251]}$. Thirdly, some drugs with neuroadrenergic effects showed different results in the metabolic syndrome and heart diseases. For an instance, antihypertensive drug diuretics might worsen the insulin resistance status and exacerbate the sympathetic hyperactivity in metabolic syndrome ${ }^{[33,407]}$. The angiotensin - converting enzyme inhibitor (ACEI) or angiotensin receptor blockade (ARB) can improve insulin sensitivity and cause sympathoinhibitory effects $^{[407]}$. Statins and some hypoglycemic drugs were also found to exert sympathoinhibitory properities $^{[408]}$. The $\beta$-blockers, including carvedilol, metoprolol, bisopolol and nebivolol, showed the beneficial influences on the survival and left ventricular remodeling in the chronic heart failure ${ }^{[140,141]}$. Specific agonist or antagonist to $\beta_{3}$-adrenoceptor might be one strategy for different stage of heart failure ${ }^{[124]}$. As in our previous study, $\beta_{3}$-adrenoceptor inhibitor could partially restore the decreased positive inotropic effects under the $\beta$-adrenergic stimulations in diabetic rats in vitro ${ }^{[55]}$.

Catecholamines are widely used in critically ill patients but considerable intra- and interindividual variability exists in the response. Most previous studies tried to elucidate pharmacodynamics and pharmacodynamics difference in catecholamines response ${ }^{[409,410]}$, but very few considered differences linked to baseline characteristics of the patient. Recently, Bauman et al. ${ }^{[411]}$ demonstrated that ethnic differences may be associated with significant difference in vasopressor requirements in patients with septic shock, but overlooked the genetic or phenotypic characteristics that may explain these differences. This study reported that the required doses of vasopressors were higher in Afro-American patients compared with white patients. However, the proportion of diabetic patients was also markedly different (68 vs $32 \%$ ), and it is likely that the proportion of obese patients may also have been different, although this was not illustrated in the article. Future clinical research in ICU should focus not only on the catecholamine requirements according to both genetic and phenotypic profile, but also on the pathophysiological conditions that may both modify adrenoceptor signaling pathway and catecholamine pharmacokinetics ${ }^{[409,410]}$. 


\section{Chapter Six}

\section{Conclusions}

In the present study, we mainly confirmed the following conclusions:

1. According to the baseline values of transthoracic echocardiography (in vivo) and isolated left ventricular papillary muscles (in vitro), no alteration was observed in the baseline cardiac function of Zucker obese and obese diabetic rats.

2. The positive inotropic effects in response to $\beta$-adrenergic stimulations demonstrated a slight impairment in Zucker obese rats. This decrease in the positive inotropic effects to $\beta$ adrenergic stimulations was more pronounced in Zucker obese diabetic (type 2) rats. Comparing with the previous study of our laboratory, Zucker obese diabetic (type 2) rats showed less severe change in the positive inotropic effects than that previously observed in type 1 diabetic rats.

3. Those modifications were mainly associated with the down-regulation of $\beta_{1^{-}}$and $\beta_{2^{-}}$ adrenoceptor proteins, and without up-regulation of $\beta_{3}$-adrenoceptor protein.

4. Subtle impairments also occur upstream and downstream of the adenylate cyclase level of the $\beta$-adrenergic stimulation pathway in Zucker obese and obese diabetic rats, besides the down-regulation of $\beta_{1}$ - and $\beta_{2}$-adrenoceptor.

5. Those modifications were not due to the over-expression of MRP4. 


\section{Chapter Seven}

\section{References}

[1] Byrne CD, Wild SH. The metabolic syndrome // Byrne CD, Wild SH. The global burden of the metabolic syndrome and its consequences for diabetes and cardiovascular disease. Chichester: John Wiley \& Sons Ltd, 2005, 1-18.

[2] Lambert GW, Straznicky NE, Lambert EA, et al. Sympathetic nervous activation in obesity and the metabolic syndrome - Causes, consequences and therapeutic implications. Pharmacol Ther 2010; 126(2): 159-172.

[3] Grundy SM. Pre-diabetes, metabolic syndrome, and cardiovascular risk. J Am Coll Cardiol 2012; 59(7):635-643.

[4] Abdul-Ghani MA, Williams K, DeFronzo RA, et al. What is the best predictor of future type 2 diabetes? Diabetes Care 2007; 30(6): 1544-1548.

[5] Ford ES, Giles WH, Dietz WH. Prevalence of the metabolic syndrome among US adults: findings from the third National Health and Nutrition Examination Survey. JAMA 2002; 287(3): 356-359.

[6] Cameron AJ, Shaw JE, Zimmet PZ. The metabolic syndrome: prevalence in worldwide populations. Endocrinol Metab Clin North Am 2004; 33(2): 351-375.

[7] Campos DH, Leopoldo AS, Lima-Leopoldo AP, et al. Obesity preserves myocardial function during blockade of the glycolytic pathway. Arq Bras Cardiol 2014; 103(4): 330-337.

[8] Danaei G, Finucane MM, Lu Y, et al. National, regional, and global trends in fasting plasma glucose and diabetes prevalence since 1980: systematic analysis of health examination surveys and epidemiological studies with 370 country-years and 2.7 million participants. Lancet 2011; 378(9785): 31-40.

[9] Levy D, Garrison RJ, Savage DD, et al. Prognostic implications of echocardiographically determined left ventricular mass in the Framingham Heart Study. N Engl J Med 1990; 322(22): 1561-1566.

[10] Leopoldo AS, Lima-Leopoldo AP, Sugizaki MM, et al. Involvement of L-type calcium channel and SERCA2a in myocardial dysfunction induced by obesity. J Cell Physiol 2011; 226(11): 2934-2942.

[11] Poirier P, Giles TD, Bray GA, et al. Obesity and cardiovascular disease: Pathophysiology, evaluation, and effect of weight loss: an update of the 1997 American Heart Association 
Scientific Statement on obesity and heart disease from the Obesity Committee of the Council on Nutrition, Physical Activity, and Metabolism. Circulation 2006; 113(6): 898-918.

[12] Kenchaiah S, Evans JC, Levy D, et al. Obesity and the risk of heart failure. N Engl J Med 2002; 347(5): 305-313.

[13] Horwich TB, Fonarow GC, Hamilton MA, et al. The relationship between obesity and mortality in patients with heart failure. J Am Coll Cardiol 2001; 38(3): 789-795.

[14] Lavie CJ, Osman AF, Milani RV, et al. Body composition and prognosis in chronic systolic heart failure: the obesity paradox. Am J Cardiol 2003; 91(7): 891-894.

[15] Iacobellis G, Sharma AM. Obesity and the heart: redefinition of the relationship. Obes Rev 2007; 8(1): 35-39.

[16] Daniels A, van Bilsen M, Janssen BJ. Impaired cardiac functional reserve in type 2 diabetic $\mathrm{db} / \mathrm{db}$ mice is associated with metabolic, but not structural, remodeling. Acta Physiol (Oxf) 2010; 200(1):11-22.

[17] Rubler S, Dlugash J, Yuceoglu YZ, et al. New type of cardiomyopathy associated with diabetic glomerulosclerosis. Am J Cardiol 1972; 30(6): 595-602.

[18] Schannwell CM, Schneppenheim M, Perings S, et al. Left ventricular diastolic dysfunction as an early manifestation of diabetic cardiomyopathy. Cardiology 2002; 98(12):33-39.

[19] He J, Ogden LG, Bazzano LA, et al. Risk factors for congestive heart failure in US men and women: NHANES I epidemiologic follow-up study. Arch Intern Med 2001; 161(7): 9961002 .

[20] Lakka HM, Laaksonen DE, Lakka TA, et al. The metabolic syndrome and total and cardiovascular disease mortality in middle-aged men. JAMA 2002; 288(21):2709-2716.

[21] Sattar N, Gaw A, Scherbakova O, et al. Metabolic syndrome with and without C-reactive protein as a predictor of coronary heart disease and diabetes in the West of Scotland Coronary Prevention Study. Circulation 2003; 108(4):414-419.

[22] Malik S, Wong ND, Franklin SS, et al. Impact of the metabolic syndrome on mortality from coronary heart disease, cardiovascular disease, and all causes in United States adults. Circulation 2004; 110(10):1245-1250.

[23] Aschner P. Metabolic syndrome as a risk factor for diabetes. Expert Rev Cardiovasc Ther 2010; 8(3):407-412.

[24] National Cholesterol Education Program (NCEP) Expert Panel on Detection, Evaluation, and Treatment of High Blood Cholesterol in Adults (Adult Treatment Panel III). Third Report of the National Cholesterol Education Program (NCEP) Expert Panel on Detection, 
Evaluation, and treatment of High Blood Cholesterol in Adults (Adult Treatment Panel III) final report. Circulation 2002; 106(25): 3143-3421.

[25] Heidemann C, Boeing H, Pischon T, et al. Association of a diabetes risk score with risk of myocardial infarction, stroke, specific types of cancer, and mortality: a prospective study in the European Prospective Investigation into Cancer and Nutrition (EPIC)-Potsdam cohort. Eur J Epidemiol 2009; 24(6): 281-288.

[26] Preis SR, Hwang SJ, Coady S, et al. Trends in all-cause and cardiovascular disease mortality among women and men with and without diabetes mellitus in the Framingham heart study, 1950 to 2005. Circulation 2009; 119(13): 1728-1735.

[27] Huggett RJ, Scott EM, Gilbey SG, et al. Impact of type 2 diabetes mellitus on sympathetic neural mechanisms in hypertension. Circulation 2003; 108(25): 3097-3101.

[28] Grassi G, Quarti-Trevano F, Seravalle G, et al. Cardiovascular risk and adrenergic overdrive in the metabolic syndrome. Nutr Metab Cardiovasc Dis 2007; 17(6): 473-481.

[29] Esler M, Straznicky N, Eikelis N, et al. Mechanisms of sympathetic activation in obesity - related hypertension. Hypertension 2006; 48(5): 787-796.

[30] Grassi G, Cattaneo BM, Seravalle G, et al. Baroreflex control of sympathetic nerve activity in essential and secondary hypertension. Hypertension 1998; 31(1):68-72.

[31] Grassi G, Seravalle G, Cattaneo BM, et al. Sympathetic activation in obese normotensive subjects. Hypertension 1995; 25(4 Pt 1):560-563.

[32] Canale MP, Manca di Villahermosa S, Martino G, et al. Obesity-related metabolic syndrome: mechanisms of sympathetic overactivity. Int J Endocrinol 2013; 2013: 865965.

[33] Mancia G, Bousquet P, Elghozi JL, et al. The sympathetic nervous system and the metabolic syndrome. J Hypertens 2007; 25(5): 909-920.

[34] Reaven GM. Role of insulin resistance in human disease. Diabetes 1988; 37(12): 15951607.

[35] Reaven GM. Pathophysiology of insulin resistance in human disease. Physiol Rev 1995; 75(3): 473-486.

[36] Smith MM, Minson CT. Obesity and adipokines: effects on sympathetic overactivity. J Physiol 2012; 590(Pt8): 1787-1801.

[37] Kalil GZ, Haynes WG. Sympathetic nervous system in obesity-related hypertension: mechanisms and clinical implications. Hypertens Res 2012; 35(1): 4-16.

[38] Masuo K. Roles of Beta2- and Beta3-Adrenoceptor Polymorphisms in Hypertension and Metabolic Syndrome. Int J Hypertens 2010; 2010: 832821. 
[39] Masuo K. Adrenoceptor Polymorphisms in Hypertension and Diabetes with obesityupdate in 2014. Curr Hypertens Rev 2014. [Epub ahead of print]

[40] Hall JE, da Silva AA, do Carmo JM, et al. Obesity-induced hypertension: role of sympathetic nervous system, leptin, and melanocortins. J Biol Chem 2010; 285(23): 1727117276.

[41] Vaz M, Jennings G, Turner A, et al. Regional sympathetic nervous activity and oxygen consumption in obese normotensive human subjects. Circulation 1997; 96(10): 3423-3429.

[42] De Lucia C, Femminella GD, Gambino G, et al. Adrenal adrenoceptors in heart failure. Front Physiol 2014; 5: 246.

[43] Carroll JF, Jones AE, Hester RL, et al. Reduced cardiac contractile responsiveness to isoproterenol in obese rabbits. Hypertension 1997; 30(6): 1376-1381.

[44] Lima-Leopoldo AP, Leopoldo AS, Sugizaki MM, et al. Myocardial dysfunction and abnormalities in intracellular calcium handling in obese rats. Arq Bras Cardiol 2011; 97(3): $232-240$.

[45] Carroll JF, Zenebe WJ, Strange TB. Cardiovascular function in a rat model of dietinduced obesity. Hypertension 2006; 48(1):65-72.

[46] Okere IC, Chandler MP, Mcelfresh TA, et al. Differential effects of saturated and unsaturated fatty acid diets on cardiomyocyte apoptosis, adipose distribution, and serum leptin. Am J Physiol Heart Circ Physiol 2006; 291(1): H38-H44.

[47] Ricci E, Smallwood S, Chouabe C, et al. Electrophysiological characterization of left ventricular myocytes from obese Sprague-Dawley rat. Obesity 2006; 14(5): 778-786.

[48] Ouwens DM, Boer C, Fodor M, et al. Cardiac dysfunction induced by high-fat diet is associated with altered myocardial insulin signaling in rats. Diabetologia 2005; 48(6): 12291237.

[49] Carroll JF, Summers RL, Dzielak DJ, et al. Diastolic compliance is reduced in obese rabbits. Hypertension 1999; 33(3): 811-815.

[50] Relling DP, Esberg LB, Fang CX, et al. High-fat-diet-induced juvenile obesity leads to cardiomyocyte dysfunction and upregulation of Foxo3a transcription factor independent of lipotoxicity and apoptosis. J Hypertens 2006; 24(3): 549-561.

[51] Lima-Leopoldo AP, Sugizaki MM, Leopoldo AS, et al. Obesity induces upregulation of genes involved in myocardial Ca2+ handling. Braz J Med Biol Res 2008; 41(7): 615-620.

[52] Ren J, Walsh MF, Jefferson L, et al. Basal and ethanol-induced cardiac contractile response in lean and obese Zucker rat hearts. J Biomed Sci 2000; 7(5): 390-400. 
[53] Young ME, Guthrie PH, Razeghi P, et al. Impaired long-chain fatty acid oxidation and contractile dysfunction in the obese Zucker rat heart. Diabetes 2002; 51(8): 2587-2595.

[54] Strassheim D, Houslay MD, Milligan G. Regulation of cardiac adenylate cyclase activity in rodents models of obesity. Biochem J 1992; 283(Pt 1): 203-208.

[55] Amour J, Loyer X, Le Guen M, et al. Altered contractile response due to increased beta3adrenoceptor stimulation in diabetic cardiomyopathy: the role of nitric oxide synthase 1derived nitric oxide. Anesthesiology 2007; 107(3): 452-460.

[56] Amour J, Loyer X, Michelet P, et al. Preservation of the positive lusitropic effect of betaadrenoceptors stimulation in diabetic cardiomyopathy. Anesth Analg 2008; 107(4): 11301138.

[57] Bratkovsky SV, Aasum E, Riemersma RA, et al. Reduced coronary reserve in response to short-term ischaemia and vasoactive drugs in ex vivo hearts from diabetic mice. Acta Physiol (Oxf) 2006; 186(3): 171-177.

[58] Carley AN, Semeniuk LM, Shimoni Y, et al. Treatment of type 2 diabetic db/db mice with a novel PPARgamma agonist improves cardiac metabolism but not contractile function. Am J Physiol Endocrinol Metab 2004; 286(3):E449-E455.

[59] Reyes M, Steinhelper ME, Alvarez JA, et al. Impact of physiological variables and genetic background on myocardial frequency-resistivity relations in the intact beating murine heart. Am J Physiol Heart Circ Physiol 2006; 291(4): H1659-H1669.

[60] Yue P, Arai T, Terashima M, et al. Magnetic resonance imaging of progressive cardiomyopathic changes in the db/db mouse. Am J Physiol Heart Circ Physiol 2007; 292(5): H2106-H2118.

[61] Fang ZY, Prins JB, Marwick TH. Diabetic cardiomyopathy: evidence, mechanisms, and therapeutic implications. Endocr Rev 2004; 25(4): 543-567.

[62] Kamata K, Satoh T, Matsumoto T, et al. Enhancement of methoxamine-induced contractile responses of rat ventricular muscle in streptozotocin-induced diabetes is associated with alpha1A adrenoceptor upregulation. Acta Physiol (Oxf) 2006; 188(3-4): 173-183.

[63] Boudina S, Abel ED. Diabetic cardiomyopathy revisited. Circulation 2007; 115(25): $3213-3223$.

[64] Aneja A, Tang WH, Bansilal S, et al. Diabetic cardiomyopathy: insights into pathogenesis, diagnostic challenges, and therapeutic options. Am J Med 2008; 121(9): 748757. 
[65] Op Den Buijs J, Ligeti L, Ivanics T, et al. Mathematical modeling of the calcium-left ventricular pressure relationship in the intact diabetic rat heart. Acta Physiol (Oxf) 2008; 193(3): 205-217.

[66] Essop MF, Anna Chan WY, Valle A, et al. Impaired contractile function and mitochondrial respiratory capacity in response to oxygen deprivation in a rat model of prediabetes. Acta Physiol(Oxf) 2009; 197(4): 289-296.

[67] Glance LG, Wissler R, Mukamel DB, et al. Perioperative outcomes among patients with the modified metabolic syndrome who are undergoing noncardiac surgery. Anesthesiology 2010; 113(4): 859-872.

[68] Pickkers P, de Keizer N, Dusseljee J, et al. Body mass index is associated with hospital mortality in critically ill patients: An observational cohort study. Crit Care Med 2013; 41(8): $1878-1883$.

[69] Prescott HC, Chang VW, O'Brien JM Jr, et al. Obesity and 1-year outcomes in older Americans with severe sepsis. Crit Care Med 2014; 42(8): 1766-1774.

[70] Arabi YM, Dara SI, Tamim HM, et al. Cooperative Antimicrobial Therapy of Septic Shock (CATSS) Database Research Group: Clinical characteristics, sepsis interventions and outcomes in the obese patients with septic shock: An international multicenter cohort study. Crit Care 2013; 17(2): R72.

[71] Nelson J, Billeter AT, Seifert B, et al. Obese trauma patients are at increased risk of early hypovolemic shock: A retrospective cohort analysis of 1,084 severely injured patients. Crit Care 2012; 16(3): R77.

[72] Straznicky NE, Grima MT, Sari CI, et al. Neuroadrenergic dysfunction along the diabetes continuum: a comparative study in obese metabolic syndrome subjects. Diabetes 2012; 61(10): 2506-2516.

[73] Birenbaum A, Tesse A, Loyer X, et al. Involvement of beta 3-adrenoceptor in altered beta-adrenergic response in senescent heart: role of nitric oxide synthase 1-derived nitric oxide. Anesthesiology 2008; 109(6): 1045-1053.

[74] Sassi Y, Abi-Gerges A, Fauconnier J, et al. Regulation of cAMP homeostasis by the efflux protein MRP4 in cardiac myocytes. FASEB J 2012; 26(3): 1009-1017.

[75] Carillion A, Feldman S, Jiang C, et al.Over-expression of cyclic adenosine monophosphate effluent protein MRP4 induces an altered response to $\beta$-adrenergic stimulation in the senescent rat heart. Anesthesiology 2015; 122(2): 334-342.

[76] Zucker LM, Zucker TF. Fatty, a new mutation in the rat. J Hered 1961; 52(6): 275-278. 
[77] Freedman JE, De Vente JE, Peterson RG, et al. Altered expression of muscle glucose transporter GLUT-4 in diabetic fatty Zucker rats (ZDF/Drt-fa). Am J Physiol 1991; 261(6 Pt 1): E782-E788.

[78] Davis LM, Michaelides M, Cheskin LJ, et al. Bromocriptine administration reduces hyperphagia and adiposity and differentially affects dopamine D2 receptor and transporter binding in leptin-receptor-deficient Zucker rats and rats with diet-induced obesity. Neuroendocrinology 2009; 89(2): 152-162.

[79] Zucker LM, Antoniades HN. Insulin and obesity in the Zucker genetically obese rat “fatty". Endocrinology 1972; 90(5): 1320-1330.

[80] Schreihofer AM, Mandel DA, Mobley SC, et al. Impairment of sympathetic baroreceptor reflexes in obese Zucker rats. Am J Physiol Heart Circ Physio 2007; 293(4): H2543-H2549.

[81] Marsh SA, Powell PC, Agarwal A, et al. Cardiovascular dysfunction in Zucker obese and Zucker diabetic fatty rats: role of hydronephrosis. Am J Physiol Heart Circ Physiol 2007; 293(1): H292-H298.

[82] Opie LH. Heart physiology: from cell to circulation. 4th ed. Philadelphia: Lippincott Williams \& Wilkins. 2004.

[83] Ahlquist RP. A study of the adrenotropic receptors. Am J Physiol 1948; 153(3): 586-600.

[84] Rockman HA, Koch WJ, Lefkowitz RJ. Seven-transmembrane-spanning receptors and heart function. Nature 2002; 415(6868): 206-212.

[85] Brodde OE, Bruck H, Leineweber K. Cardiac adrenoceptors: physiological and pathophysiological relevance. J Pharmacol Sci 2006; 100(5): 323-337.

[86] Minatoguchi S, Ito H, Ishimura K, et al. Modulation of noradrenaline release through presynaptic $\alpha_{2}$-adrenoceptors in congestive heart failure. Am Heart J 1995; 130(3 Pt 1): 516 521.

[87] Lands AM, Arnold A, McAuliff JP, et al. Differentiation of receptor systems activated by sympathomimetic amines. Nature 1967; 214(5088): 597-598.

[88] Del Monte F, Kaumann AJ, Poole-Wilson PA, et al. Coexistence of functioning beta 1and beta 2-adrenoceptors in single myocytes from human ventricle. Circulation 1993; 88(3): 854-863.

[89] Brodde OE, Michel MC. Adrenergic and muscarinic receptors in the human heart. Pharmacol Rev 1999; 51(4):651-689.

[90] Communal C, Singh K, Sawyer DB, et al. Opposing effects of beta(1)- and beta(2)adrenergic receptors on cardiac myocyte apoptosis: role of a pertussis toxin-sensitive Gprotein. Circulation 1999; 100(22): 2210-2212. 
[91] Zaugg M, Xu W, Lucchinetti E, et al. $\beta$-Adrenergic receptor subtypes differentially affect apoptosis in adult rat ventricular myocytes. Circulation 2000; 102(3): 344-350.

[92] Zhu WZ, Zheng M, Koch WJ, et al. Dual modulation of cell survival and cell death by $\beta_{2}$-adrenergic signaling in adult mouse cardiac myocytes. Proc Natl Acad Sci U S A 2001; 98(4): 1607-1612.

[93] Shizukuda Y, Buttrick PM. Subtype specific roles of $\beta$-adrenergic receptors in apoptosis of adult rat ventricularmyocytes. J Mol Cell Cardiol 2002; 34(7): 823-831.

[94] Pönicke K, Heinroth-Hoffmann I, Brodde OE. Role of $\beta_{1^{-}}$and $\beta_{2}$-adrenoceptors in hypertrophic and apoptotic effects of noradrenaline and adrenaline in adult rat ventricular cardiomyocytes. Naunyn Schmiedebergs Arch Pharmacol 2003; 367(6): 592-599.

[95] Trafford AW, Diaz ME, Eisner DA. Coordinated control of cell $\mathrm{Ca}(2+)$ loading and triggered release from the sarcoplasmic reticulum underlies the rapid inotropic response to increased L-type $\mathrm{Ca}(2+)$ current. Circ Res 2001; 88(2): 195-201.

[96] Carroll JF. Post-beta-receptor defect in isolated hearts of obese-hypertensive rabbits. Int J Obes Relat Metab Disord 1999; 23(8): 863-868.

[97] Bers DM. Cardac excitation-contraction coupling. Nature 2002; 415(6868): 198-205.

[98] Frank KF, Bölck B, Erdmann E, et al. Sarcoplasmic reticulum $\mathrm{Ca}^{2+}$-ATPase modulates cardiac contraction and relaxation. Cardiovasc Res 2003; 57(1): 20-27.

[99] Periasamy M, Bhupathy P, Babu GJ. Regulation of sarcoplasmic reticulum $\mathrm{Ca}^{2+}$ ATPase pump expression and its relevance to cardiac muscle physiology and pathology. Cardiovasc Res 2008; 77(2): 265-273.

[100] Zheng M, Han QD, Xiao RP. Distinct $\beta$-adrenergic receptor subtype signaling in the heart and their pathophysiological relevance. Acta Physiologica Sinica 2004; 56(1) : 1-15.

[101] Xiao RP, Avdonin P, Zhou YY, et al. Coupling of $\beta_{2}$-adrenoceptor to Gi proteins and its physiological relevance in murine cardiac myocytes. Circ Res 1999; 84(1): 43-52.

[102] Bond RA, Leff P, Johnson TD, et al. Physiological effects of inverse agonists in transgenic mice with myocardial overexpression of the $\beta_{2}$-adrenoceptor. Nature 1995; 374(6519): 272-276.

[103] Rohrer DK, Desai KH, Jasper JR, et al. Targeted disruption of the mouse b1-adrenergic receptor gene: developmental and cardiovascular effects. Proc Natl Acad Sci U S A 1996; 93(14): 7375-7380.

[104] Milano CA, Allen LF, Rockman HA, et al. Enhanced myocardial function in transgenic mice overexpressing the beta 2-adrenergic receptor. Science 1994; 264(5158): 582-586. 
[105] Du XJ, Vinean E, Woodcock DM, et al. Response to cardiac sympathetic activation in transgenic mice overexpressing beta 2-adrenergic receptor. Am J Physiol 1996; 271(2 Pt 2): H630-H636.

[106] Lemoine H, Ehle B, Kaumann AJ. Direct labelling of b2-adrenoceptor: comparison of binding potency of $3 \mathrm{H}-\mathrm{ICI} 118,551$ and blocking potency of ICI 118,551. Naunyn Schmiedebergs Arch Pharmacol 1985; 331(1): 40-51.

[107] Xiao RP, Ji X, Lakatta EG. Functional coupling of the $\beta_{2}$-adrenoceptor to a pertussis toxin-sensitive G protein in cardiac myocytes. Mol Pharmacol 1995; 47(2): 322-329.

[108] Xiao RP , Cheng H, Zhou YY, et al. Recent advances in cardiac beta2-adrenergic signal transduction. Circ Res 1999; 85(11): 1092-1100.

[109] Steinberg SF. The molecular basis for distinct $\beta$-adrenergic receptor subtype action in cardiomyocytes. Circ Res 1999; 85(11): 1101-1111.

[110] Kilts JD, Gerhardt MA, Richardson MD, et al. $\beta_{2}$-Adrenergic and several other G protein-coupled receptors in human atrial membranes activate both Gs and Gi. Circ Res 2000; 87(8): 705-709.

[111] Kaumann AJ, Sanders L, Lynham JA, et al. $\beta_{2}$-Adrenoceptor activation by zinterol causes protein phosphorylation, contractile effects and relaxant effects through a cAMP pathway in human atrium. Mol Cell Biochem 1996; 163-164: 113-123.

[112] Kaumann A, Bartel S, Molenaar P, et al. Activation of $\beta_{2}$-adrenergic receptors hastens relaxation and mediates phosphorylation of phospholamban, troponin I, and C-protein in ventricular myocardium from patients with terminal heart failure. Circulation 1999; 99(1): 6572.

[113] Molenaar P, Bartel S, Cochrane A, et al. Both $\beta_{2}$ - and $\beta_{1}$-adrenergic receptors mediate hastened relaxation and phosphorylation of phospholamban and troponin $I$ in ventricular myocardium of Fallot infants, consistent with selective coupling of $\beta_{2}$-adrenergic receptors to Gs-protein. Circulation 2000; 102(15): 1814-1821.

[114] Xiao RP, Zhang SJ, Chakir K, et al. Enhanced Gi signaling selectively negates $\beta_{2^{-}}$ adrenergic receptor (AR)- but not $\beta_{1}$-AR-mediated positive inotropic effect in myocytes from failing rat hearts. Circulation 2003; 108(13): 1633-1639.

[115] Pönicke K, Schlüter K-D, Heinroth-Hoffmann I, et al. Noradrenaline-induced increase in protein synthesis in adult rat cardiomyocytes: involvement of $\alpha_{1 \mathrm{~A}}$-adrenoceptors. Naunyn Schmiedebergs Arch Pharmacol 2001; 364(5): 444-453. 
[116] Pönicke K, Gröner F, Heinroth-Hoffmann I, et al. Agonist-specific activation of the $\beta_{2}$ adrenoceptor/Gs-protein and the $\beta_{2}$-adrenoceptor/Gi-protein pathway in adult rat ventricular cardiomoyctyes. Br J Pharmacolol 2006; 147(7): 714-719.

[117] Flatt A, Crane J, Purdie G, et al. The cardiovascular effects of beta adrenergic agonist drugs administered by nebulisation. Postgrad Med J 1990; 66(772): 98-101.

[118] Gong H, Sun H, Koch WJ, et al. Specific $\beta_{2}$ AR blocker ICI 118,551 actively decreases contraction through a Gi-coupled form of the $\beta_{2} \mathrm{AR}$ in myocytes from failing human heart. Circulation 2002; 105(21): 2497-2503.

[119] Rozec B, Gauthier C. Beta3-adrenoceptors in the cardiovascular system: putative roles in human pathologies. Pharmacol Ther 2006; 111(3): 652-673.

[120] Pavoine C, Defer N. The cardiac beta2-adrenergic signaling a new role for the cPLA2. Cell Signal 2005; 17(2): 141-152.

[121] Newton GE, Azevedo ER, Parker JD. Inotropic and sympathetic responses to the intracoronary infusion of a beta2-receptor agonist: a human in vivo study. Circulation 1999; 99(18): 2402-2407.

[122] Tevaearai HT, Eckhart AD, Walton GB, et al. Myocardial gene transfer and overexpression of beta2-adrenergic receptors potentiates the functional recovery of unloaded failing hearts. Circulation 2002; 106(1): 124-129.

[123] Rouget C, Breuiller-Fouche M, Mercier FJ, et al. The human near-term myometrial beta 3- adrenoceptor but not the beta 2-adrenoceptor is resistant to desensitization after sustained agonist stimulation. Br J Pharmacol 2004; 141(5): 831-841.

[124] Gauthier C, Rozec B, Manoury B, et al. Beta-3 adrenoceptors as new therapeutic targets for cardiovascular pathologies. Curr Heart Fail Rep 2011; 8(3): 184-192.

[125] Rozec B, Erfanian M, Laurent K, et al. Nebivolol, a vasodilating selective beta(1)blocker, is a beta(3)-adrenoceptor agonist in the nonfailing transplanted human heart. J Am Coll Cardiol 2009; 53(17): 1532-1538.

[126] Gauthier C, Langin D, Balligand JL. Beta3-adrenoceptors in the cardiovascular system. Trends Pharmacol Sci 2000; 21(11): 426-431.

[127] Wheeldon NM, McDevitt DG, Lipworth BJ. Cardiac effects of the beta 3-adrenoceptor agonist BRL35135 in man. Br J Clin Pharmacol 1994; 37(4): 363-369.

[128] Audigane L, Kerfant BG, El Harchi A, et al. Rabbit, a relevant model for the study of cardiac beta 3-adrenoceptors. Exp Physiol 2009; 94(4): 400-411.

[129] Barbier J, Mouas C, Rannou-Bekono F, et al. Existence of beta (3)-adrenoceptors in rat heart: functional implications. Clin Exp Pharmacol Physiol 2007; 34(8): 796-798. 
[130] Angelone T, Filice E, Quintieri AM, et al. Beta3-adrenoceptors modulate left ventricular relaxation in the rat heart via the NO-cGMP-PKG pathway. Acta Physiol (Oxf) 2008; 193(3): 229-239.

[131] Germack R, Dickenson JM. Induction of \{beta\}3-adrenergic receptor functional expression following chronic stimulation with noradrenaline in neonatal rat cardiomyocytes. $\mathbf{J}$ Pharmacol Exp Ther 2006; 316(1): 392-402.

[132] Gauthier C, Tavernier G, Trochu JN. Interspecies differences in the cardiac negative inotropic effects of beta(3)-adrenoceptor agonists. J Pharmacol Exp Ther 1999; 290(2): 687693.

[133] Chaudhry A, MacKenzie RG, Georgic LM. Differential interaction of beta 1- and beta 3-adrenergic receptors with Gi in rat adipocytes. Cell Signal 1994; 6(4): 457-465.

[134] Begin-Heick N. Beta 3-adrenergic activation of adenylyl cyclase in mouse white adipocytes: modulation by GTP and effect of obesity. J Cell Biochem 1995; 58(4): 464-473.

[135] Gauthier C, Leblais V, Kobzik L. The negative inotropic effect of beta3-adrenoceptor stimulation is mediated by activation of a nitric oxide synthase pathway in human ventricle. $\mathrm{J}$ Clin Invest 1998; 102(7): 1377-1384.

[136] Moniotte S, Kobzik L, Feron O, et al. Upregulation of beta(3)-adrenoceptors and altered contractile response to inotropic amines in human failing myocardium. Circulation 2001; 103(12): 1649-1655.

[137] Kaumann AJ, Molenaar P. Modulation of human cardiac function through $4 \beta$ adrenoceptor populations. Naunyn Schmiedebergs Arch Pharmacol 1997; 355(6): 667-681.

[138] Brodde OE, Bruck H, Leineweber K, et al. Presence, distribution and physiological function of adrenergic and muscarinic receptor subtypes in the human heart. Basic Res Cardiol 2001; 96(6): 528-538.

[139] Molenaar P, Parsonage WA. Fundamental considerations of $\beta$-adrenoceptor subtypes in human heart failure. Trends Pharmacol Sci 2005; 26(7): 368-375.

[140] Effect of metoprolol CR/XL in chronic heart failure: Metoprolol CR/XL Randomised Intervention Trial in Congestive Heart Failure (MERIT-HF). Lancet 1999; 353(9169): 20012007.

[141] Bristow MR, Gilbert EM, Abraham WT, et al. Carvedilol produces dose-related improvements in left ventricular function and survival in subjects with chronic heart failure. MOCHA Investigators. Circulation 1996; 94(11): 2807-2816. 
[142] Xiao RP, Tomhave ED, Wang DJ, et al. Age-associated reductions in cardiac beta1- and beta2-adrenergic responses without changes in inhibitory $\mathrm{G}$ proteins or receptor kinases. $\mathrm{J}$ Clin Invest 1998; 101(6): 1273-1282.

[143] Brodde OE, Michel MC, Zerkowski H-R. Signal transduction mechanisms controlling cardiac contractility and their alterations in chronic heart failure. Cardiovasc Res 1995; 30(4): $570-584$

[144] Grimm M, Gsell S, Mittmann C, et al. Inactivation of Gia proteins increases arrhythmogenic effects of $\beta$-adrenergic stimulation in the heart. J Mol Cell Cardiol 1998; 30(10): 1917-1928.

[145] Eschenhagen T, Mende U, Diederich M, et al. Chronic treatment with carbachol sensitizes the myocardium to cAMP-induced arrhythmia. Circulation 1996; 93(4): 763-771.

[146] Tumer N, Houck WT, Roberts J. Upregulation of adrenergic beta receptor subtypes in the senescent rat heart. Mech Ageing Dev 1989; 49(3): 235-243.

[147] Ungerer M, Böhm M, Elce JS, et al. Altered expression of $\beta$-adrenergic receptor kinase and $\beta_{1}$-adrenergic receptors in the failing human heart. Circulation 1993; 87(2): 454-463.

[148] Kiuchi K, Shannon RP, Komamura K, et al. Myocardial beta-adrenergic receptor function during the development of pacing-induced heart failure. J Clin. Invest 1993; 91(3): 907-914.

[149] Bristow MR, Minobe WA, Raynolds MV, et al. Reduced beta 1 receptor messenger RNA abundance in the failing human heart. J Clin. Invest 1993; 92(6): 2737-2745.

[150] Cerbai E, Guerra L, Varani K, et al. Beta-adrenoceptor subtypes in young and old rat ventricular myocytes: a combined patch-clamp and binding study. Br J Pharmacol 1995; 116(2): 1835-1842.

[151] White M, Roden R, Minobe $\mathrm{W}$, et al. Age-related changes in beta-adrenergic neuroeffecter systems in the human heart. Circulation 1994; 90(3): 1225-1238.

[152] Böhm M, Dorner H, Htun P, et al. Effects of exercise on myocardial adenylate cyclase and Gia expression in senescence. Am J Physiol 1993; 264(3 Pt 2): H805-H814.

[153] Chin JH, Hiremath AN, Hoffman BB. cAMP signaling mechanisms with aging in rats. Mech Ageing Dev 1996; 86(1): 11-26.

[154] Feldman AM. Modulation of adrenergic receptors and G-transduction proteins in failing human ventricular myocardium. Circulation 1993; 87(5 Suppl): IV27-IV34.

[155] Eschenhagen T, Mende U, Nose M, et al. Increased messenger RNA level of the inhibitory G protein $a$ subunit Gia-2 in human endstage heart failure. Circ Res 1992; 70(4): 688-696. 
[156] Lohse MJ, Engelhardt S, Eschenhagen T. What is the role of the $\beta$-adrenergic signaling in heart failure?. Circ Res 2003; 93(10): 896-906.

[157] Penela P, Murga C, Ribas C, et al. Mechanism of regulation of G protein-coupled receptor kinases (GRKs) and cardiovascular disease. Cardiovasc Res 2006; 69(1): 46-56.

[158] Hata JA, Williams ML, Koch WJ. Genetic manipulation of myocardial $\beta$-adrenergic receptor activation and desensitization. J Mol Cell Cardiol 2004; 37(1): 11-21.

[159] Leineweber K, Rohe P, Beilfuss A, et al. G-Protein-coupled receptor kinase activity in human heart failure:effects of $\beta$-adrenoceptor blockade. Cardiovasc Res 2005; 66(3): 512-519. [160] Iaccarino G, Koch WJ. Therapeutic potential of G protein coupled receptor kinases in the heart. Expert Opin Investig Drugs 1999; 8(5): 545-554.

[161] Hata JA, Koch WJ. Phosphorylation of G-protein-coupled receptors: GPCR kinases in heart disease. Mol Interv 2003; 3(5): 264-272.

[162] Leineweber K, Klapproth S, Beilfuss A, et al. Unchanged G-protein-coupled receptor kinase

activity in the aging human heart. J Am Coll Cardiol 2003; 42(8): 1487-1492.

[163] Liberman M. Obesity and insulin resistance: "window" to myocardial dysfunction. Arq Bras Cardiol 2011; 97(2): 92-93.

[164] Scaglione R, Dichiara MA, Indovina A, et al. Left ventricular diastolic and systolic function in normotensive obese subjects: influence of degree and duration of obesity. Eur Heart J 1992; 13(6): 738-742.

[165] Schmieder RE, Messerli FH. Obesity hypertension. Med Clin North Am 1987; 71(5): 991-1001.

[166] Lavie CJ, Messerli FH. Cardiovascular adaptation to obesity and hypertension. Chest 1986; 90(2): 275-279.

[167] de Divitiis O, Fazio S, Petitto M, et al. Obesity and cardiac function. Circulation 1981; 64(3): 477- 482.

[168] Lauer MS, Anderson KM, Kannel WB, et al. The impact of obesity on left ventricular mass and geometry. The Framingham Heart Study. JAMA 1991; 266(2): 231-236.

[169] Alpert MA, Lambert CR, Terry BE, et al. Interrelationship of left ventricular mass, systolic function and diastolic filling in normotensive morbidly obese patients. Int $\mathrm{J}$ Obes Relat Metab Disord 1995; 19(8): 550-557.

[170] Peterson LR, Waggoner AD, Schechtman KB, et al. Alterations in left ventricular structure 
and function in young healthy obese women: assessment by echocardiography and tissue doppler imaging. J Am Coll Cardiol 2004; 43(8): 1399-1404.

[171] Peterson LR, Herrero P, Schechtman KB, et al. Effect of obesity and insulin resistance on myocardial substrate metabolism and efficiency in young women. Circulation 2004; 109(18): 2191-2196.

[172] Fang Y, Nicol L, Harouki N, et al. Improvement of left ventricular diastolic function induced by $\beta$-blockade: a comparison between nebivolol and metoprolol. J Mol Cell Cardiol 2011; 51(2): 168-176.

[173] Segel LD, Rendig SV, Mason DT, et al. Isolated hearts from obese rats show impaired function during hypoxia. Proc Soc Exp Biol Med 1980; 163(1): 111-119.

[174] Hohl CM, Hu B, Fertel RH, et al. Effects of obesity and hypertension on ventricular myocytes: comparison of cells from adult SHHF/Mcc-cp and JCR:LA-cp rats. Cardiovasc Res 1993; 27(2): 238-242.

[175] Lehnen AM, Rodrigues B, Irigoyen MC, et al. Cardiovascular changes in animal models of metabolic syndrome. J Diabetes Res 2013; 2013: 761314.

[176] Zhou X, Ma L, Habibi J, et al. Nebivolol improves diastolic dysfunction and myocardial remodeling through reductions in oxidative stress in the Zucker obese rat. Hypertension 2010; 55(4): 880-888.

[177] Debin R, Lauzier B, Sicard P, et al. Are Zucker obese rats a useful model for cardiovascular complications in metabolic syndrome? Physical, biochemical and oxidative stress considerations. Fundam Clin Pharmacol 2009; 23(1): 59-67.

[178] Wang P, Chatham JC. Onset of diabetes in Zucker diabetic fatty (ZDF) rats leads to improved recovery of function after ischemia in the isolated perfused heart. Am J Physiol Endocrinol Metab 2004; 286(5): E725-736.

[179] Lima-Leopoldo AP, Leopoldo AS, da Silva DC, et al. Long-term obesity promotes alterations in diastolic function induced by reduction of phospholamban phosphorylation at serine-16 without affecting calcium handling. J Appl Physiol 2014; 117(6): 669-678.

[180] Medei E, Lima-Leopoldo AP, Pereira-Junior PP, et al. Could a high-fat diet rich in unsaturated fatty acids impair the cardiovascular system?. Can J Cardiol 2010; 26(10): 542548.

[181] Smith AD, Brands MW, Wang MH, et al. Obesity-induced hypertension develops in young rats independently of the renin-angiotensin-aldosterone system. Exp Biol Med 2006; 231(3): 282-287. 
[182] Fitzgerald SM, Henegar JR, Brands MW, et al. Cardiovascular and renal responses to a high-fat diet in Osborne-Mendel rats. Am J Physiol Regul Integr Comp Physiol 2001; 281(2): R547-552.

[183] Rider OJ, Francis JM, Ali MK, et al. Determinants of left ventricular mass in obesity: a cardiovascular magnetic resonance study. J Cardiovasc Magn Reson 2009; 11: 9.

[184] Lima-Leopoldo AP, Leopoldo AS, Silva DC, et al. Influence of long-term obesity on myocardial gene expression. Arq Bras Cardiol 2013; 100(3): 229-237.

[185] Nascimento AF, Luvizotto RA, Leopoldo AS, et al. Long-term high-fat diet-induced obesity decreases the cardiac leptin receptor without apparent lipotoxicity. Life Sci 2011; 88(23-24): 1031-1038.

[186] Abel ED, Litwin SE, Sweeney G. Cardiac remodeling in obesity. Physiol Rev 2008; 88(2): 389-419.

[187] Wensley I, Salaveria K, Bulmer AC, et al. Myocardial structure, function, and ischaemic tolerance in a rodent model of obesity with insulin resistance. Exp Physiol 2013; 98(11): 1552-1564.

[188] Dhanasekaran A, Gruenloh SK, Buonaccorsi JN, et al. Multiple antiapoptotic targets of the PI3K/Akt survival pathway are activated by epoxyeicosatrienoic acids to protect cardiomyocytes from hypoxia/anoxia. Am J Physiol Heart Circ Physiol 2008; 294(2): H724735 .

[189] Fang CX, Dong F, Thomas DP, et al. Hypertrophic cardiomyopathy in high-fat dietinduced obesity: role of suppression of forkhead transcription factor and atrophy gene transcription. Am J Physiol Heart Circ Physiol 2008; 295(3): H1206-H1215.

[190] Leopoldo AS, Sugizaki MM, Lima-Leopoldo AP, et al. Cardiac remodeling in a rat model of diet-induced obesity. Can J Cardiol 2010; 26(8): 423-429.

[191] Berkalp B, Cesur V, Corapcioglu D, et al. Obesity and left ventricular diastolic dysfunction. Int J Cardiol 1995; 52(1): 23-26.

[192] Iacobellis G, Ribaudo MC, Zappaterreno A, et al. Adapted changes in left ventricular structure and function in severe uncomplicated obesity. Obes Res 2004; 12(10): 1616-1621.

[193] Iacobellis G, Ribaudo MC, Leto G, et al. Influence of excess fat on cardiac morphology and

function: study in uncomplicated obesity. Obes Res 2002; 10(8): 767-773.

[194] Jessup M, Brozena S. Heart failure. N Engl J Med 2003; 348(20): 2007-2018.

[195] Morricone L, Malavazos AE, Coman C, et al. Echocardiographic abnormalities in normotensive obese patients: relationship with visceral fat. Obes Res 2002; 10(6): 489-498. 
[196] Wikstrand J, Pettersson P, Björntorp P. Body fat distribution and left ventricular morphology and function in obese females. J Hypertens 1993; 11(11): 1259-266.

[197] Boluyt MO, Bing OH, Lakatta EG. The ageing spontaneously hypertensive rat as a model of the transition from stable compensated hypertrophy to heart failure. Eur Heart $\mathrm{J}$ 1995; 16 (Suppl N): 19-30.

[198] Davis CL, Kapuku G, Snieder H, et al. Insulin resistance syndrome and left ventricular mass in healthy young people. Am J Med Sci 2002; 324(2): 72-75.

[199] Reilly MP, Rader DJ. The metabolic syndrome: more than the sum of its parts? Circulation 2003; 108(13): 1546-1551.

[200] Webber KT, Pick R, Jalil JE, et al. Patterns of myocardial fibrosis. J Mol Cell Cardiol 1989; 21( Suppl 5): 121-131.

[201] Jain A, Avendano G, Dharamsey S, et al. Left ventricular diastolic function in hypertension and role of plasma glucose and insulin: comparison with diabetic heart. Circulation 1996; 93(7): 1396-1402.

[202] Sasson Z, Rasooly Y, Gupta R, et al. Left atrial enlargement in healthy obese: prevalence and relation to left ventricular mass and diastolic function. Can J Cardiol 1996; 12(3): $257-263$.

[203] Minhas KM, Khan SA, Raju SV, et al. Leptin repletion restores depressed \{beta\}adrenergic contractility in ob/ob mice independently of cardiac hypertrophy. J Physiol 2005; 565(Pt 2): 463-474

[204] Carroll JF, Kyser CK, Martin MM. Beta-Adrenoceptor density and adenylyl cyclase activity in obese rabbit hearts. Int J Obes Relat Metab Disord 2002; 26(5): 627-632.

[205] Chatelain P, Robberecht P, De Neef P, et al. Impairment of hormone-stimulated cardiac adenylate cyclase activity in the genetically obese (fa/fa) Zucker rat. Pflügers Arch 1981; 390(1): 10-16.

[206] Zou Y, Komuro I, Yamazaki T, et al. Both Gs and Gi proteins are critically involved in isoproterenol-induced cardiomyocyte hypertrophy. J Biol Chem 1999; 274(14): 9760-9770.

[207] Seifert R, Wenzel-Seifert K, Lee TW, et al. Different effects of Gsalpha splice variants on beta2-adrenoreceptor-mediated signaling. The beta2-adrenoreceptor coupled to the long splice variant of Gsalpha has properties of a constitutively active receptor. J Biol Chem 1998; 273(18): 5109-5116.

[208] Takizawa T, Arai M, Yoguchi A, et al. Transcription of the SERCA2 gene is decreased in pressure-overloaded hearts: A study using in vivo direct gene transfer into living myocardium. J Mol Cell Cardiol 1999; 31(12): 2167-2174. 
[209] Gupta MP. Factors controlling cardiac myosin-isoform shift during hypertrophy and heart failure. J Mol Cell Cardiol 2007; 43(4): 388-403.

[210] Regan TJ, Lyons MM, Ahmed SS, et al. Evidence for cardiomyopathy in familial diabetes mellitus. J Clin Invest 1977; 60(4): 884-899.

[211] Fein FS. Diabetic cardiomyopathy. Diabetes Care 1990; 13(11): 1169-1179.

[212] Bell DS. Diabetic cardiomyopathy. Diabetes Care 2003; 26(10): 2949-2951.

[213] Di Bonito P, Moio N, Cavuto L, et al. Early detection of diabetic cardiomyopathy: usefulness of tissue Doppler imaging. Diabet Med 2005; 22(12): 1720-1725.

[214] Rakowski H, Appleton C, Chan KL, et al. Canadian consensus recommendations for the measurement and reporting of diastolic dysfunction by echocardiography: from the Investigators

of Consensus on Diastolic Dysfunction by Echocardiography. J Am Soc Echocardiogr 1996; 9(5): 736-760.

[215] Poornima IG, Parikh P, Shannon RP. Diabetic cardiomyopathy: the search for a unifying hypothesis. Circ Res 2006; 98(5): 596-605.

[216] Forbes JM, Cooper ME. Mechanisms of diabetic complications. Physiol Rev 2013; 93(1): 137-188.

[217] Howarth FC, Qureshi MA, Hassan Z, et al. Contractility of ventricular myocytes is well preserved despite altered mechanisms of $\mathrm{Ca} 2+$ transport and a changing pattern of mRNA in aged type 2 Zucker diabetic fatty rat heart. Mol Cell Biochem 2012; 361(1-2): 267-280.

[218] von Bibra H, Thrainsdottir IS, Hansen A, et al. Tissue Doppler imaging for the detection and quantitation of myocardial dysfunction in patients with type 2 diabetes mellitus. Diabetes Vasc Dis Res 2005; 2(1): 24-30.

[219] Sharman JE, Haluska BA, Fang ZY, et al. Association of arterial wave properties and diastolic dysfunction in patients with type 2 diabetes mellitus. Am J Cardiol 2007; 99(6): 844848.

[220] Dounis V, Siegmund T, Hansen A, et al. Global myocardial perfusion and diastolic function are impaired to a similar extent in patients with type 2 diabetes mellitus and in patients with coronary artery disease-evaluation by contrast echocardiography and pulsed tissue Doppler. Diabetologia 2006; 49(11): 2729-2740.

[221] Markuszewski L, Grycewicz T, Pietruszynski R, et al. Glycosylated hemoglobin and left ventricular diastolic dysfunction in patients with type 2 diabetes mellitus. Pol Merkur Lek 2006; 21(121): 8-11. 
[222] Song D, Kuo KH, Yao R, et al. Inducible nitric oxide synthase depresses cardiac contractile function in Zucker diabetic fatty rats. Eur J Pharmacol 2008; 579(1-3): 253-259.

[223] Nielsen LB, Bartels ED, Bollano E. Overexpression of apolipoprotein B in the heart impedes cardiac triglyceride accumulation and development of cardiac dysfunction in diabetic mice. J Biol Chem 2002; 277(30): 27014-27020.

[224] Suarez J, Scott B, Dillmann WH. Conditional increase in SERCA2a protein is able to reverse contractile dysfunction and abnormal calcium flux in established diabetic cardiomyopathy. Am J Physiol Regul Integr Comp Physiol 2008; 295(5): R1439-1445.

[225] Kajstura J, Fiordaliso F, Andreoli AM, et al. IGF-1 overexpression inhibits the development of diabetic cardiomyopathy and angiotensin II-mediated oxidative stress. Diabetes 2001; 50(6): 1414-1424.

[226] Van Linthout S, Seeland U, Riad A, et al. Reduced MMP-2 activity contributes to cardiac fibrosis in experimental diabetic cardiomyopathy. Basic Res Cardiol 2008; 103(4): 319-327.

[227] Bugger H, Abel ED. Rodent models of diabetic cardiomyopathy. Dis Model Mech 2009; 2(9-10): 454-466.

[228] Lacombe VA, Viatchenko-Karpinski S, Terentyev D, et al. Mechanisms of impaired calcium handling underlying subclinical diastolic dysfunction in diabetes. Am J Physiol Regul Integr Comp Physiol 2007; 293(5): R1787-1797.

[229] Christoffersen C, Bollano E, Lindegaard ML, et al. Cardiac lipid accumulation associated with diastolic dysfunction in obese mice. Endocrinology 2003; 144(8): 3483-3490. [230] Stuckey DJ, Carr CA, Tyler DJ, et al. Novel MRI method to detect altered left ventricular ejection and filling patterns in rodent models of disease. Magn Reson Med 2008; 60(3): 582-587.

[231] Golfman LS, Wilson CR, Sharma S, et al. Activation of PPARgamma enhances myocardial glucose oxidation and improves contractile function in isolated working hearts of ZDF rats. Am J Physiol Endocrinol Metab 2005; 289(2): E328-336.

[232] Barouch LA, Berkowitz DE, Harrison RW, et al. Disruption of leptin signaling contributes to cardiac hypertrophy independently of body weight in mice. Circulation 2003; 108(6): 754-759.

[233] Pereira L, Matthes J, Schuster I, et al. Mechanisms of [Ca2+]i transient decrease in cardiomyopathy of db/db type 2 diabetic mice. Diabetes 2006; 55(3): 608-615.

[234] Howarth FC, Qureshi MA. Myofilament sensitivity to $\mathrm{Ca}^{2+}$ in ventricular myocytes from the Goto-Kakizaki diabetic rat. Mol Cell Biochem 2008; 315(1-2): 69-74. 
[235] Howarth FC, Jacobson M, Shafiullah M, et al. Long-term effects of type 2 diabetes mellitus on heart rhythm in the Goto-Kakizaki rat. Exp Physiol 2008; 93(3): 362-369.

[236] El-Omar MM, Yang ZK, Phillips AO, et al. Cardiac dysfunction in the Goto-Kakizaki rat. A model of type II diabetes mellitus. Basic Res Cardiol 2004; 99(2): 133-141.

[237] Schäfer S, Huber J, Wihler C, et al. Impaired left ventricular relaxation in type 2 diabetic rats is related to myocardial accumulation of $\mathrm{N}(1)$ - (carboxymethyl) lysine. Eur $\mathrm{J}$ Heart Fail 2006; 8(1): 2-6.

[238] Radovits T, Korkmaz S, Loganathan S, et al. Comparative investigation of the left ventricular pressure--volume relationship in rat models of type 1 and type 2 diabetes mellitus. Am J Physiol Heart Circ Physiol 2009; 297(1): H125-H133.

[239] Baynes J, Murray DB. Cardiac and renal function are progressively impaired with aging in Zucker diabetic fatty type II diabetic rats. Oxid Med Cell Longev 2009; 2(5): 328-334.

[240] van den Brom CE, Huisman MC, Vlasblom R, et al. Altered myocardial substrate metabolism is associated with myocardial dysfunction in early diabetic cardiomyopathy in rats: studies using positron emission tomography. Cardiovasc Diabetol 2009; 8: 39.

[241] Fredersdorf S, Thumann C, Ulucan C, et al. Myocardial hypertrophy and enhanced left ventricular contractility in Zucker diabetic fatty rats. Cardiovasc Pathol 2004; 13(1): 11-19.

[242] Wang P, Lloyd SG, Zeng H, et al. Impact of altered substrate utilization on cardiac function in isolated hearts from Zucker diabetic fatty rats. Am J Physiol Heart Circ Physiol 2005; 288(5): H2102-H2110.

[243] Zhou YT, Grayburn P, Karim A, et al. Lipotoxic heart disease in obese rats: implications for human obesity. Proc Natl Acad Sci U S A 2000; 97(4): 1784-1789.

[244] Daniels A, Linz D, van Bilsen M, et al. Long-term severe diabetes only leads to mild cardiac diastolic dysfunction in Zucker diabetic fatty rats. Eur J Heart Fail 2012; 14(2): 193 201.

[245] van den Brom CE, Bosmans JW, Vlasblom R, et al. Diabetic cardiomyopathy in Zucker diabetic fatty rats: the forgotten right ventricle. Cardiovasc Diabetol 2010; 9: 25.

[246] Mittal SR. Right ventricular functions in patients with type 2 diabetes below 50 years. J Assoc Physicians India 2007; 55: 599-600.

[247] Movahed MR, Milne N. Presence of biventricular dysfunction in patients with type II diabetes mellitus. Congest Heart Fail 2007; 13(2): 78-80.

[248] Kosmala W, Przewlocka-Kosmala M, Mazurek W. Subclinical right ventricular dysfunction in diabetes mellitus--an ultrasonic strain/strain rate study. Diabet Med 2007; 24(6): 656-663. 
[249] Scognamiglio R, Avogaro A, Casara D, et al. Myocardial dysfunction and adrenergic cardiac innervation in patients with insulin-dependent diabetes mellitus. J Am Coll Cardiol 1998; 31(2): 404-412.

[250] Altan VM, Arioglu E, Guner S, et al. The influence of diabetes on cardiac betaadrenoceptor subtypes. Heart Fail Rev 2007; 12(1): 58-65.

[251] Bidasee KR, Zheng $\mathrm{H}$, Shao $\mathrm{CH}$, et al. Exercise training initiated after the onset of diabetes preserves myocardial function: effects on expression of beta-adrenoceptors. J Appl Physiol 2008; 105(3): 907-914.

[252] Gando S, Hattori Y, Akaishi Y, et al. Impaired contractile response to beta adrenoceptor stimulation in diabetic rat hearts: alterations in beta adrenoceptors- $G$ protein-adenylate cyclase system and phospholamban phosphorylation. J Pharmacol Exp Ther 1997; 282(1): 475-484.

[253] Amour J, David JS, Vivien B, et al. Interaction of halogenated anesthetics with alphaand beta-adrenoceptor stimulations in diabetic rat myocardium. Anesthesiology 2004; 101(5): 1145-1152.

[254] Malhotra A, Penpargkul S, Fein FS, et al. The effect of streptozotocin-induced diabetes in rats on cardiac contractile proteins. Circ Res 1981; 49(6): 1243-1250.

[255] Gauthier C, Tavernier G, Charpentier F, et al. Functional beta3-adrenoceptor in the human heart. J Clin Invest 1996; 98(2): 556-562.

[256] Smith JM, Paulson DJ, Romano FD. Inhibition of nitric oxide synthase by L-NAME improves ventricular performance in streptozotocin-diabetic rats. J Mol Cell Cardiol 1997; 29(9): 2393-2402.

[257] Huisamen B, Marais E, Genade S, et al. Serial changes in the myocardial betaadrenergic signaling system in two models of non-insulin dependent diabetes mellitus. Mol Cell Biochem 2001; 219(1-2): 73-82.

[258] Heyliger CE, Pierce GN, Singal PK, et al. Cardiac alpha- and beta-adrenergic receptor alterations in diabetic cardiomyopathy. Basic Res Cardiol 1982; 77(6): 610-618.

[259] Dinçer UD, Bidasee KR, Güner S, et al. The effect of diabetes on expression of beta1-, beta2-, and beta3-adrenoreceptors in rat hearts. Diabetes 2001; 50(2): 455-461.

[260] Amour J, Kersten JR. Diabetic cardiomyopathy and anesthesia: bench to bedside. Anesthesiology 2008; 108(3): 524-530.

[261] Choi KM, Zhong Y, Hoit BD, et al. Defective intracellular $\mathrm{Ca}^{2+}$ signaling contributes to cardiomyopathy in Type 1 diabetic rats. Am J Physiol Heart Circ Physiol 2002; 283(4): H1398-H1408. 
[262] Setién R, Alday A, Diaz-Asensio C, et al. Mechanisms responsible for the trophic effect of beta-adrenoceptors on the I(to) current density in type 1 diabetic rat cardiomyocytes. Cell Physiol Biochem 2013; 31(1): 25-36.

[263] Gøtzsche O. Myocardial cell dysfunction in diabetes mellitus. A review of clinical and experimental studies. Diabetes 1986; 35(10): 1158-1162.

[264] Gøtzsche O. The adrenergic beta-receptor adenylate cyclase system in heart and lymphocytes from streptozotocin-diabetic rats. In vivo and in vitro evidence for a desensitized myocardial beta-receptor. Diabetes 1983; 32(12): 1110-1116.

[265] Netticadan T, Temsah RM, Kent A, et al. Depressed levels of Ca2+-cycling proteins may underlie sarcoplasmic reticulum dysfunction in the diabetic heart. Diabetes 2001; 50(9): 2133-2138.

[266] Belke DD, Dillmann WH. Altered cardiac calcium handling in diabetes. Curr Hypertens Rep 2004; 6(6): 424-429.

[267] Wold LE, Dutta K, Mason MM, et al. Impaired SERCA function contributes to cardiomyocyte dysfunction in insulin resistant rats. J Mol Cell Cardiol 2005; 39(2): 297-307. [268] Abe T, Ohga Y, Tabayashi N, et al. Left ventricular diastolic dysfunction in type 2 diabetes mellitus model rats. Am J Heart Circ Physiol 2002; 282(1): H139-H148.

[269] Yagi K, Kim S, Wanibuchi H, et al. Characteristics of diabetes, blood pressure, and cardiac and renal complications in Otsuka Long-Evans Tokushima Fatty rats. Hypertension 1997; 29(3): 728-735.

[270] Li SY, Yang X, Ceylan-Isik AF, et al. Cardiac contractile dysfunction in Lep/Lep obesity is accompanied by NADPH oxidase activation, oxidative modification of sarco(endo)plasmic reticulum Ca2+-ATPase and myosin heavy chain isozyme switch. Diabetologia 2006; 49(6): 1434-1446.

[271] Howarth FC, Qureshi MA, Hassan Z, et al. Changing pattern of gene expression is associated with ventricular myocyte dysfunction and altered mechanisms of $\mathrm{Ca}^{2+}$ signaling in young type 2 Zucker diabetic fatty rat heart. Exp Physiol 2011; 96(3): 325-337.

[272] Fredersdorf S, Thumann C, Zimmermann WH, et al. Increased myocardial SERCA expression in early type 2 diabetes mellitus is insulin dependent: In vivo and in vitro data. Cardiovasc Diabetol 2012; 11:57.

[273] Riou B, Lecarpentier Y, Viars P. Inotropic effect of ketamine on rat cardiac papillary muscle. Anesthesiology 1989; 71(1): 116-125.

[274] David JS, Tavernier B, Amour J, et al. Myocardial effects of halothane and sevoflurane in diabetic rats. Anesthesiology 2004; 100(5): 1179-1187. 
[275] Hanouz JL, Riou B, Massias L, et al. Interaction of halothane with alpha- and betaadrenoceptor stimulations in rat myocardium. Anesthesiology 1997; 86(1): 147-159.

[276] Hanouz JL, Vivien B, Gueugniaud PY, et al. Interaction of isoflurane and sevoflurane with alpha- and beta-adrenoceptor stimulations in rat myocardium. Anesthesiology 1998; 88(5): 1249-1258.

[277] David JS, Vivien B, Lecarpentier Y, et al. Extracellular calcium modulates the effects of protamine on rat myocardium. Anesth Analg 2001; 92(4): 817-823.

[278] Aleixandre de Artiñano A, Miguel Castro M. Experimental rat models to study the metabolic syndrome. Br J Nutr 2009; 102(9): 1246-1253.

[279] Burton-Freeman B. Dietary fiber and energy regulation. J Nutr 2000; 130(2S Suppl): 272S-275S.

[280] An D, Rodrigues B. Role of changes in cardiac metabolism in development of diabetic cardiomyopathy. Am J Physiol Heart Circ Physiol 2006; 291(4): H1489-H1506.

[281] Bugger H, Abel ED. Molecular mechanisms for myocardial mitochondrial dysfunction in the metabolic syndrome. Clin Sci (Lond) 2008; 114(3): 195-210.

[282] Boustany-Kari CM, Gong M, Akers WS, et al. Enhanced vascular contractility and diminished coronary artery flow in rats made hypertensive from diet-induced obesity. Int $\mathbf{J}$ Obes (Lond) 2007; 31(11): 1652-1659.

[283] Ghibaudi L, Cook J, Farley C, et al. Fat intake affects adiposity, comorbidity factors, and energy metabolism of sprague-dawley rats. Obes Res 2002; 10(9): 956-963.

[284] Buettner R, Parhofer KG, Woenckhaus M, et al. Defining high-fat-diet rat models: metabolic and molecular effects of different fat types. J Mol Endocrinol 2006; 36(3): 485-501. [285] Kanasaki K, Koya D. Biology of obesity: lessons from animal models of obesity. J Biomed Biotechnol 2011; 2011: 197636.

[286] Chua SC Jr, Chung WK, Wupeng XS, et al. Phenotypes of mouse diabetes and rat fatty due to mutations in the OB (leptin) receptor. Science 1996; 271(5251): 994-996.

[287] Chua SC Jr, White DW, Wu-Peng XS, et al. Phenotype of fatty due to Gln269Pro mutation in the leptin receptor (Lepr). Diabetes 1996; 45(8): 1141-1143.

[288] Phillips MS, Liu Q, Hammond HA, et al. Leptin receptor missense mutation in the fatty Zucker rat. Nature Genet 1996; 13(1): 18-19.

[289] Zhang Y, Proenca R, Maffei M, et al. Positional cloning of the mouse obese gene and its human homologue. Nature 1994; 372(6505): 425-432.

[290] Ahima RS, Flier JS. Leptin. Annu Rev Physiol 2000; 62: 413-437. 
[291] Himms-Hagen J. Physiological roles of the leptin endocrine system; differences between mice and humans. Crit Rev Clin Lab Sci 1999; 36(6): 575-655.

[292] Palou A, Serra F, Bonet ML, et al. Obesity: molecular bases of a multifactorial problem. Eur J Nutr 2000; 39(4): 127-144.

[293] Enriori PJ, Evans AE, Sinnayah P, et al. Diet-induced obesity causes severe but reversible leptin resistance in arcuate melanocortin neurons. Cell Metab 2007; 5(3): 181-194.

[294] Münzberg H, Flier JS, Bjørbaek C. Region-specific leptin resistance within the hypothalamus of diet-induced obese mice. Endocrinology 2004; 145(11): 4880-4889.

[295] Wang B, Chandrasekera PC, Pippin JJ. Leptin- and leptin receptor-deficient rodent models: relevance for human type 2 diabetes. Curr Diabetes Rev 2014; 10(2): 131-145.

[296] Hardie LJ, Rayner DV, Holmes S, et al. Circulating leptin levels are modulated by fasting, cold exposure and insulin administration in lean but not Zucker ( fa/fa) rats as measured by ELISA. Biochem Biophys Res Commun 1996; 223(3): 660-665.

[297] Picó C, Sánchez J, Oliver P, et al. Leptin production by the stomach is up-regulated in obese ( fa/fa) Zucker rats. Obes Res 2002; 10(9): 932-938.

[298] Beck B. Neuropeptides and obesity. Nutrition 2000; 16(10): 916-923.

[299] Beck B, Burlet A, Nicolas JP, et al. Hyperphagia in obesity is associated with a central peptidergic dysregulation in rats. J Nutr 1990; 120(7): 806-811.

[300] Beck B, Burlet A, Nicolas JP, et al. Galanin in the hypothalamus of fed and fasted lean and obese Zucker rats. Brain Res 1993; 623(1): 124-130.

[301] Stricker-Krongrad A, Dimitrov T, Beck B. Central and peripheral dysregulation of melanin-concentrating hormone in obese Zucker rats. Brain Res Mol Brain Res 2001; 92(1-2): $43-48$

[302] Stern JS, Johnson PR. Spontaneous activity and adipose cellularity in the genetically obese Zucker rat (fafa). Metabolism 1977; 26(4): 371-380.

[303] Zucker TF, Zucker LM. Hereditary obesity in the rat associated with high serum fat and cholesterol. Proc Soc Exp Biol Med 1962; 110(1): 165-171.

[304] Zucker TF, Zucker LM. Fat accretion and growth in the rat. J Nutr 1963; 80(1): 6-19.

[305] Johnson PR, Zucker LM, Cruce JA, et al. Cellularity of adipose depots in the genetically obese Zucker rat. J Lipid Res 1971; 12(6): 706-714.

[306] Stern J, Johnson PR, Greenwood MRC, et al. Insulin resistance and pancreatic insulin release in the genetically obese Zucker rat. Proc Soc Exp Biol Med 1972; 139(1): 66-69.

[307] Bryce GF, Johnson PR, Sullivan AC, et al. Insulin and glucagon: plasma levels and pancreatic release in the genetically obese Zucker rat. Horm Met Res 1977; 9(5): 366-370. 
[308] Ionescu E, Sauter JF, Jeanrenaud B. Abnormal glucose tolerance in genetically obese (fa/fa) rats. Am J Physiol 1985; 248(5 Pt 1): E500-E506.

[309] Muller S, Cleary MP. Glucose metabolism in isolated adipocytes from ad libitum- and restricted-fed lean and obese Zucker rats at two different ages. Proc Soc Exp Biol Med 1988; 187(4): 398-407.

[310] Kasiske BL, O’Donnell MP, Keane WF. The Zucker rat model of obesity, insulin resistance, hyperlipidemia, and renal injury. Hypertension 1992; 19(1 Suppl): I110-I115.

[311] Augstein P, Salzsieder E. Morphology of pancreatic islets: a time course of pre-diabetes in Zucker fatty rats. Methods Mol Biol 2009; 560: 159-189.

[312] Maggio CA, Greenwood MR. Adipose tissue lipoprotein lipase (LPL) and triglyceride uptake in Zucker rats. Physiol Behav 1982; 29(6): 1147-1152.

[313] Gruen RK, Hietanen E, Greenwood MR. Increased adipose tissue lipoprotein lipase activity during the development of the genetically obese rat (fa/fa). Metabolism 1978; 27(12 Suppl 2): 1955-1966.

[314] Lin RC. Serum cholesterol, lecithin-cholesterol acyltransferase, and hepatic hydroxymethilglutaryl coenzyme A reductase activities of lean and obese Zucker rats. Metabolism 1985; 34(1): 19-24.

[315] Lash JM, Sherman WM, Hamlin RL. Capillary basement membrane thickness and capillary density in sedentary and trained obese Zucker rats. Diabetes 1989; 38(7): 854-860.

[316] Ernsberger P, Nelson DO. Refeeding hypertension in dietary obesity. Am J Physiol 1988; 254(1 Pt 2): R47-R55.

[317] Koletsky S. Pathologic findings and laboratory data in a new strain of obese hypertensive rats. Am J Pathol 1975; 80(1): 129-140.

[318] Zemel MB, Sowers JR, Shehin S, et al. Impaired calcium metabolism associated with hypertension in Zucker obese rats. Metabolism 1990; 39(7): 704-708.

[319] Kurtz TW, Morris RC, Pershadsingh HA. The Zucker fatty rat as a genetic model of obesity and hypertension. Hypertension 1989; $13(6$ Pt 2): 896-901.

[320] Kasiske BL, Cleary MP, O’Donnell MP, et al. Effects of genetic obesity on renal structure and function in the Zucker rat. J Lab Clin Med 1985; 106(5): 598-604.

[321] Wu X, Mäkynen H, Kähönen M, et al. Mesenteric arterial function in vitro in three models of experimental hypertension. J Hypertens 1996; 14(3): 365-372.

[322] Yuen VG, Pederson RA, Dai S, et al. Effects of low and high dose administration of bis(maltolato)oxovanadium(IV) on fa/fa Zucker rats. Can J Physiol Pharmacol 1996; 74(9): 1001-1009. 
[323] Arvola P, Wu X, Kähönen M, et al. Exercise enhances vasorelaxation in experimental obesity associated hypertension. Cardiovasc Res 1999; 43(4): 992-1002.

[324] He Y, MacLeod KM. Modulation of noradrenalineinduced vasoconstriction in isolated perfused mesenteric arterial beds from obese Zucker rats in the presence and absence of insulin. Can J Physiol Pharmacol 2002; 80(3): 171-179.

[325] Zanchi A, Delacrétaz E, Taleb V, et al. Endothelial function of the mesenteric arteriole and mechanical behavior of the carotid artery in rats with insulin resistance and hypercholesterolaemia. J Hypertens 1995; 13(12 Pt 1): 1463-1470.

[326] Turner NC, White P. Effects of streptozotocininduced diabetes on vascular reactivity in genetically hyperinsulinaemic obese Zucker rats. J Cardiovasc Pharmacol 1996; 27(6): 884890.

[327] Alonso-Galicia M, Brands MW, Zappe DH, et al. Hypertension in obese Zucker rats. Role of angiotensin II and adrenergic activity. Hypertension 1996; 28(6): 1047-1054.

[328] Dzau VJ. Molecular and physiological aspects of tissue renin-angiotensin system: emphasis on cardiovascular control. J Hypertens Suppl 1988; 6(3): S7-S12.

[329] Unger T, Gohlke P. Tissue renin-angiotensin systems in the heart and vasculature: possible involvement in the cardiovascular actions of converting enzyme inhibitors. Am J Cardiol 1990; 65(19): 3I-10I.

[330] De Gasparo M. AT(1) and AT(2) angiotensin II receptors: key features. Drugs 2002; 62 Spec No 1: 1-10.

[331] Vincent HK, Taylor AG. Biomarkers and potential mechanisms of obesityinduced oxidant stress in humans. Int J Obes (Lond) 2006; 30(3): 400-418.

[332] Aprikian O, Busserolles J, Manach C, et al. Lyophilized apple counteracts the development of hypercholesterolemia, oxidative stress, and renal dysfunction in obese Zucker rats. J Nutr 2002; 132(7):1969-1976.

[333] Blakely S, Herbert A, Collins M, et al. Lutein interacts with ascorbic acid more frequently than with $\{$ alpha $\}$-tocopherol to alter biomarkers of oxidative stress in female Zucker obese rats. J Nutr 2003; 133(9): 2838-2844.

[334] Oltman CL, Richou LL, Davidson EP, et al. Progression of coronary and mesenteric vascular dysfunction in Zucker obese and Zucker diabetic fatty rats. Am J Physiol Heart Circ Physiol 2006; 291(4): H1780-H1787.

[335] Soltys K, Dikdan G, Koneru B. Oxidative stress in fatty livers of obese Zucker rats: rapid amelioration and improved tolerance to warm ischemia with tocopherol. Hepatology 2001; 34(1): 13-18. 
[336] Gustavsson C, Soga T, Wahlstrom E, et al. Sex-dependent hepatic transcripts and metabolites in the development of glucose intolerance and insulin resistance in Zucker diabetic fatty rats. J Mol Endocrinol 2011; 47(2): 129-143.

[337] Corsetti JP, Sparks JD, Peterson RG, et al. Effect of dietary fat on the development of non-insulin dependent diabetes mellitus in obese Zucker diabetic fatty male and female rats. Atherosclerosis 2000; 148(2): 231-241.

[338] Veniant MM, LeBel CP. Leptin: from animals to humans. Curr Pharm 2003; 9(10): 811-818.

[339] Paulsen SJ, Vrang N, Larsen LK, et al. Stereological assessment of pancreatic beta-cell mass development in male Zucker Diabetic Fatty (ZDF) rats: correlation with pancreatic betacell function. J Anat 2010; 217(5): 624-630.

[340] Mizuno M, Sada T, Kato M, et al. The effect of angiotensin II receptor blockade on an end-stage renal failure model of type 2 diabetes. J Cardiovasc Pharmacol 2006; 48(4): 135 142.

[341] Brussee V, Guo G, Dong Y, et al. Distal degenerative sensory neuropathy in a long term type 2 diabetes rat model. Diabetes 2008; 57(6): 1664-1673.

[342] Reaven GM, Chang H, Hoffman BB, et al. Resistance to insulin-stimulated glucose uptake in adipocytes isolated from spontaneously hypertensive rats. Diabetes 1989; 38(9): 1155-1160.

[343] Kvetnanský R, Rusnák M, Gasperíková D, et al. Hyperinsulinemia and sympathoadrenal system activity in the rat. Ann N Y Acad Sci 1997; 827: 118-134.

[344] Koletsky S. Obese spontaneously hypertensive rats - a model for study of atherosclerosis. Exp Mol Pathol 1973; 19(1): 53-60.

[345] Kastin AJ, Pan W, Maness LM, et al. Decreased transport of leptin across the bloodbrain barrier in rats lacking the short form of the leptin receptor. Peptides 1999; 20(12): 14491453.

[346] Michaelis OE 4th, Ellwood KC, Judge JM, et al. Effect of dietary sucrose on the SHR/N-corpulent rat: a new model for insulin-independent diabetes. Am J Clin Nutr 1984; 39(4): 612-618.

[347] Velasque MT, Bhathena SJ, Hansen CT. Leptin and its relation to obesity and insulin in the SHR/N-corpulent rat, a model of type II diabetes mellitus. Int J Exp Diabetes Res 2001; 2(3): 217-223. 
[348] Brindley DN, Russell JC. Animal models of insulin resistance and cardiovascular disease: some therapeutic approaches using JCR: LA-cp rat. Diabetes Obes Metab 2002; 4(1): $1-10$.

[349] Atgie C, Hadj-Sassi A, Bukowiecki L, et al. High lipolytic activity and dyslipidemia in a spontaneous hypertensive/NIH corpulent (SHR/N-cp) rat: a genetic model of obesity and type 2 diabetes mellitus. J Physiol Biochem 2009; 65(1): 33-41.

[350] Michaelis OE 4th, Hansen CT. The spontaneous hypertensive/NIH-corpulent rat: a new rodent model for the study of non-insulin-dependent diabetes mellitus and its complications. ILAR J 1990; 32(3): 19-22.

[351] Voyles NR, Powell AM, Timmers KI, et al. Reversible impairment of glucose-induced insulin secretion in SHR/N-cp rats. Genetic model of type II diabetes. Diabetes 1988; 37(4): 398-404.

[352] Wexler BC, Iams SG, McMurtry JP. Pathophysiological differences between obese and non-obese spontaneously hypertensive rats. Br J Exp Pathol 1980; 61(2): 195-207.

[353] Hiraoka J, Hosoda K, Ogawa Y, et al. Augmentation of obese (ob) gene expression and leptin secretion in obese spontaneously hypertensive rats (obese SHR or Koletsky rats). Biochem Biophys Res Commun 1997; 231(3): 582-585.

[354] McCune SA, Baker PB, Stills HF. SHHF/Mcc-cp rat: model of obesity, non-insulindependent diabetes, and congestive heart failure. ILAR J 1990; 32(3): 23-27.

[355] Amy RM, Dolphin PJ, Pederson RA, et al. Atherogenesis in two strains of obese rats. The fatty Zucker and LA/N-corpulent. Atherosclerosis 1988; 69(2-3): 199-209.

[356] Dolphin PJ, Amy RM, Russell JC. Effect of age on serum lipids and lipoproteins of male and female JCR: LA-corpulent rats. Biochim Biophys Acta 1990; 1042(1): 99-106.

[357] Russell JC, Shillabeer G, Bar-Tana J, et al. Development of insulin resistance in the JCR: LA-cp rat: role of triacylglycerols and effects of MEDICA 16. Diabetes 1998; 47(5): $770-778$

[358] McArthur MD, Graham SE, Russell JC, et al. Exaggerated stress-induced release of nonesterified fatty acids in JCR: LA corpulent rats. Metabolism 1998; 47(11): 1383-1390.

[359] Russell JC, Koeslag DG. JCR: LA-corpulent rat: a strain with spontaneous vascular and myocardial disease. ILAR J 1990; 32(3): 27-32.

[360] Yamori Y, Ohtaka M, Horie R, et al. Cerebral stroke and myocardial lesions in strokeprone SHR. Jpn Heart J 1978; 19(4): 609-611. 
[361] Hiraoka-Yamamoto J, Nara Y, Yasui N, et al. Establishment of a new animal model of metabolic syndrome: SHRSP fatty (fa/fa) rats. Clin Exp Pharmacol Physiol 2004; 131(1-2): 107-109.

[362] Marchesini G, Brizi M, Bianchi G, et al. Nonalcoholic fatty liver disease: a feature of the metabolic syndrome. Diabetes 2001; 50(8): 1844-1850.

[363] Filippetti R, Kloting I, Massi M, et al. Involvement of cocaine-amphetamine regulated transcript in the differential feeding responses to nociceptin/orphanin FQ in dark agouti and Wistar Ottawa Karlsburg W rats. Peptides 2007; 28(10): 1966-1973.

[364] van den Brandt J, Kovács P, Klöting I. Metabolic features in disease-resistant as well as in spontaneously hypertensive rats and newly established obese Wistar Ottawa Karlsburg inbred rats. Int J Obes Relat Metab Disord 2000; 24(12): 1618-1622.

[365] Kovács P, van den Brandt J, Klöting I. Genetic dissection of the syndrome X in the rat. Biochem Biophys Res Commun 2000; 269(3): 660-665.

[366] Klöting I, Vogt L, Serikawa T. Locus on chromosome 18 cosegregates with diabetes in the BB/OK rat subline. Diabete Metab 1995; 21(5): 338-344.

[367] van den Brandt J, Kovács P, Klöting I. Features of the metabolic syndrome in the spontaneously hypertriglyceridemic Wistar Ottawa Karlsburg W (RT1u Haplotype) rat. Metabolism 2000; 49(9): 1140-1144.

[368] Grisk O, Frauendorf T, Schlüter T, et al. Impaired coronary function in Wistar Ottawa Karlsburg W rats - a new model of the metabolic syndrome. Pflügers Arch 2007; 454(6): 1011-1021.

[369] Wisløff U, Najjar SM, Ellingsen O, et al. Cardiovascular risk factors emerge after artificial selection for low aerobic capacity. Science 2005; 307(5708): 418-420.

[370] Bonnevie-Nielsen V, Steffes MW, Lernmark A. A major loss in islet mass and B-cell function precedes hyperglycemia in mice given multiple low doses of streptozotocin. Diabetes 1981; 30(5): 424-429.

[371] Islas-Andrade S, Monsalve CR, de la Peña JE, et al. Streptozotocin and alloxan in experimental diabetes: comparison of the two models in rats. Acta Histochem Cytochem 2000; 33(3): 201-208.

[372] Bolzán AD, Bianchi MS. Genotoxicity of streptozotocin. Mutat Res 2002; 512(2-3): 121-134.

[373] Wold LE, Ren J. Streptozotocin directly impairs cardiac contractile function in isolated ventricular myocytes via a p38 map kinase-dependent oxidative stress mechanism. Biochem Biophys Res Commun 2004; 318(4): 1066-1071. 
[374] Gauguier D, Froguel P, Parent V, et al. Chromosomal mapping of genetic loci associated with non-insulin dependent diabetes in the GK rat. Nat Genet 1996; 12(1): 38-43. [375] Goto Y, Kakizaki M, Masaki N. Production of spontaneous diabetic rats by repetition of selective breeding. Tohoku J Exp Med 1976; 119(1): 85-90.

[376] Bisbis S, Bailbe D, Tormo M-A, et al. Insulin resistance in the GK rat: decreased receptor number but normal kinase activity in liver. Am J Physiol 1993; 265(5 Pt 1): E807E813.

[377] Salem KA, Adrian TE, Qureshi MA, et al. Shortening and intracellular Ca2+ in ventricular myocytes and expression of genes encoding cardiac muscle proteins in early onset type 2 diabetic Goto-Kakizaki rats. Exp Physiol 2012; 97(12): 1281-1291.

[378] Murase T, Hattori T, Ohtake M, et al. Cardiac remodeling and diastolic dysfunction in DahlS.Z-Lepr(fa)/Lepr(fa) rats: a new animal model of metabolic syndrome. Hypertens Res 2012; 35(2): 186-193.

[379] Hattori T, Murase T, Ohtake M, et al. Characterization of a new animal model of metabolic syndrome: the DahlSZ-Leprfa/Leprfa rat. Nutr Diabetes 2011; 1: e1.

[380] Friedman JM, Leibel RL, Siegel DS, et al. Molecular mapping of the mouse ob mutation. Genomics 1991; 11(4): 1054-1062.

[381] Buchanan J, Mazumder PK, Hu P, et al. Reduced cardiac efficiency and altered substrate metabolism precedes the onset of hyperglycemia and contractile dysfunction in two mouse models of insulin resistance and obesity. Endocrinology 2005; 146(12): 5341-5349.

[382] Barouch LA, Gao D, Chen L, et al. Cardiac myocyte apoptosis is associated with increased DNA damage and decreased survival in murine models of obesity. Circ Res 2006; 98(1): 119-124.

[383] Halaas JL, Gajiwala KS, Maffei M, et al. Weight-reducing effects of the plasma protein encoded by the obese gene. Science 1995; 269(5223): 543-546.

[384] Stephens TW, Basinski M, Bristow PK, et al. The role of neuropeptide $Y$ in the antiobesity action of the obese gene product. Nature 1995; 377(6549): 530-532.

[385] Pelleymounter MA, Cullen MJ, Baker MB, et al. Effects of the obese gene product on body weight regulation in ob/ob mice. Science 1995; 269(5223): 540-543.

[386] Srinivasan K, Ramarao P. Animal models in type 2 diabetes research: an overview. Indian J Med Res 2007; 125(3): 451-472.

[387] Westman S. Development of the obese-hyperglycaemic syndrome in mice. Diabetologia 1968; 4(3): 141-149. 
[388] Schwartz MW, Baskin DG, Bukowski TR, et al. Specificity of leptin action on elevated blood glucose levels and hypothalamic neuropeptide $\mathrm{Y}$ gene expression in ob/ob mice. Diabetes 1996; 45(4): 531-535.

[389] Shafrir E, Ziv E, Mosthaf L. Nutritionally induced insulin resistance and receptor defect leading to beta-cell failure in animal models. Ann N Y Acad Sci 1999; 892: 223-246.

[390] Lee AW, Cox RD. Use of mouse models in studying type 2 diabetes mellitus. Expert Rev Mol Med 2011; 13: e1.

[391] Ren J. Leptin and hyperleptinemia - from friend to foe for cardiovascular function. J Endocrinol 2004; 181(1): 1-10.

[392] Hafstad AD, Solevåg GH, Severson DL, et al. Perfused hearts from Type 2 diabetic $(\mathrm{db} / \mathrm{db})$ mice show metabolic responsiveness to insulin. Am J Physiol Heart Circ Physiol 2006; 290(5): H1763-1769.

[393] Allen TJ, Cooper ME, Lan HY. Use of genetic mouse models in the study of diabetic nephropathy. Curr Atheroscler Rep 2004; 6(3): 197-202.

[394] Han KL, Choi JS, Lee JY, et al. Therapeutic potential of peroxisome proliferators-activated receptor-alpha/gamma dual agonist with alleviation of endoplasmic reticulum stress for the treatment of diabetes. Diabetes 2008; 57(3): 737-745.

[395] Coleman DL, Hummel KP. Hyperinsulinemia in pre-weaning diabetes (db) mice. Diabetologia 1974; 10 Suppl: 607-610.

[396] Berglund O, Frankel BJ, Hellman B. Development of the insulin secretory defect in genetically diabetic (db/db) mouse. Acta Endocrinol (Copenh) 1978; 87(3): 543-551.

[397] Kashyap SR, Defronzo RA. The insulin resistance syndrome: physiological considerations. Diab Vasc Dis Res 2007; 4(1): 13-19.

[398] Nyomba BL, Ossowski VM, Bogardus C, et al. Insulin sensitive tyrosine kinase: relationship with in vivo insulin action in humans. Am J Physiol 1990; 258(6 Pt 1): E964-974. [399] Petersen KF, Shulman GI. Etiology of insulin resistance. Am J Med 2006; 119(5 Suppl 1): S10-16.

[400] Vicario P, Brady EJ, Slater EE, et al. Insulin receptor tyrosine kinase activity is unaltered in ob/ob and db/db mouse skeletal muscle membranes. Life Sci 1987; 41(10): 1233 1241.

[401] Sugizaki MM, Carvalho RF, Aragon FF, et al. Myocardial dysfunction induced by food restriction is related to morphological damage in normotensive middle-age rats. J Biomed Sci 2005; 12(4): 641-649. 
[402] Vivien B, David JS, Hanouz JL, et al. The paradoxical positive inotropic effect of sevoflurane in healthy and cardiomyopathic hamsters. Anesth Analg 2002; 95(1): 31-38.

[403] Grover SA, Kaouache M, Rempel P, et al. Years of life lost and healthy life-years lost from diabetes and cardiovascular disease in overweight and obese people: a modelling study. Lancet Diabetes Endocrinol 2015; 3(2): 114-122.

[404] Volk BM, Kunces LJ, Freidenreich DJ, et al. Effects of step-wise increases in dietary carbohydrate on circulating saturated fatty acids and palmitoleic acid in adults with metabolic syndrome. Plos One 2014; 9(11): e113605.

[405] Carroll JF, Thaden JJ, Wright AM. A comparison of two exercise training programs on cardiac responsiveness to $\beta$-stimulation in obesity. Exp Biol Med (Maywood) 2005; 230(3): 180-188.

[406] Carroll JF. Isolated heart responsiveness to beta-simulation after exercise training in obesity. Med Sci Sports Exerc 2003; 35(4): 548-554.

[407] Grassi G. Counteracting the sympathetic nervous system in essential hypertension. Curr Opin Nephrol Hypertens 2004; 13(5): 513-519.

[408] Watanabe K, Komatsu J, Kurata M, et al. Improvement of insulin resistance by troglitazone ameliorates cardiac sympathetic nervous dysfunction in patients with essential hypertension. J Hypertens 2004; 22(9): 1761-1768.

[409] Abboud I, Lerolle N, Urien S, et al. Pharmacokinetics of epinephrine in patients with septic shock: Modelization and interaction with endogenous neurohormonal status. Crit Care 2009; 13(4): R120.

[410] Beloeil H, Mazoit JX, Benhamou D, et al. Norepinephrine kinetics and dynamics in septic shock and trauma patients. Br J Anaesth 2005; 95(6):782-788.

[411] Bauman ZM, Killu KF, Rech MA, et al. Racial differences in vasopressor requirements for septic shock. Shock 2014; 41(3): 188-192.

[412] Gorski PA, Ceholski DK, Hajjar RJ. Altered myocardial calcium cycling and energetic in heart failure - a rational approach for disease treatment. Cell Metab 2015; 21(2): 183-194.

[413] Fleg JL, Strait J. Age-associated changes in cardiovascular structure and function: a fertile milieu for future disease. Heart Fail Rev 2012; 17(4-5): 545-554.

[414] Seals DR, Bell C. Chronic sympathetic activation: consequence and cause of ageassociated obesity? Diabetes 2004; 53(2): 276-284.

[415] Lakatta EG. Arterial and cardiac aging: Major shareholders in cardiovascular disease enterprises: Part III: Cellular and molecular clues to heart and arterial aging. Circulation 2003; 107(3): 490-497. 
[416] Schmidt U, del Monte F, Miyamoto MI, et al. Restoration of diastolic function in senescent rat hearts through adenoviral gene transfer of sarcoplasmic reticulum $\mathrm{Ca}(2+)$ ATPase. Circulation 2000; 101(7): 790-796. 


\section{Appendix \\ Other manuscripts published during the \\ PhD. Candidate period}

1. Jiang C, Jiang S, Zhao Y, et al. Dominant hand position improves the quality of external chest compression: A manikin study based on 2010 CPR guidelines. J Emerg Med, 2015, 48(4): 436-444.

2. Jiang C, Zhao Y. Reply to letter: Video recording and feedback of resuscitation. Resuscitation, 2012, 82(9): e181. 


\title{
Selected Topics: Prehospital Care
}

(D) CrossMark

\section{DOMINANT HAND POSITION IMPROVES THE QUALITY OF EXTERNAL CHEST COMPRESSION: A MANIKIN STUDY BASED ON 2010 CPR GUIDELINES}

\author{
Cheng Jiang, MD, ${ }^{*}{ }^{1}$ Shan Jiang, MD, ${ }^{* 1}$ Yan Zhao, MD, PHD, $†$ Bing Xu, MD, ${ }^{*}$ and Xian-long Zhou, MD* \\ ${ }^{*}$ Medical College of Wuhan University, Wuchang, Wuhan, China and †Emergency Center, Zhongnan Hospital of Wuhan University, Wuchang, \\ Wuhan, China \\ Reprint Address: Yan Zhao, MD, PHD, Emergency Center, Zhongnan Hospital of Wuhan University, 169 Donghu Road, Wuchang District, \\ Wuhan City, Hubei Province 430071, China
}

\begin{abstract}
Background: The 2010 cardiopulmonary resuscitation (CPR) guidelines increased the importance of external chest compression. However, the best hand position to be the compressing one has not been identified. Objectives: To investigate the effects of dominant or nondominant external chest compression hand position during CPR. Methods: Medical students performed five cycles of conventional CPR and completed one questionnaire. The CPR performances were manually evaluated, and detailed aspects of the external chest compression quality were assessed via the SimMan ${ }^{\circledR}$ Essential system (Laerdal China Ltd., Hangzhou, China). Results: One hundred fifty-seven students participated in the nondominant hand $(\mathrm{NH})$ group, and 68 students participated in the dominant hand (DH) group. The manual evaluations revealed no differences between the two groups. The proportion of chest compressions "above $100 \mathrm{cpm}$ [compressions per minute]" was higher in the DH group than in the $\mathrm{NH}$ group (97\% vs. $92 \%$, respectively, $p=0.002)$. The frequency distributions of the chest compression rates were also significantly different between the two groups $(p$ $<0.0001$ ). The distribution of the NH group was concentrated within "130-139" cpm, whereas this distribution was concentrated within "140-149" cpm in the DH group. The chest compression depth of the DH group was deeper than that of the NH group $(p=0.001)$. The depth of the fifth cycle was significantly decreased compared with those of cycles 1, 2, and 3 in the NH group. A greater number of full chest recoils were observed in the NH group $(p=0.02)$.
\end{abstract}

\footnotetext{
${ }^{1}$ Co-first authors.
}

Conclusion: The dominant hand position during CPR was associated with a higher chest compression rate, a greater chest compression depth, and delayed fatigue. (c) 2015 Elsevier Inc.

Keywords-cardiopulmonary resuscitation (CPR); dominant hand; chest compression; fatigue; manikin

\section{INTRODUCTION}

External chest compression is the core of cardiopulmonary resuscitation (CPR). For over 40 years, researchers have been seeking the best manipulations for increasing the quality of CPR during sudden cardiac arrest, and the International Liaison Committee on Resuscitation renews its guidelines every 5 years $(1,2)$. The American Heart Association (AHA) 2010 CPR guidelines recommend that the $\mathrm{CPR}$ sequence be changed to compression-airway-breathing (C-A-B), which increases the attention placed on the importance of chest compression (3). Moreover, the AHA also recommends "pushing hard and pushing fast."

The guidelines for external chest compression suggest "placing the heel of one hand on the center (middle) of the victim's chest and the heel of the other hand on top of the first so that the hands are overlapped and parallel" (3). Which hand should be used as the compressing hand (i.e., the lower hand that is in contact with the sternum)

RECEIVED: 10 June 2014; FinAL SUBMISSION RECEIVED: 28 September 2014;

ACCEPTED: 21 December 2014 
has not been identified. Kundra et al. showed that chest compressions are performed with fewer errors (i.e., the number of correct chest compressions is higher, and the compression depths and hand locations are better according to the 1998 European Resuscitation Council guidelines) when the dominant hand is in contact with the sternum of the Laerdal Skillmeter Resusci Anne (Laerdal China Ltd., Hangzhou, China) (4). Nikandish et al. found that positioning the dominant hand in contact with the sternum may increase the total number of the correct chest compressions during 5 min of hands-only CPR according to the European Resuscitation Council or AHA 2005 CPR guidelines when CPR is practiced on a recording Resusci Anne, but this difference was not statistically significant (5). Both of these studies were conducted based on the older CPR guidelines, which recommended that providers compress at a rate of approximately 100 compressions per minute (cpm), produce a compression depth of 40-50 mm, and that the compression to ventilation ratio be 15:2 (1998 guidelines) or 30:2 (2005 guidelines). Using an advanced manikin, the SimMan ${ }^{\circledR}$ Essential (Laerdal China Ltd.), we sought to record the parameters of CPR quality more objectively than the manikins used in the two previous studies. And meanwhile, we tried to explore whether the position of the dominant hand would influence the quality of external chest compression according to the new CPR guidelines using this objective simulation system and to learn more about the position of the dominant hand during CPR.

\section{MATERIALS AND METHODS}

\section{Participants}

All the medical students in the clinical department of our university who took a course in "Emergency Medicine" participated during the 2013/2014 academic year in their first standard CPR courses. The students had no previous experience using the SimMan Essential manikin. The study was approved by the Clinical Department of our university.

\section{Study Procedures}

As shown in Figure 1, the participants had received training in CPR skills via a formal classroom lecture that introduced the basic knowledge and skills with a PowerPoint presentation (Microsoft Corporation, Redmond, WA) and video, and repeatedly practiced their skills on a Resusci Anne (Laerdal China Ltd.) under the supervision of AHA-certificated Basic Life Support (BLS) instructors. During the courses, the instructors taught the hand placement according to the recommendations of the guidelines.
Within 1 week of the training, all participants took a CPR test that was based on the criteria of the CPR skill test in the Objective Structural Clinical Examination of our school. The participants were tested in ascending order of their student ID numbers and were presented with a simulated scenario of witnessing an adult collapse in the out-of-hospital setting. The manipulations were performed on the SimMan Essential. The participants were all asked to complete five cycles of complete conventional single-rescuer CPR on the manikin's right side of approach.

The performances of the participants were manually evaluated by an AHA-certificated BLS instructor according to the CPR Objective Structural Clinical Examination criteria (a standard template) at the scene. Each performance was exhibited as a score (the full mark of the criteria is 100). Simultaneously, two observers recorded the results on the SimMan Essential tablet personal computer remotely in another room. The computer showed objective parameters of the CPR performances. We focused on the chest compression-related parameters, including chest compression rate, chest compression depth, and the chest recoil of every cycle. The students, monitors, and observers were all blind to the aims of the study. Finally, the students were required to complete one questionnaire that included nine single-choice questions. The participants indicated their general characteristics, dominant hands, their choices of compressing hands, and self-assessments of the quality of the CPR they had given (see Appendix). During the whole procedure of the study, the organizer of the study was at the scene to monitor and observe the test flow, including the occurrence of changing hand in contact with the sternum during CPR.

Next, two researchers collected the scores, the manikin results, and the questionnaires. The participants were then divided into two groups. A nondominant hand group (NH group) included those who had used their nondominant hand as the compressing hand (i.e., the lower hand that was in contact with the sternum). A dominant hand group (DH group) included those who used their dominant hand as the compressing hand.

\section{Statistical Analyses}

The data are expressed as the means \pm the SD and numbers or percentages. Evaluations of the distributions of the variables were performed using Levene's test for homogeneity of variances. Comparisons of two means were performed using $t$-tests, comparisons of several means were performed using analyses of variance and least-significant difference tests, and comparisons of several percentages were performed using chi-squared tests or Fisher's exact tests, as appropriate. Statistical analyses were performed using SPSS software (SPSS 13.0, 


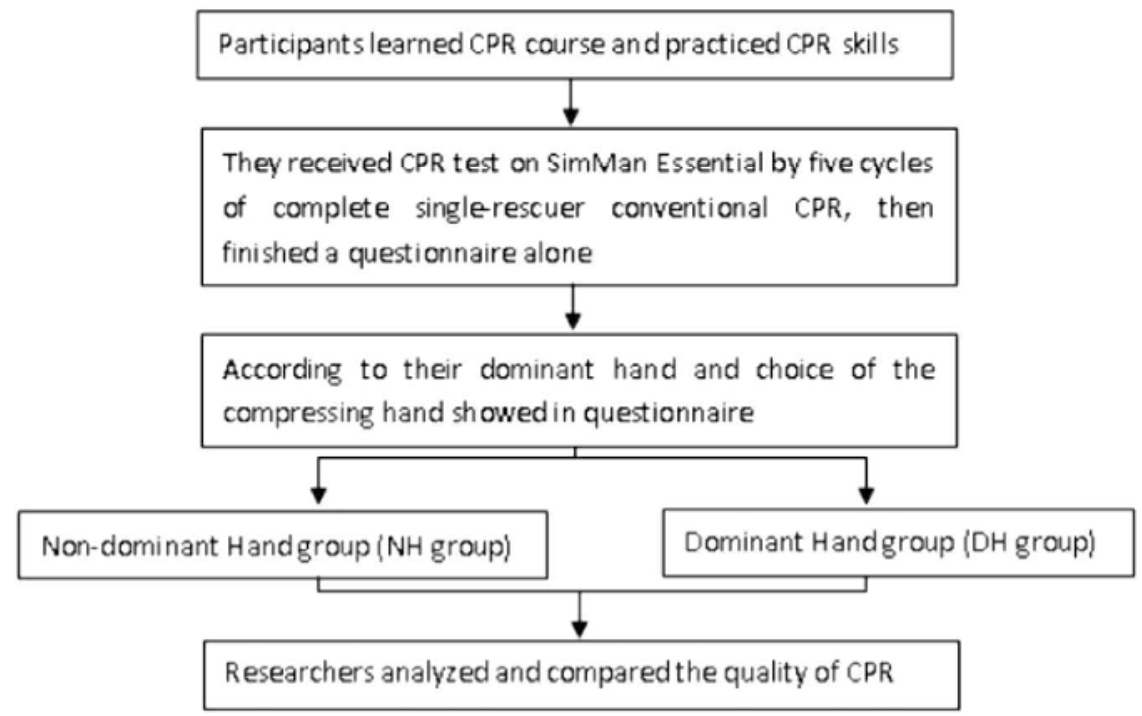

Figure 1. Schema of the study. CPR = cardiopulmonary resuscitation.

SPSS Inc., Chicago, IL). All $p$ values were two-tailed, and $p$ values $<0.05$ were considered significant.

\section{RESULTS}

General Characteristics and Evaluations by the BLS Instructor

There were 228 students involved in the CPR course and test. There was no one changing hand in contact with the sternum during CPR. After the final collection of all of the data, three students were excluded because they

Table 1. General Characteristics of the NH and DH Groups

\begin{tabular}{lcc}
\hline & NH Group $(\mathrm{n}=155)$ & DH Group $(\mathrm{n}=65)$ \\
\hline Age, years & & \\
$18-24$ & $110(71 \%)$ & $48(74 \%)$ \\
$25-31$ & $44(28 \%)$ & $17(26 \%)$ \\
$>31$ & $1(1 \%)$ & $0(0 \%)$ \\
Gender & & \\
Male & $59(38 \%)$ & $30(46 \%)$ \\
Female & $96(62 \%)$ & $35(54 \%)$ \\
Height $(\mathrm{cm})$ & & \\
$150-159$ & $38(25 \%)$ & $12(19 \%)$ \\
$160-169$ & $90(58 \%)$ & $27(42 \%)$ \\
$170-179$ & $23(15 \%)$ & $4(34 \%)$ \\
$180-189$ & $4(2 \%)$ & $14(22 \%)$ \\
Weight $(\mathrm{kg})$ & $31(20 \%)$ & $22(34 \%)$ \\
$40-49$ & $67(43 \%)$ & $17(26 \%)$ \\
$50-59$ & $39(25 \%)$ & $10(15 \%)$ \\
$60-69$ & $17(11 \%)$ & $2(3 \%)$ \\
$70-79$ & $1(1 \%)$ & \\
$80-89$ & &
\end{tabular}

$\mathrm{NH}=$ nondominant hand; $\mathrm{DH}=$ dominant hand.

The data are presented as numbers (percentages). Note: some of the items were missing: two cases in the $\mathrm{NH}$ group and three cases in the DH group did not provide all general characteristics. completed only two cycles of CPR. One of these students was pregnant, one experienced carpitis, and one refused to perform all five cycles of CPR. Thus, we included totals of 157 participants in the NH group and 68 participants in the DH group. Some data were missing: the general characteristics of two cases in the $\mathrm{NH}$ group and three cases in the DH group were missing, no answer to question 3 was provided by one case in the $\mathrm{NH}$ group, and no answer was provided for question 8 by one case in the DH group.

There were no significant between-group differences in the main characteristics, with the exception of height (Table 1). There were also no significant differences in the manual scores (Table 2).

Table 2. CPR Scores from the BLS Instructor and Total Chest Compression Qualities from the SimMan Essential of the $\mathrm{NH}$ and $\mathrm{DH}$ Groups

\begin{tabular}{|c|c|c|c|c|}
\hline & $\begin{array}{l}\text { NH Group } \\
(\mathrm{n}=157)\end{array}$ & $\begin{array}{l}\text { DH Group } \\
(\mathrm{n}=68)\end{array}$ & $\begin{array}{l}p \text { Value } \\
\text { (Means) }\end{array}$ & $\begin{array}{c}p \text { Value } \\
\text { (Variance) }\end{array}$ \\
\hline Scores & $88 \pm 4$ & $88 \pm 3$ & 0.98 & 0.31 \\
\hline $\begin{array}{l}\text { Rate (cpm) } \\
\quad<100 \\
\geq 100\end{array}$ & $\begin{array}{c}61(8 \%) \\
724(92 \%)\end{array}$ & $\begin{array}{c}10(3 \%) \\
330(97 \%)\end{array}$ & 0.002 & NA \\
\hline Depth (mm) & & & 0.001 & NA \\
\hline $\begin{array}{l}<50 \\
\geq 50\end{array}$ & $\begin{array}{l}630(80 \%) \\
155(20 \%)\end{array}$ & $\begin{array}{r}241(71 \%) \\
99(29 \%)\end{array}$ & & \\
\hline Recoil (\%) & & & 0.02 & NA \\
\hline $\begin{array}{l}<100 \\
=100\end{array}$ & $118(15 \%)$ & $\begin{array}{r}70(21 \%) \\
270(79 \%)\end{array}$ & & \\
\hline
\end{tabular}

CPR = cardiopulmonary resuscitation; BLS = basic life support; $\mathrm{NH}$ group = nondominant hand group; $\mathrm{DH}$ group = dominant hand group; $\mathrm{cpm}=$ compressions per minute; $\mathrm{NA}=$ unavailable or not performed.

The data are presented as the means \pm the SDs or as numbers (percentages). 


\section{Chest Compression Rate}

The chest compression rates were $131 \pm 21 \mathrm{cpm}$ for the $\mathrm{NH}$ group and $133 \pm 18 \mathrm{cpm}$ for the DH group ( $p=$ $0.18)$. There were no differences between the cycles across the two groups or across the five cycles within the NH or DH group. We also analyzed the frequency distributions of the chest compression rates of each group. The frequency of chest compressions that were "above $100 \mathrm{cpm}$ " was greater in the DH group than in the $\mathrm{NH}$ group ( $p=0.002$, Table 2). Moreover, the distributions were significantly different between the two groups ( $p$ $<0.0001$; Figure 2). The highest proportion of the distribution of the NH group was "130-139" cpm, but in the DH group, this value was "140-149" cpm.

\section{Chest Compression Depth}

The chest compression depths were $43 \pm 8 \mathrm{~mm}$ in the $\mathrm{NH}$ group and $44 \pm 8 \mathrm{~mm}$ in the $\mathrm{DH}$ group ( $p=$ 0.001). There were no differences between any cycles across the two groups. In the NH group, the depth in cycle 5 (41 $\pm 8 \mathrm{~mm})$ was significantly less than those of cycle $1(44 \pm 7 \mathrm{~mm}, p=0.002)$, cycle $2(43 \pm 7 \mathrm{~mm}, p=$ $0.006)$, and cycle 3 (43 $\pm 8 \mathrm{~mm}, p=0.04)$. However, in the DH group, there were no significant differences between the cycles (Table 3). The frequency of depths $>50$ $\mathrm{mm}$ in the DH group was higher than that of the NH group $(p=0.001)$, as shown in Table 2 .

\section{Chest Recoil}

The chest recoils were $98 \% \pm 8 \%$ in the $\mathrm{NH}$ group and $97 \% \pm 10 \%$ in the $\mathrm{DH}$ group $(p=0.13)$. There were no differences between any cycle between the two groups or

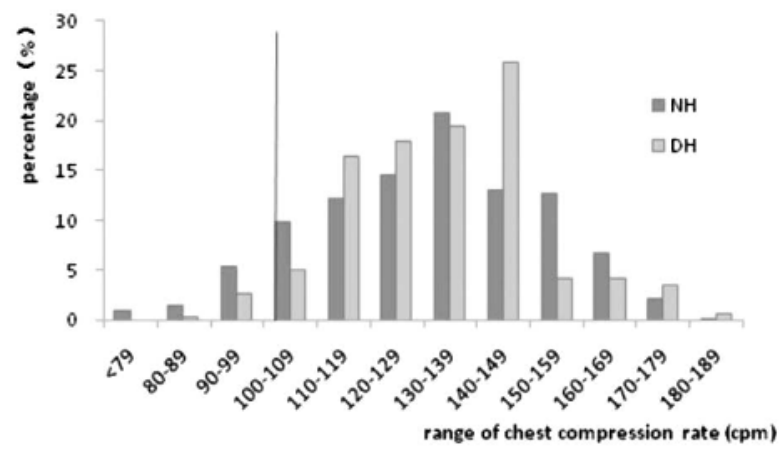

Figure 2. Frequency distributions of the chest compression rates of the NH and DH groups. There were significant differences in the distributions between the two groups $(p<$ 0.0001 ). The line indicates the chest compression rate of $100 \mathrm{cpm}$, which is the minimum recommended chest compression rate according to the American Heart Association 2010 CPR guidelines. $\mathrm{NH}=$ nondominant hand; $\mathrm{DH}=$ dominant hand; $\mathrm{cpm}=$ compressions per minute.
Table 3. Chest Compression Depths and Chest Recoils of the NH and DH Groups

\begin{tabular}{ccccc}
\hline & $\begin{array}{c}\text { NH Group } \\
(\mathrm{n}=157)\end{array}$ & $\begin{array}{c}\text { DH Group } \\
(\mathrm{n}=68)\end{array}$ & $\begin{array}{c}p \text { Value } \\
\text { (Means) }\end{array}$ & $\begin{array}{c}p \text { Value } \\
\text { (Variance) }\end{array}$ \\
\hline Depth (mm) & $43 \pm 8$ & $44 \pm 8$ & 0.001 & 0.37 \\
Cycle 1 & $44 \pm 7^{\star}$ & $45 \pm 8$ & & \\
Cycle 2 & $43 \pm 7^{\star}$ & $45 \pm 7$ & & \\
Cycle 3 & $43 \pm 8^{\star}$ & $44 \pm 8$ & & \\
Cycle 4 & $42 \pm 8$ & $44 \pm 8$ & & \\
Cycle 5 & $41 \pm 8$ & $43 \pm 8$ & & \\
Recoil (\%) & $98 \pm 8$ & $97 \pm 10$ & 0.17 & \\
Cycle 1 & $99 \pm 5$ & $97 \pm 7$ & & \\
Cycle 2 & $98 \pm 8$ & $97 \pm 11$ & & \\
Cycle 3 & $98 \pm 9$ & $97 \pm 11$ & & \\
Cycle 4 & $98 \pm 9$ & $97 \pm 11$ & & \\
Cycle 5 & $98 \pm 8$ & $98 \pm 9$ & & \\
\hline
\end{tabular}

$\mathrm{NH}=$ nondominant hand; $\mathrm{DH}=$ dominant hand.

The data are presented as the means \pm the SDs.

${ }^{\star} p<0.05$ vs. cycle 5 in the $\mathrm{NH}$ group.

across the five cycles within the NH group or DH group (Table 3). More of the chest recoils were full in the $\mathrm{NH}$ group $(p=0.02$, Table 2$)$.

\section{CPR Training Questionnaire}

Across all of the questionnaires, one participant in the $\mathrm{NH}$ group did not complete question 3, and one participant in the DH group responded to question 8 with "no fatigue" rather than choosing a single answer.

Within each group, most of the students were righthanded (98\% in the $\mathrm{NH}$ group and $65 \%$ in the $\mathrm{DH}$ ). Most students in the $\mathrm{NH}$ group believed that the use of the nondominant hand would optimize CPR quality (78\% with the left hand and $9 \%$ with the right hand). In the DH group, the students primarily believed that the use of the dominant would be better $(26 \%$ with the left hand and $46 \%$ with the right hand). In the $\mathrm{NH}$ and $\mathrm{DH}$ groups, $13 \%$ and $28 \%$ of the students, respectively, believed that the identity of the compression hand did not influence CPR quality.

Regarding chest compression rates, $66 \%$ of the students in the $\mathrm{NH}$ group and $69 \%$ in the DH group believed that they had achieved compression rates above $100 \mathrm{cpm}$. Regarding chest compression depth, $62 \%$ of the students in the $\mathrm{NH}$ group and $72 \%$ in the $\mathrm{DH}$ group believed that they had achieved chest compression accuracies above $76 \%$. Regarding chest recoil, $32 \%$ of the students in the $\mathrm{NH}$ group and $32 \%$ in the DH group believed that they had achieved complete chest recoil. Regarding chest compression location, $78 \%$ of students in the NH group and $79 \%$ in the DH group believed that they had achieved accuracies above $76 \%$. Regarding fatigue, the students felt fatigue at different time points $(43 \%$ in the $\mathrm{NH}$ and $40 \%$ in the DH groups after the fourth cycle, and $39 \%$ in the $\mathrm{NH}$ and $45 \%$ in the $\mathrm{DH}$ groups after the fifth cycle). 
Ninety-nine percent of the students in each of the $\mathrm{NH}$ and DH groups believed that they had nearly or completely mastered CPR during their training.

\section{DISCUSSION}

Chest compression is an especially important component of CPR. Chest compression provides sufficient blood flow when sudden cardiac arrest occurs. To optimize the quality of CPR, the AHA 2010 CPR guidelines changed the points of emphasis regarding chest compression. The newer guidelines stipulate that the adult chest should be compressed at a rate of at least $100 \mathrm{cpm}$ and that the compression depth should be at least $50 \mathrm{~mm}$ to allow for complete chest recoil and to minimize the frequency and duration of interruptions in compressions (3). Several factors can affect the quality of chest compression, such as the position of victim, the position of rescuer, hand position, the depth and rate of compression, recoil, and the duty cycle (6). We found having the dominant hand in contact with the sternum during compression resulted in the following: 1) trends toward greater chest compression rates and deeper chest compressions; 2) a trend toward incomplete chest recoil; and 3) the delaying of fatigue compared to the use of the nondominant hand.

Kundra et al. showed that external chest compression is performed with fewer errors when the dominant hand of the rescuer is placed in contact with the sternum (4). This result was mainly due to the significant alteration in the magnitude of the force that is applied to the manikin (4). Nikandish et al. also found a trend toward an increasing incidence of correct chest compressions when the dominant hand is positioned to be the lower one (5). Our study used the SimMan Essential, which is a realistic full-body adult wireless patient simulator that offers comprehensive clinical functionality for the practice of airway, breathing, cardiac, and circulation skills. Some researchers have reported clinical improvements or equal performances when advanced realistic manikins are used, rather than less technologically advanced manikins, in clinical education (7-10). The AHA 2010 CPR guidelines also state that the evidence is insufficient to advocate for or against the routine use of more realistic manikins (11). However, the advantage of the manikin used here is its clear, objective, and easyto-read demonstration of CPR performance, which provides a more convenient method for monitoring, analysis, and feedback. We found that the evaluation scores that were based on traditional evaluation methods and given by the BLS instructor were not different between the two groups. However, the analyses of each detailed performance from the SimMan Essential revealed important differences.
We wanted to obtain the primary parameters of chest compression quality from the SimMan Essential. Thus, we did not analyze the hand location during the compressions, which are shown by the computer only as hands that emerge in or outside of a "correct location circle" and not as a number or percentage. Regarding chest compression rates, both groups achieved the recommended rate (at least $100 \mathrm{cpm}$ ), and a higher proportion among the DH group achieved this rate. Comparisons revealed that a higher proportion of the DH group compressed more rapidly (140-149 cpm) (Figure 2). In both groups, the chest compression depths were less than the recommended depth (at least $50 \mathrm{~mm}$ ), but a lower proportion of the DH group failed to meet this recommended depth. Although they were not deep enough, the compressions of the DH group were still $1 \mathrm{~mm}$ deeper than those of the NH group. Regarding chest recoil, neither group achieved complete recoil, but the proportion of participants that achieved $100 \%$ recoil in the DH group was lower than that of the $\mathrm{NH}$ group.

Hand dominance refers to the preference of one hand for performing fine and gross motor skills. Humans may have instinctive tendencies to perform tasks with the dominant hand to ensure that the required force, direction, and precision are applied. Our study was not designed to interfere with the choices of the participants. Thus, we did not ask the participants to perform CPR twice using each hand as the compression hand, as was done in the studies by Kundra et al. and Nikandish et al. $(4,5)$. Therefore, we believe that we observed instinctual CPR performances, which may help us to identify the primary differences between the use of the dominant and nondominant hands. Similar to the results reported by Kundra et al., we observed superior chest compression performances in terms of compression rate and depth when the dominant hand was used (4). However, the proportion of full chest recoils was lower in the DH group. We believe that the faster and harder chest compressions might not have allowed for sufficiently complete chest relaxation. The depths of both groups were $<50 \mathrm{~mm}$, and they performed CPR on the manikin's right side. You et al. reported that a significant decrease in mean compression depth is observed when rescuers perform on the right side of the manikin with the left hand in contact with the sternum, regardless of hand dominance (12). Geddes et al. found that trained CPR rescuers exert more force than do laypersons, and thus perform more effective CPR (13). We presume that the students involved in our study could improve their chest compression depths via further training.

We compared the participants' questionnaire results to the actual performances on the manikin. The assessments were approximately the same in terms of the accuracy of the chest compression rates. The students strongly 
overestimated the accuracies of their chest compression depths, because only $20 \%$ of the students in the NH group and $29 \%$ in the DH group produced sufficient chest compression depths. However, the students underestimated their performances in terms of chest recoil. In the $\mathrm{NH}$ and DH groups, $85 \%$ and $79 \%$ of the students, respectively, achieved complete chest recoil, and these percentages were higher than those reported in the students' own assessments (32\% in the $\mathrm{NH}$ and $32 \%$ in the $\mathrm{DH}$ groups). These results again verify the benefits of the use of advanced manikin examination analyses and feedback in CPR training. Moreover, these results may help instructors adjust future training courses.

Significant fatigue and shallow compressions are common after $1 \mathrm{~min}$ of CPR, although rescuers may not recognize that fatigue is present for more than $5 \mathrm{~min}$ (14). Our participants felt fatigue mostly from the fourth or fifth cycle on. The manikin data revealed that the participants in the $\mathrm{NH}$ group made significantly shallower compressions beginning in the fifth cycle, which is an obvious indicator of fatigue. However, no such difference was observed in the DH group. It seems intuitive that the dominant hand would be capable of sustaining the proper force and control for a longer period of time than the nondominant hand.

With the exception of height, there were no differences between the two groups in terms of general characteristics. Ødegaard et al. used a manikin to show that compression depth does not depend on rescuer gender, height, or weight (15). Nikandish et al. and Sandroni et al. showed that the rescuer's weight and height do not influence compression quality $(5,16)$. We also analyzed the correlations between chest compression parameters (i.e., rate, depth, and recoil) and general characteristics (i.e., gender, height, and weight). We found that chest compression quality exhibited nearly no correlation with gender, height, or weight (data not shown).

\section{Limitations}

First, although we used an advanced realistic manikin, we did not examine real clinical resuscitations of patients. The feeling of the compressions, and the controls and motivations vary to some extent between humans and manikins, and this variation may have influenced the students' CPR performances. Secondly, the subjects of the study were students. Although our previous study showed that students are able to perform CPR as well as the medical staffs of emergency departments, we believe that it remains necessary to observe CPR performances in terms of the position of the dominant hand among different populations, such as skillful health care providers and the public (17). Third, we did not obtain objective results regarding chest compression location, which is also an important component of chest compression.

\section{CONCLUSIONS}

Our study showed that the use of the dominant hand in the compression position during CPR can produce higherquality CPR via greater chest compression rates, deeper chest compressions, and delayed fatigue.

Acknowledgments - We would like to thank all of the staff of the American Heart Association Emergency Cardiovascular Care Training Center (Zhongnan Hospital of Wuhan University) and the Emergency Diagnostic \& Therapeutic Center of Central China. Their supports made this study possible.

\section{REFERENCES}

1. Eisenberg MS, Mengert TJ. Cardiac resuscitation. N Engl J Med 2001;344:1304-13.

2. Becker LB. The epidemiology of sudden death. In: Paradis NA, Halperin HR, Nowak RM, eds. Cardiac arrest: the science and practice of resuscitation medicine. Baltimore, MD: Williams \& Wilkins; 1996:28-47.

3. Berg RA, Hemphill R, Abella BS, et al. Part 5: adult basic life support: 2010 American Heart Association Guidelines for Cardiopulmonary Resuscitation and Emergency Cardiovascular Care. Circulation 2010;122(18 Suppl 3):S685-705

4. Kundra P, Dey S, Ravishankar M. Role of dominant hand position during external cardiac compression. Br J Anaesth 2000; $84: 491-3$

5. Nikandish R, Shahbazi S, Golabi S, Beygi N. Role of dominant versus non-dominant hand position during uninterrupted chest compression CPR by novice rescuers: a randomized double-blind crossover study. Resuscitation 2008;76:256-60.

6. Wik L, Kramer-Johansen J, Myklebust H, et al. Quality of cardiopulmonary resuscitation during out-of-hospital cardiac arrest. JAMA 2005;293:299-304.

7. Wayne DB, Didwania A, Feinglass J, Fudala MJ, Barsuk JH, McGaghie WC. Simulation-based education improves quality of care during cardiac arrest team responses at an academic teaching hospital: a case control study. Chest 2008;133:56-61.

8. Owen H, Mugford B, Follows V, Plummer JL. Comparison of three simulation-based training methods for management of medical emergencies. Resuscitation 2006;71:204-11.

9. Schwartz LR, Fernandez R, Kouyoumjian SR, Jones KA, Compton S. A randomized comparison trial of case-based learning versus human patient simulation in medical student education. Acad Emerg Med 2007;14:130-7.

10. Hoadley TA. Learning advanced cardiac life support: a comparison study of the effects of low- and high-fidelity simulation. Nurs Educ Perspect 2009;30:91-5.

11. Bhanji F, Mancini ME, Sinz E, et al. Part 16: education, implementation, and teams: 2010 American Heart Association Guidelines for Cardiopulmonary Resuscitation and Emergency Cardiovascular Care. Circulation 2010;122:S920-33.

12. You JS, Kim H, Park JS, et al. Relative effectiveness of dominant versus non-dominant hand position for rescuer's side of approach during chest compressions between right-handed and left-handed novice rescuers. Emerg Med J 2013; http://dx.doi.org/10.1136/ emermed-2013-202515. [Epub ahead of print].

13. Geddes LA, Boland MK, Taleyarkhan PR, Vitter J. Chest compression force of trained and untrained CPR rescuers. Cardiovasc Eng $2007 ; 7: 47-50$ 
14. Manders S, Geijsel FE. Alternating providers during continuous chest compressions for cardiac arrest: every minute or every two minutes? Resuscitation 2009;80:1015-8.

15. Ødegaard S, Kramer J, Bromley A. Chest compressions by ambulance personnel on chests with variable stiffness: abilities and attitudes. Resuscitation 2007;74:127-34.
16. Sandroni C, Bocci MG, Damiani F, Proietti R, Speranza D. Can the body size affect students' performance during CPR training. Resuscitation 1997;34:191.

17. Zhou XL, Duan XW, Zhao Y, et al. Medical students do not adversely affect the quality of cardiopulmonary resuscitation for ED patients. Am J Emerg Med 2014;32:306-10. 


\section{APPENDIX:}

\section{CARDIOPULMONARY RESUSCITATION (CPR) TRAINING QUESTIONNAIRE}

Hello, dear student!

Thank you for attending the CPR training course. This survey is to help us learn more about your CPR studies and is not a test. Please choose one answer for each question with a “ $\checkmark$.

Thank you for your honest replies.

\begin{tabular}{|l|l|l|l|l|}
\hline Name & Age & Sex & Height cm & Weight Kg \\
\hline
\end{tabular}

1、Which statement best describes you?
A. Right-hand dominant
B. Left-hand dominant

2、Which statement best describes your CPR performance just now?

A. Right hand in contact with the chest, left hand overlapping the right hand

B. Left hand in contact with the chest, right hand overlapping the right hand

3. The placement of which hand in contact with the chest would optimize the quality of CPR?
A. Left hand
B. Right hand
C. No difference between the left and right

4. What is your opinion about the accuracy of your chest compression location during the CPR you performed just now?
A. $<25 \%$
B. $26-50 \%$
C. $51-75 \%$
D. $\geqslant 76 \%$

5. What is your opinion about the accuracy of the chest compression depth of the CPR you performed just now?
A. $<25 \%$
B. $26-50 \%$
C. $51-75 \%$
D. $\geqslant 76 \%$

6、What is your opinion about the chest compression rate of the CPR you performed just now?
A. $<80 \mathrm{cpm}$
B. $80-90 \mathrm{cpm}$
C. $90-100 \mathrm{cpm}$
D. $>100 \mathrm{cpm}$

7、What is your opinion about the chest recoil of the CPR you performed just now?
A. No recoil
B. Less recoil
C. Most recoil
D. Complete recoil

8. In which cycle did you feel fatigue?
A. 1
B. 2
C. 3
D. 4
E. 5

9. Please give yourself a score regarding your learning of CPR.

A. 1 (no mastery) B. 2 (unsure mastery) C. 3 (nearly mastery) D. 4 (complete mastery) This is the end of the questionnaire. Thank you for your attendance and support! 


\section{ARTICLE SUMMARY}

1. Why is this topic important?

According to the 2010 CPR guideline, external chest compression becomes the core of cardiopulmonary resuscitation (CPR). Although many factors can affect the quality of chest compression, hand choice to be the lower compressing one has not been clearly identified.

\section{What does this study attempt to show?}

We designed to explore the dominant hand position influences according to the new CPR guidelines, through an advanced manikin system.

\section{What are the key findings?}

We found that the dominant hand position in contact with the sternum during compression resulted in the following: 1) trends toward greater chest compression rates and deeper chest compressions; 2) a trend toward incomplete chest recoil; and 3) the delaying of fatigue compared to the use of the nondominant hand. 
Reply to Letter to the Editor

\section{Reply to Letter: Video recording and feedback of resuscitation}

Sir,

We are grateful for the opportunity to reply to the helpful comments about our study made by Dr Ma and colleagues.

In our series of cardiac arrests, a total of 16 cases which were caused by haemorrhage (trauma, extrauterine pregnancy, cerebral haemorrhage). The treatment protocols for traumatic arrests include not just the ABCs, but also specific surgical interventions and coordinated team work. However, the aim of our study was to describe how recording of CPR performed in our emergency department (including all the adult cases) with real-time video and regular feedback learning may improve CPR - our emphasis was the quality of CPR. We still contend that we can improve the outcome of resuscitation from traumatic cardiac arrest by using video feedback.

The methods used to review the video recordings were described in our paper. The reviewers were trained to watch videos in the same mode, to record the time period, number of chest compressions and activities needed in a prospectively designed template. Both reviewers were blind to the date, time and other information in the video. If an assessment of one recording was different between two observers, a third expert physician was consulted to give the final decision. The lead investigator devised the indicators used in our study, which mainly reflected CPR quality. The feedback of the video recording to our resuscitation teams was undertaken every week. The attendants watched the video recording and discussed their performance according to our main indicators of CPR quality: (1) the initiation time of CPR; (2) instantaneous rates of chest compression; (3) hands-off time; and (4) intubation time.

We recorded the duration of each CPR session and calculated for other indicators such as average chest compression count in every minute (count/manual compression resuscitation Time $\left(T_{1}\right)$ ). We analyzed the $T_{1}$ for three groups: $13.17 \pm 10.10(\mathrm{~min})$ (group 1), $19.67 \pm 11.01$ ( $\mathrm{min}$ ) (group 2) and $14.49 \pm 10.61$ (min) (group 3), which showed no significant difference among groups $(F=1.582$, $P=0.218$ ).
The hands-off time definition varies with different studies: Abella et al. recommended $1.5 \mathrm{~s}$, ${ }^{1}$ Wang et al. recommended periods longer than $2 \mathrm{~s},{ }^{2}$ and another paper by Abella et al. recommended periods longer than $4 \mathrm{~s} .{ }^{3}$ In our study, we did not want unnecessary hands-off time, so we defined it as longer than $1 \mathrm{~s}$.

\section{Conflict of interest statement}

None.

\section{Acknowledgment}

We would like to thank Peng Yaqiong, lecturer of Foreign language department, Shanghai Institute of Electric Power, for the language editing to this paper.

\section{References}

1. Abella BS, Alvarado JP, Myklebust H, et al. Quality of cardiopulmonary resuscitation during in-hospital cardiac arrest. JAMA 2005;293:305-10.

2. Wang HC, Chiang WC, Chen SY, et al. Video-recording and time-motion analyses of manual versus mechanical cardiopulmonary resuscitation during ambulance transport. Resuscitation 2007;74:453-60.

3. Abella BS, Sandbo N, Vassilator P, et al. Chest compression rates during cardiopulmonary resuscitation are suboptimal. Circulation 2005;111:428-34.

\section{Jiang Cheng} Zhao Yan*

Zhongnan Hospital of Wuhan University, Emergency Department, 169 Donghu Road, Wuchang District, Wuhan City, Hubei Province, Zip Code: 430071, China

*Corresponding author. Tel.: +86 13995577963. E-mail addresses: sharroncandy@yahoo.com.cn (C. Jiang), yz2711@gmail.com (Y. Zhao)

4 January 2012 


\section{RÉSUMÉ}

Le système nerveux sympathique (SNS) a été identifié à être progressivement activé dans de nombreuses maladies cardio-vasculaires, du processus chroniques différents, y compris l'hypertension et de cardiomyopathie, à l'insuffisance cardiaque congestive ${ }^{[82,156,412-414]}$. L'hyperactivité du système nerveux sympathique peut modifier le nombre, la fonction et la voie de signalisation en aval des récepteurs $\beta$-adrénergiques. Bien qu'il n'yait pas de mécanisme unifié pour interpréter ces découvertes divergentes, des anomalies de calcium ont été reconnu comme une cause fondamental de la fonction systolique défectueuse du ventricule gauche ${ }^{[82]}$. Le niveau de calcium intracellulaire est directement impliqué dans l'interaction entre l'actine et la myosine, qui controle la contractilité du muscle. Les stimulations des récepteurs $\beta$-adrénergiques peuvent induire des réponses inotropes / lusitropes positifs par la production d'adénosine monophosphate cyclique (AMPc) et l'activation de la protéine kinase A (PKA). Le calcium de type transitoire est augmenté suite à la phosphorylation des protéines ciblées (cannaux calciques, récepteurs de la ryanodine, SERCA2a et troponine). Dans les conditions physiologique, la dysfonctionnement des récepteurs $\beta$-adrénergiques peut être un mécanisme commun de la réduction de la fonction cardiaque. Dans ce contexte, nous avons effectué des expériences au sujet de la voie de stimulation $\beta$-récepteurs adrénergiques en deux états physiopathologiques différents, cardiomyopathie du syndrome métabolique et de personnes âgées.

La première expérience est menée au sein de rats sénescents. Au coeur sénescent, un dysfonctionnement diastolique et une réponse réduite à la stimulation $\beta$-adrénergiques ont été identifiés, qui sont associés à une sous-expression des récepteurs $\beta_{1}$ - et $\beta_{2}$-adrénergiques, ainsi qu'à une sur-expression des récepteurs $\beta_{3}$-adrénergiques. ${ }^{[73,415,416]}$ Ces changements permettent de réduire la production d'AMPc ou facilitent l'hydrolyse de l'AMPc. Ainsi, un mécanisme complémentaire de la régulation de l'AMPc a été impliqué dans le cœur. La protéine de multirésistance 4 (MRP4) joue un rôle important dans la régulation de l'AMPc intracellulaire et les réponses cardiaques stimulées par les récepteurs $\beta$-adrénergiques. Mais le rôle de MRP4 au coeur sénescent n'a jamais été étudié. Dans ce fait, nous avons mené de l'expérience pour étudier l'expression de MRP4 et son influence sur le dysfonctionnement $\beta$ adrénergique dans le cœur sénescent. L'expression de MRP4 a été quantifiée dans ventriculaires gauches par Western Blotting. Les réponses des récepteurs $\beta$-adrénergiques à l'isoprotérénol ont été étudiés in vivo (échocardiographie de stress) et in vitro (raccourcissement des sarcomères et de calcium de type transitoire dans les cardiomyocytes isolés par Ionoptix ${ }^{\circledR}$ ) chez les rats jeunes (âgés de 3 mois) et les rats sénescents (âgés de 24 mois) prétraités ou non avec MK571, un inhibiteur spécifique de MRP4. En conséquence, nous avons confirmé que la sur-expression de MRP4 contribue à la diminution de la réponse inotrope positive du coeur sénescent à la stimulation de la $\beta$-adrénorécepteurs. La deuxième experience est menée au sein des rats du syndrome métabolique. Bien que le syndrome métabolique est associé à une augmentation de l'activité sympathique qui stimule chroniquement les récepteurs $\beta$-adrénergiques, la voie de signalisation de ces récepteurs impliqués dans cette situation a été très peu étudiée. En utilisant le model des rats Zucker témoins, obèses et obèses diabétiques (de type 2), nous avons étudié la voie de signalisation de ces récepteurs $\beta$-adrénergique. Nous avons comparé la voie de signalisation des $\beta$ récepteurs adrénergiques dans les rats Zucker témoins, Zucker obèses, et Zucker obèses diabétiques, qui sont les modèles de rats fiables du syndrome métabolique. Les effets de la stimulation des récepteurs $\beta$-adrénergiques ont été évalué in vivo avec l'échocardiographie transthoracique et in vitro dans les muscles papillaires ventriculaires gauches des rats Zucker témoins, obèses et obèse diabétiques. L'expression des récepteurs $\beta_{1^{-}}, \beta_{2^{-}}$, et $\beta_{3^{-}}$-adrénergiques et de la proteine 4 associée aux resistances multidrogues dans les muscles ventriculaires 
gauches a été quantifiée par Western Blotting. Les concentrations du triglycéride, du cholestérol, de la leptine, de l'adiponectine, et du peptide-C dans le plasma sanguin ont été mesurées avec la méthod enzymatique, ou la method immuno-enzymatique quantitative ELISA. Les données sont présentées en moyenne \pm SD. L'hyperlipidémie et des concentrations élevées de la leptine et du peptide-C ont été observés dans des souches obèses diabétiques et obèses, alors que l'hyperglycémie n'est présentée que dans la souche diabétique. Aucune différence significative a été observée entre les souches in vivo en échocardiographie. In vitro, l'effet inotrope positif de l'isoprotérénol est légèrement réduite chez les rats obèses $(183 \% \pm 11 \%$ de la valeur de base, $P=0,003 ; \mathrm{n}=7)$ et nettement réduite chez les rats obèses diabétiques $(137 \% \pm 18 \%$ de la valeur de base, $P<0,001 ; \mathrm{n}=10)$ par rapport aux rats témoins $(210 \% \pm 17 \%$ de la valeur de base; $n=9)$. L'expression du récepteur $\beta_{1}$-adrénergique est diminué chez les rats obèses $(-41 \%, P=0,02)$ et les rats diabétiques $(-54 \%, P=0,003)$ par rapport aux rats témoins, et une diminution similaire a été observée en l'expression du récepteur $\beta_{2}$-adrénergique. Alors que l'expression des récepteurs $\beta_{3}$-adrénergiques et la protein 4 associée aux resistances multidrogues reste inchangée. L'effet lusitrope de l'isoprotérénol n'est pas modifié entre les souches. L'effet inotrope positif de la forskoline est légèrement diminué chez les rats obèses $(148 \% \pm 25 \%$ de la valeur de base, $P=0,005)$ et nettement diminuée chez les rats obèses diabétiques $(118 \% \pm 12 \%$ de la valeur de base , $P$ $<0,001)$ par rapport aux rats témoins $(181 \% \pm 23 \%$ de la valeur de base). Lors de la stimulation de la 3', 5'-adénosine monophosphate cyclique par le dibutyryl cAMP, l'effet inotrope positif chez les rats témoins $(185 \% \pm 19 \%$ de la valeur de base $)$ est significativement plus élevée que celui chez les rats obèses et chez les rats obèses diabétiques $(158 \% \pm 36 \%$ et $155 \% \pm 27 \%$ de la valeur de base; $P=0,043$ et $P=0,031$, respectivement). Outre une dimimution de l'expression du récepteur $\beta_{1}$ - et $\beta_{2}$-adrénergique, la stimulation directe de l'adénylate cyclase par la forskoline et l'administration de la 3', 5'-adénosine monophosphate cyclique suggère une déficience subtiles s'est produit également au-dessus et au-dessous du niveau de voie les $\beta$-adrénergique chez les rats obèses et diabétiques. En somme, l'effet inotrope positif de la stimulation des récepteurs $\beta$-adrénergiques est légèrement diminué dans les rats Zucker obèses et diminué plus nettement dans les rats Zucker obèses diabétiques. Ces diminuations sont principalement liées à une sous-expression du récepteurs $\beta_{1}$ - et $\beta_{2^{-}}$ adrénergiques.

Mots clés récepteurs $\beta$-adrénergiques; muscle cardiaque; catécholamines; l'obésité; le diabète 


\section{ABSTRACT}

The sympathetic nervous system (SNS) has been identified to be progressively activated in many cardiovascular diseases, from different chronic process including hypertension and cardiomyopathy, to congestive heart failure ${ }^{[82,156,412-414]}$. The hyperactivity of sympathetic nervous system may change the number, function and downstream mechanisms of $\beta$ adrenoceptors. Although there is no unified mechanism to interpret those divergent findings, calcium abnormalities has been recognized to be fundamental in the defective systolic function in left ventricle ${ }^{[82]}$. The intracellular calcium level is directly involved in the interaction of actin and myosin, thus reflects the contractility of muscle. $\beta$-adrenergic stimulation can induce the positive inotropic / lusitropic responses via the production of cyclic adenosine monophosphate (cAMP) and the activation of protein kinase A (PKA). Thus calcium transient is increased after phosphorylation of serials targeted proteins (calcium channel, ryanodine receptor, SERCA2a, and troponin). Under the pathophysiological condition, $\beta$-adrenergic dysfunction may be a common mechanism of decreased cardiac function. So, we performed experiments about $\beta$-adrenoceptor stimulation pathway in two different pathophysiological status, cardiomyopathy of the elderly and metabolic syndrome.

The first experiment is conducted within senescent rat. In the senescent heart, diastolic dysfunction and reduced response to $\beta$-adrenergic stimulation have been identified, which are associated with the down-regulation of $\beta_{1}$ - and $\beta_{2}$-adrenoceptors, along with the up-regulation of $\beta_{3}$-adrenoceptor. ${ }^{[7,415,416]}$ These changes either reduce the cAMP production or facilitate the hydrolysis of cAMP. Meanwhile, a complementary mechanism of the regulation of cAMP has been involved in heart. The multidrug resistance protein 4 (MRP4) plays an important role in the regulation of intracellular cAMP and $\beta$-adrenergic stimulated cardiac responses. But the role of MRP4 in the senescent heart has never been studied. Thus, we conducted the experiment to study the MRP4 expression and its influence on $\beta$-adrenergic dysfunction in the senescent rat heart. MRP4 was quantified in left ventricular homogenates by Western blotting. The $\beta$-adrenergic responses to isoproterenol were investigated in viv o (stress echocardiography) and in $v$ itro (sarcomere shortening and calcium transient of isolated cardiomyocyte by Ionoptix ${ }^{\circledR}$ ) in young (3-month age) and senescent (24-month age) rats pretreated or not with MK571, a specific MRP4 inhibitor. As a result, we confirmed that the MRP4 overexpression contributes to the decrease of positive inotropic response to $\beta$ adrenoceptor stimulation in the senescent heart. The second experiment is conducted within metabolic syndrome rats. Although metabolic syndrome is associated with increased sympathetic activity that chronically stimulates $\beta$-adrenoceptors, the $\beta$-adrenoceptor signaling pathway has been poorly studied in this situation. We studied the $\beta$-adrenoceptor signaling pathway in Zucker lean, obese, and obese diabetic (type 2) rats. We compared the $\beta$ adrenoceptor signaling pathway in Zucker lean, Zucker obese, and Zucker obese diabetic rats, the reliable rat models of metabolic syndrome. The effects of $\beta$-adrenoceptor stimulation were investigated in vivo with transthoracic echocardiography and in vitro in isolated left ventricular papillary muscles in Zucker lean (control), obese, and obese diabetic rats. The expressions of $\beta_{1^{-}}, \beta_{2^{-}}, \beta_{3}$-adrenoceptors and multidrug resistance-associated protein 4 in left ventricular muscles were detected by Western Blotting. The plasma concentrations of triglyceride, cholesterol, leptin, adiponectin, and C-peptide were measured using automated enzymatic method, or quantitative enzyme-linked immunosorbent assay (ELISA) kits. Data are presented as mean \pm SD. Hyperlipidemia, high leptin, and C-peptide concentrations were observed in obese and obese diabetic strains, whereas hyperglycemia occurred only in the diabetic strain. No significant difference among strains was observed in echocardiography in 
vivo. In vitro, the positive inotropic effect of isoproterenol was slightly reduced in obese rat $(183 \% \pm 11 \%$ of baseline, $P=0.003 ; \mathrm{n}=7)$ and markedly reduced in obese diabetic rats $(137 \%$ $\pm 18 \%$ of baseline, $P<0.001 ; \mathrm{n}=10)$ when compared with control rats $(210 \% \pm 17 \%$ of baseline; $n=9)$. $\beta_{1}$-adrenoceptor protein expression were down-regulated in obese $(-41 \%$, $P=0.02)$ and diabetic rats $(-54 \%, P=0.003)$ when compared with control rats, and a similar decrease was observed in $\beta_{2}$-adrenoceptors protein expression. But $\beta_{3}$-adrenoceptor and multidrug resistance-associated protein 4 expressions remained unchanged. The lusitropic effect of isoproterenol was not modified among strains. The positive inotropic effect of forskolin slightly decreased in obese rats $(148 \% \pm 25 \%$ of baseline value, $P=0.005)$ and markedly decreased in obese diabetic rats $(118 \% \pm 12 \%$ of baseline value, $P<0.001)$ when compared with control rats $\left(181 \% \pm 23 \%\right.$ of baseline value). Upon stimulation of $33^{\prime}, 5^{\prime}$-cyclic adenosine monophosphate by dibutyryl cAMP, the positive inotropic effect in control rats $(185 \% \pm 19 \%$ of baseline value) was significantly higher than that in obese rats or obese diabetic rats $(158 \% \pm 36 \%, 155 \% \pm 27 \%$ of baseline value; $P=0.043, P=0.031$, respectively). Direct stimulation of adenylate cyclase with forskolin and administration of 3 ',5'-cyclic adenosine monophosphate suggests that subtle impairments also occur upstream and downstream of the adenylate cyclase level of the $\beta$-adrenergic pathway in obese and diabetic rats, besides the down-regulation of $\beta_{1^{-}}$and $\beta_{2}$-adrenoceptor. In conclusion, the positive inotropic effect of $\beta$-adrenoceptor stimulation is slightly decreased in Zucker obese rats and is more markedly decreased in Zucker obese diabetic rats. These decreases are mainly related to $\beta_{1}$ - and $\beta_{2}$-adrenoceptors down-regulation.

Key words $\beta$-adrenoceptors; cardiac muscle; catecholamines; obesity; diabetes mellitus 


\section{中文摘要}

在许多心血管疾病中, 包括从高血压、心肌病在内的各种慢性病到最终的充血性心 力衰竭, 交感神经系统进行性地被激活已经得到确认。[82,156,412-414] 交感神经系统的高 活性可以改变 $\beta$-肾上腺素受体的数量、功能以及其下游机制。尽管目前尚无简单统一 的机制可以解释这些不同的发现，钲离子活动的异常却已经被认为是左心室收缩功能 不全的一大基础原因 ${ }^{[82]}$ 。细胞内钙离子的水平直接影响着肌动蛋白与肌球蛋白的相互 作用, 从而影响着心肌收缩性。 $\beta$ - 肾上腺素能刺激可以通过刺激生成环磷酸腺苷

（cAMP），并激活蛋白激酶 A（PKA），介导产生正性肌力/松弛力效应。随后，一 系列目标蛋白（钲离子通道，兰尼碱受体，肌浆网钻泵，以及肌钻蛋白）紧接着被磷 酸化后, 钲离子瞬变得以增强。在病理生理状态下, $\beta$-肾上腺素能功能不全是心功能 减低的一类常见机制。因此, 我们分别针对衰老和代谢综合征相关心肌病, 进行了有 关 $\beta$-肾上腺素受体刺激通路的两项研究。

第一项研究针对衰老大鼠进行。在衰老心脏中, 存在舒张功能不全和对 $\beta$-肾上腺 素能刺激的效应下降。这些与 $\beta_{1}$-和 $\beta_{2}$-肾上腺素受体的下调, 以及 $\beta 3$-肾上腺素受体的 上调有关。[73,415,416] 这些改变要么减少 cAMP 的产生, 要么促进 cAMP 的水解。同时 还有其他调节 cAMP 的相关机制也在心脏中起到作用。多药耐药相关蛋白 4（MRP4） 在心肌细胞内 $\mathrm{cAMP}$ 的调节和 $\beta$-肾上腺素能刺激效应方面起到了重要作用。但是 MRP4 在衰老心脏中的作用尚无相关研究。因此, 我们进行了本研究, 目的是观察衰 老心脏中 MRP4 的表达和其对 $\beta$-肾上腺素能功能不全的影响。在年轻大鼠 (3 月龄) 和衰老大鼠 (24 月龄) 中, 心脏对异丙肾上腺素刺激后的 $\beta$-肾上腺素能效应通过在体 (超声心动图) 和离体（离体心肌细胞通过 Ionoptix $囚$ 观察肌节的缩短和钻瞬变）。其 效应的研究将在是否使用 MK571（一种特异性 MRP4 抑制剂）进行预处理之间进行对 比。左心室组织的 MRP4 蛋白表达将通过免疫印迹法进行测定。实验结果显示, 我们 确认了 MRP4 蛋白的过度表达是导致衰老心脏中 $\beta$-肾上腺素能刺激后正性肌力反应下 降的一个原因。第二项研究针对代谢综合征大鼠进行。尽管由于代谢综合征存在的交 感神经活动性增加, 会对 $\beta$-肾上腺素受体产生慢性刺激, 但是, $\beta$-肾上腺素能受体信 号传导通路在这种情况下的研究仍较少。因此, 我们研究了 Zucker 瘦, 肥胖和肥胖糖 尿病（2 型糖尿病）大鼠的 $\beta$-肾上腺素能受体信号传导通路的变化。利用 Zucker 瘦, 肥胖和肥胖糖尿病 (2 型糖尿病) 大鼠, $\beta$-肾上腺素能的反应性得以研究。我们研究在 体超声心动图下 $\beta$-肾上腺素受体的刺激效应, 还进行了离体左心室乳头肌的反应性研 究, 并对 $\beta_{1^{-}}, \beta_{2}$-和 $\beta_{3}$-肾上腺素受体和多药耐药相关蛋白 4 进行免疫印迹定量研究。 同时, 利用自动酶标法或定量酶联免疫吸附测定试剂盒, 我们测定血浆中甘油三酯、 胆固醇、瘦素、脂联素以及 C-肽的浓度。数据以平均值土标准差表达。在 Zucker 肥胖 和肥胖糖尿病大鼠中, 我们观察到高脂血症, 高瘦素和高 C-肽浓度, 但是, 只有肥胖 糖尿病大鼠可以观察到高血糖。在体实验中, 各种系大鼠的超声心动图表现无显著性 差异。离体实验中, 与对照组（基线的 $210 \% \pm 17 \%, \mathrm{n}=9$ ）相比, 异丙肾上腺素的正 性肌力作用在肥胖大鼠中略有减少（基线的 $183 \% \pm 11 \%, \mathrm{P}=0.003 ; \mathrm{n}=7$ ），而在肥 胖糖尿病大鼠中则显著降低（基线的 $137 \% \pm 18 \%, \mathrm{P}<0.001 ; \mathrm{n}=10$ ）。 $\beta_{1}$-肾上腺素受 体蛋白表达在肥胖大鼠中减少 $(-41 \%, \mathrm{P}=0.02)$, 在肥胖糖尿病大鼠中也显著减少（$54 \%, P=0.003$ ）； $\beta_{2}$-肾上腺素受体蛋白具有与 $\beta_{1}$-肾上腺素受体相似的表达减少; 但 $\beta 3$-肾上腺素受体和多药耐药相关蛋白 4 的表达保持不变。各种系动物在异丙肾上腺素 诱导中的松弛性影响均无显著性差异。与对照组（基线的 $181 \% \pm 23 \%$ ）相比， Forskolin 刺激产生的正性肌力作用在肥胖大鼠中略有减少（基线的 $148 \% \pm 25 \%, \mathrm{P}$ 
$=0.005$ ），而在肥胖糖尿病大鼠中出现显著减少（基线的 $118 \% \pm 12 \%, \mathrm{P}<0.001$ ）。当 直接用 $3^{\prime}, 5^{\prime}$-环化腺苷酸类似物刺激时，在对照组大鼠中产生的正性肌力效应（基线的 $185 \% \pm 19 \%$ ）显著高于肥胖大鼠或肥胖糖尿病大鼠（基线的 $158 \% \pm 36 \%, 155 \% \pm 27 \%$; 分别 $\mathrm{P}=0.043, \mathrm{P}=0.031$ ）。用 Forskolin 刺激腺苷酸环化酶, 或用 $3^{\prime}, 5^{\prime}$-环化腺苷酸类似 物直接刺激的结果显示: 除了存在 $\beta_{1}$-和 $\beta_{2}$ - 肾上腺素受体的下调, 肥胖和肥胖糖尿病 大鼠的 $\beta$-肾上腺素能通路中, 腺苷酸环化酶水平的上游和下游, 均有可能存在轻微的 受损。Zucker 肥胖大鼠中， $\beta$-肾上腺素受体激动的正性肌力效应略有下降，而 Zucker 肥胖糖尿病大鼠的反应性则更为显著地下降。这些效应主要与 $\beta_{1}$-和 $\beta_{2}$-肾上腺素受体 的下调有关。

关键词： $\beta$-肾上腺素受体；心肌；儿茶酚胺；肥胖；糖尿病 